University of Rhode Island

DigitalCommons@URI

Open Access Master's Theses

2020

\title{
STUDY OF THE AUTOMATION OF DISASSEMBLY OF ELECTRIC VEHICLE BATTERIES
}

Jan Friedrich Hellmuth

University of Rhode Island, fritz.hellmuth@arcor.de

Follow this and additional works at: https://digitalcommons.uri.edu/theses

\section{Recommended Citation}

Hellmuth, Jan Friedrich, "STUDY OF THE AUTOMATION OF DISASSEMBLY OF ELECTRIC VEHICLE BATTERIES" (2020). Open Access Master's Theses. Paper 1869.

https://digitalcommons.uri.edu/theses/1869

This Thesis is brought to you for free and open access by DigitalCommons@URI. It has been accepted for inclusion in Open Access Master's Theses by an authorized administrator of DigitalCommons@URI. For more information, please contact digitalcommons@etal.uri.edu. 


\section{STUDY OF THE AUTOMATION OF DISASSEMBLY OF \\ ELECTRIC VEHICLE BATTERIES}

BY

JAN FRIEDRICH HELLMUTH

A THESIS SUBMITTED IN PARTIAL FULFILLMENT OF THE

REQUIREMENTS FOR THE DEGREE OF

MASTER OF SCIENCE

IN

MECHANICAL ENGINEERING AND APPLIED MECHANICS

UNIVERSITY OF RHODE ISLAND

2020 
MASTER OF SIENCE THESIS

OF

JAN FRIEDRICH HELLMUTH

\section{APPROVED:}

Thesis Committee:

Major Professor Musa Jouaneh

Chengzhi Yuan

Jason Dahl

Nasser H. Zawia

DEAN OF THE GRADUATE SCHOOL

UNIVERSITY OF RHODE ISLAND

2020 


\section{ABSTRACT}

Electric vehicles offer an environment friendly solution to mobility. An assessment of automation potentials in the recycling of electric vehicle (EV) batteries and a simulation of two different disassembly cell layouts was conducted in this thesis. This study was broken up into three distinct parts. First a literature review is presented for examining recent developments and challenges in the disassembly of electric vehicle batteries. Because of the large variety in the designs of EV batteries, human-robot collaboration was suggested. Based on the review, an assessment of automation potentials was conducted using as an example the 2017 Chevrolet Bolt battery. A disassembly graph was developed that shows all constraints of the parts and fastener and a disassembly sequence with 46 disassembly steps was also developed. For assessing single disassembly steps in terms of economic feasibility and technical possibilities in automation, a criteria catalogue was developed and applied on a large battery electric vehicle and a small hybrid electric vehicle. The results were compared to similar assessments and a comparison of the different types of EV batteries towards disassembly was conducted. For large battery electric vehicles automation of disassembly operations is more feasible but also technically more challenging. In the third part, a simulation disassembly layout was created, that compares a layout with a Cartesian gantry robot with a layout that used two collaborative robots. It was shown that the collaborative robots that were proposed for the disassembly of hybrid vehicle batteries face difficulties in disassembly of large battery electric vehicles due to the large size and heavy parts. The comparison of both layouts favors the use of a Cartesian gantry robot because the disassembly is faster and also the disassembly steps that include large and heavy parts can also be performed. 


\section{ACKNOWLEDGMENTS}

I would like to thank my major professor, Dr. Musa Jouaneh for supervising my research work and for his continuous support and guidance during the writing of this Thesis, and in general as a University of Rhode Island graduate student. I really appreciate the knowledge and understanding he gave me in the topics of robotics and disassembly. Also, I would like thank Dr. Nicholas DiFilippo for discussing parts of the research with me and giving advice, especially I appreciate our corporation on the development of the disassembly graph. At this point I would like to thank the committee members, Dr. Chengzhi Yuan and Dr. Jason Dahl as well as the thesis defense chair Dr. Sigrid Berka.

I would also like to thank my family for the steady long-distance mental support during my year at the University of Rhode Island. Additionally, I would like to thank my girlfriend Aleksandra for steadily supporting me doing my research, motivating me and listening to my ideas and discussing approaches. 


\section{Table of Contents}

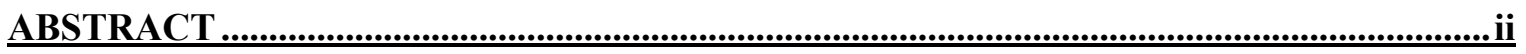

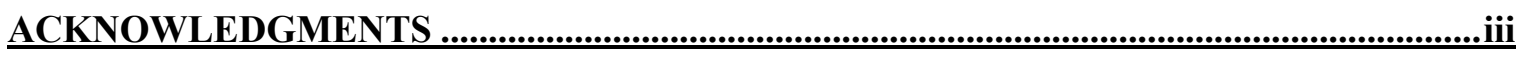

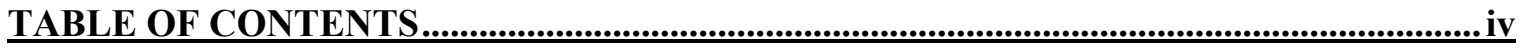

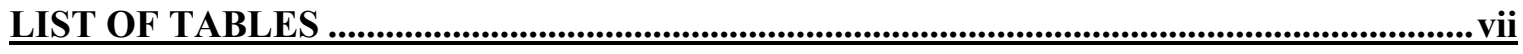

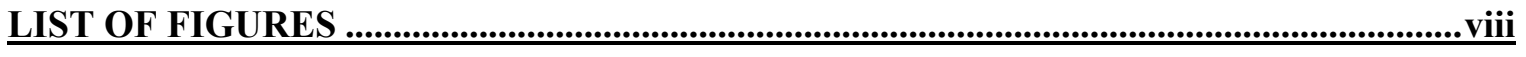

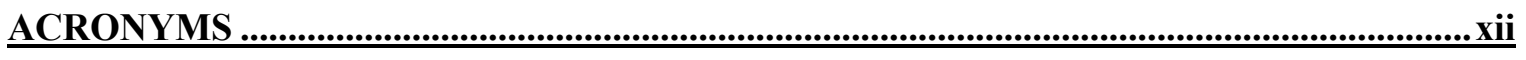

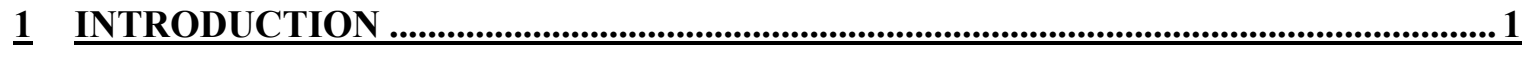

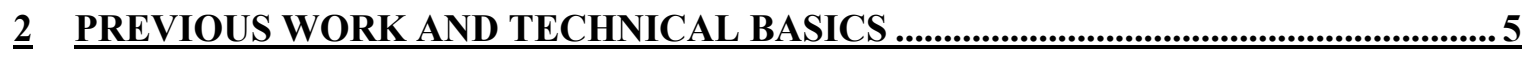

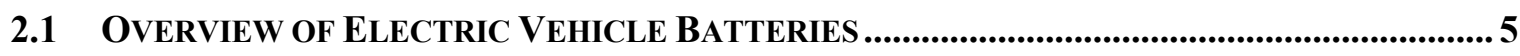

2.1.1 DESIGNS OF BATTERIES AND RECYCLING APPROACHES ....................................... 6

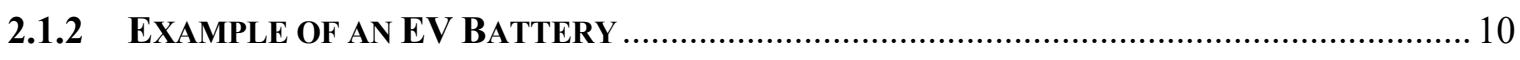

2.2 DISASSEMBLY STRATEGIES ON E-WASTE AND EV BATTERIES......................................... 12

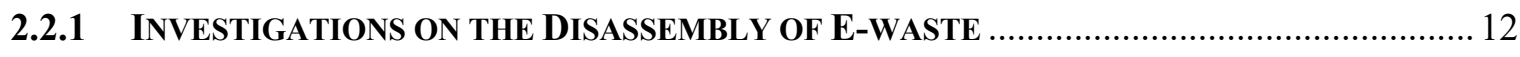

2.2.2 STRUCTURE ANALYSIS OF EV BATTERIES AND STEP ASSESSMENT IN DiSASSEMBLY. 17

2.2.3 DISASSEMBLY CONCEPTS FOR ELECTRIC VEHICLE BATTERIES............................... 21

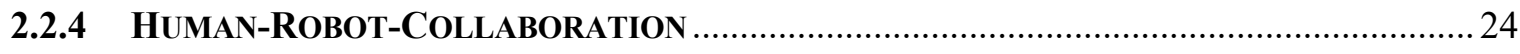

2.3 PROPOSED CONCEPTS FOR DIFFERENT DISASSEMBLY STEPS ............................................27

2.3.1 DETECTION OF FASTENERS AND PARTS BY VISION SYSTEMS …..............................2 


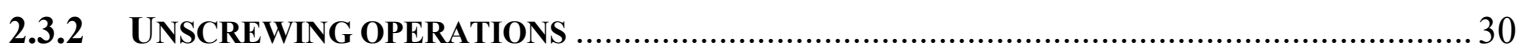

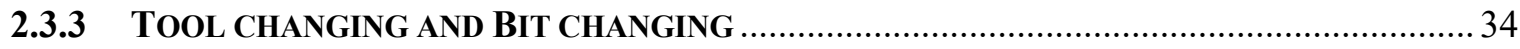

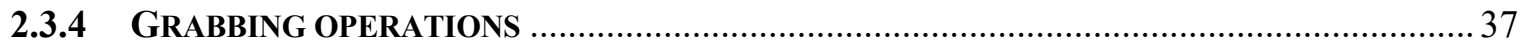

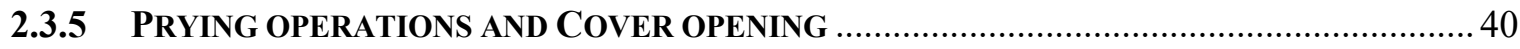

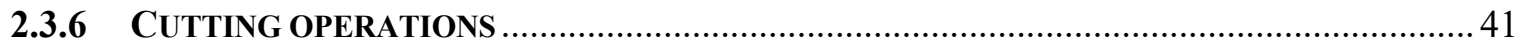

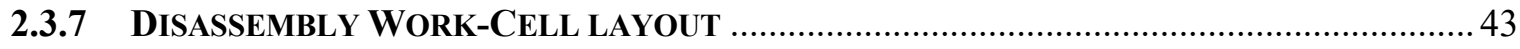

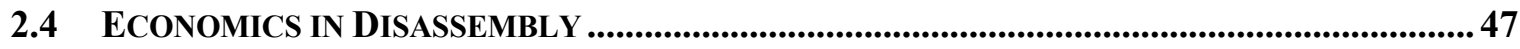

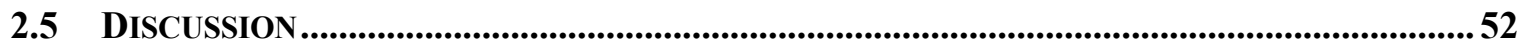

$\underline{3}$ ANALYSIS AND ASSESSMENT OF EV BATTERY RECYCLING .........................58

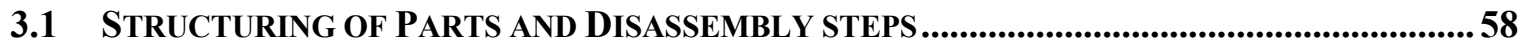

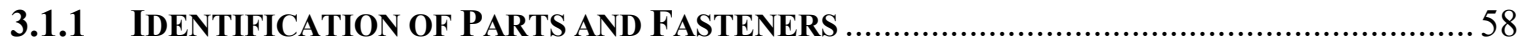

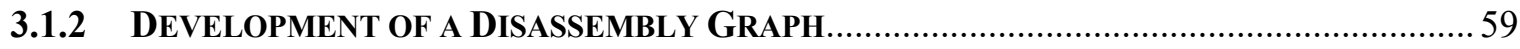

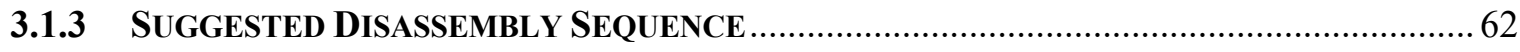

3.2 ASSESSMENT OF THE AUTOMATION POTENTIAL OF DISASSEMBLY STEPS ...................... 67

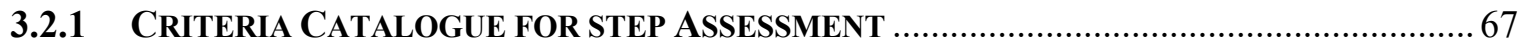

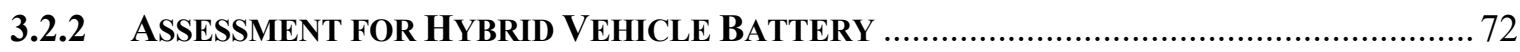

3.2.3 ASSESSMENT FOR BATTERY ELECTRIC VEHICLE BATTERY .................................. 75

3.2.4 COMPARISON OF THE ASSESSMENT OF THE DIFFERENT BATTERY TYPES................... 77

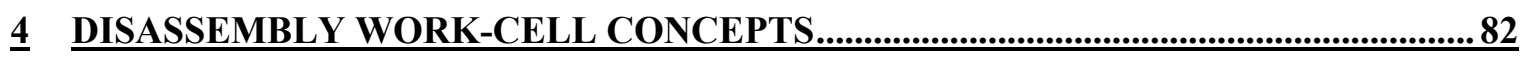

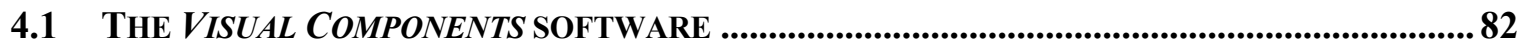

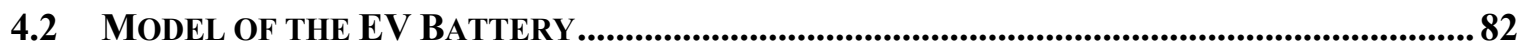

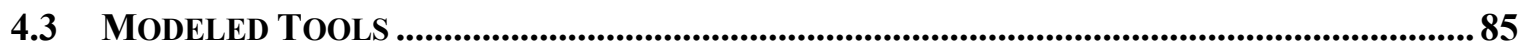

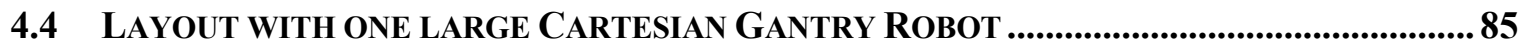




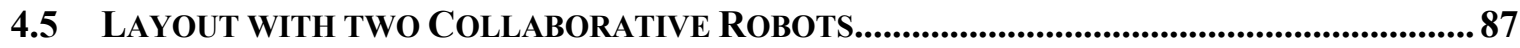

4.6 DISASSEMBLY SEQUENCE FOR THE MODELED BEV BATTERY AND COMPARISON OF THE

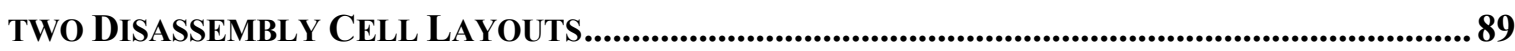

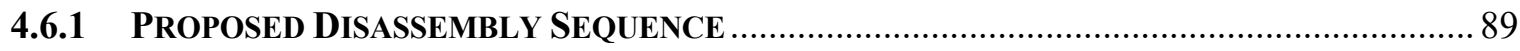

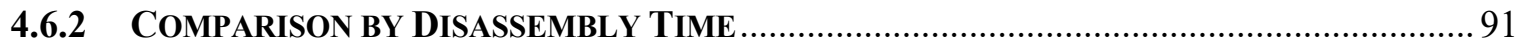

4.6.3 CALCULATION OF DISASSEMbLY TIMES FOR EXAMPLE BEV BATTERY .................... 92

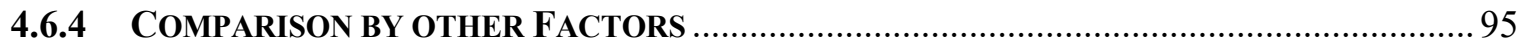

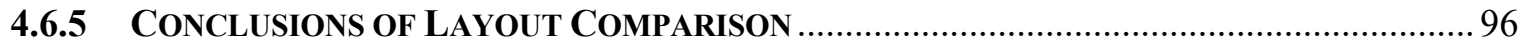

4.7 FURTHER SUGGESTIONS FOR EV BATTERY DISASSEMBLY.............................................98

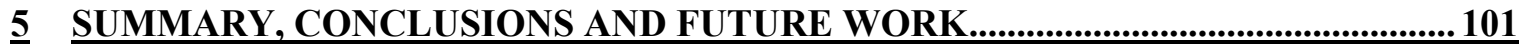

6

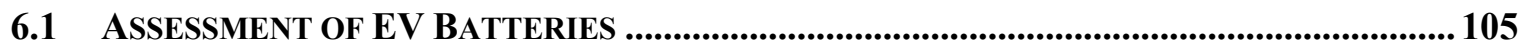

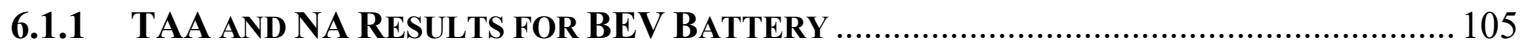

6.1.2 DETAILED ASSESSMENTS OF EACH DISASSEMBLY STEP FOR BEV BATTERY ….......... 108

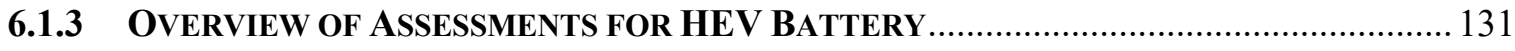

6.1.4 DETAILED ASSESSMENTS OF EACH DISASSEMBLY STEP FOR HEV BATTERY …......... 132

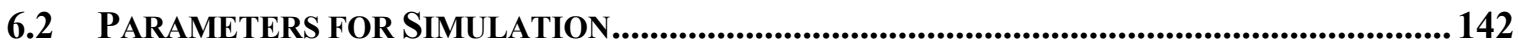

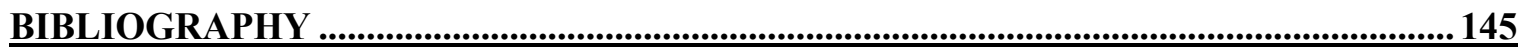




\section{List of Tables}

Table 1: Description and image of a fastener.

Table 2: Examples of disassembly step descriptions

Table 3: Assessment criteria 68

Table 4: Criteria scorings on part weights and ergonomics (NA4) 69

Table 5: Criteria scorings on the priority for disassembly (NA5) 69

Table 6: Criteria scorings for the complexity of robotic motion (TAA1) 70

Table 7: Criteria scorings for the access (TAA2) 70

Table 8: Criteria scorings for the detection (TAA3) 71

Table 9: Criteria scorings for the automation potentials of the robotic end-effector (TAA4)

Table 10: Assessment on the Unscrewing of covers for the Audi Q5 hybrid vehicle battery .72

Table 11: Modeled Parts and Fasteners for Disassembly Simulation 83

Table 12: Disassembly Sequences for modeled layouts .90

Table 13: Comparison of two disassembly cell layouts .97 


\section{List of Figures}

Figure 1: Structure of an EV battery on the example of the Audi A3 Sportback e-tron [9] 7

Figure 2: Comparison of different EV battery designs [11] ...................................... 8

Figure 3: 2017 Chevrolet Bolt battery with removed cover, adapted from [21] ............... 12

Figure 4: Example of a disassembly constraint graph for crystal liquid displays [23] ...... 14

Figure 5: Graph model of the environment [46] .......................................................... 15

Figure 6: Design and parts of the Audi Q5 hybrid battery [59] ..................................... 18

Figure 7: Results of the portfolio analysis plotted in a scatter diagram [60] f...................22

Figure 8: Classification of hybrid assembly, adapted from [72] ..................................25

Figure 9: View of the Vision System for laptop recycling [52] .....................................2 28

Figure 10: Robotic end-effector with automated screwdriver [6].................................... 31

Figure 11: Sketch (a) and prototype (b) of an automated screwdriver [52]........................32

Figure 12: Desired final position of simulated robot for unscrewing with RL algorithm

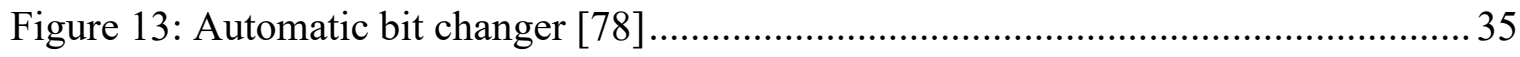

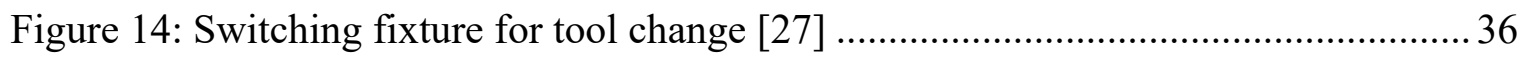

Figure 15: Three-finger gripper for disassembly [82] [28] ............................................ 37

Figure 16: Two-finger gripper for disassembly [29] [28] ............................................... 38 
Figure 17: Sketch of a gripper boring into disassembly parts [30]

Figure 18: Gripper system for EV battery cell extraction [63]

Figure 19: Prototype design of cover opening tool [31]

Figure 20: Robot for the disassembly of mobile phones with included cutting tool [32].. 42

Figure 21: Disassembly work cell for EV battery disassembly, adapted from [10] .44

Figure 22: Working table with robot for EV battery disassembly ,adapted from [10] ..... 44

Figure 23: Layout of a disassembly cell for TVs and monitors [33] 46

Figure 24: Software structure of a disassembly [33] 47

Figure 25: Model for assessing the economics of disassembly [2]

Figure 26: Disassembly graph (page 1) 60

Figure 27: Disassembly graph (page 2) 61

Figure 28: Disassembly graph (page 3) 62

Figure 29: Disassembly step D1, the Top Cover [21] 64

Figure 30: Disassembly step D6, the four covers for the busbars in the front [21] 65

Figure 31: Disassembly step D12, the Big Nuts for Coolant Hoses [21] 65

Figure 32: Example for screws in disassembly step D19 [21] 66

Figure 33: Disassembly step D39, the lifting of four battery modules [21] 66

Figure 34: Assessments of disassembly steps for Audi Q5 hybrid vehicle battery 74 
Figure 35: Assessment of disassembly steps for 2017 Chevrolet Bolt battery 76

Figure 36: Assessment of disassembly steps for several BEV and HEV/PHEV batteries, adapted from [60]

Figure 37: Modeled BEV Battery, a) Complete battery, b) Battery with removed Top

Cover 84

Figure 38: Layout with one large cartesian gantry robot 86

Figure 39: Layout with two collaborative robots 88

Figure 40: Collaborative robots layout: a) Changing of Unscrewing Tool, b) Unscrewing of Bolts for Brackets, c) Unscrewing of Bolts around Top Cover, d) Linear slide with a collaborative UR10e robot on it, e) Disassembly of the Top Cover by the human worker

Figure 41: Examples of disassembly steps: a) Unscrewing of Bolts around the Top Cover, b) Grabbing of the Top Cover by the Suction Gripper, c) Delivering the Top Cover to the Conveyor, d) The human worker disassembling the Cable .90

Figure 42: Disassembly time for disassembly with Gantry robot for modeled Battery..... 91

Figure 43: Disassembly time for disassembly of modeled BEV battery with two collaborative UR10e robots. 92

Figure 44: Calculated disassembly time for the 46 disassembly steps of the 2017 Chevrolet Bolt Battery with a gantry robot 94

Figure 45: Calculated disassembly time for the 46 disassembly steps of the 2017 Chevrolet Bolt Battery with two collaborative robots 95 
Figure 46: Extended Gantry robot layout with a suggestion for the further treatment of the disassembled parts. 100

Figure 47:a) The sorting robot, b) Sorting Brackets on the Conveyor for metal trash, c) Collaborative workstation for Battery Module/Section disassembly d) Metal trash collection 100 


\section{Acronyms}

\begin{tabular}{|c|c|}
\hline BECM & Battery energy control module \\
\hline $\mathrm{BEV}$ & Battery electric vehicle \\
\hline $\mathrm{BMC}$ & Battery management controller \\
\hline BMS & Battery management system \\
\hline $\mathrm{DC}$ & Direct Current \\
\hline DfAD & Design for assembly and disassembly \\
\hline DFD & Design for disassembly \\
\hline EOL & End-of-life \\
\hline $\mathrm{EV}$ & Electric vehicle \\
\hline FSR & Force resisting sensor \\
\hline $\mathrm{HEV}$ & Hybrid electric vehicle \\
\hline HRI & Human-robot interaction \\
\hline $\mathrm{HV}$ & High voltage \\
\hline LWR & Light weight robot \\
\hline MTM & Methods-Time Measurements \\
\hline NA & Necessity to automate \\
\hline PHEV & Plug- in hybrid electric vehicle \\
\hline QR & Quick response \\
\hline RFID & Radio frequency identification \\
\hline RL & Reinforcement Learning \\
\hline TAA & Technical ability to automate \\
\hline UR & Universal Robots \\
\hline
\end{tabular}




\section{Introduction}

Climate change is one of the biggest threats to the environment and humanity today, with mobility being one of the largest producers of greenhouse gases [1]. In order to reduce the emissions of greenhouse gases many countries worldwide promote the spread of electric vehicles (EV). In comparison to conventional cars with an internal combustion engine, EVs use simpler electrical motors instead of large engines with many individual parts. Electric vehicles need a large EV battery for carrying the energy. Since range is an important indicator for the competitiveness of EVs, batteries are large and heavy for providing that range. Also, EV batteries contain expensive materials such as lithium or cobalt that contribute to a large amount of the production costs of an EV [2]. The numbers of sales of EVs rise constantly and using a lifespan of 10-15 years the treatment of disposed EV batteries is increasingly an important field of research.

The worst method for treating disposed EV batteries would be landfill because expensive materials are wasted [3] and it has a negative environmental impact because of the disposed batteries still contain hazardous materials [4]. Another current method is manual disassembly and extraction of the valuable parts. After that the battery cells are treated pyro metallurgically [5]. For later treatment the battery cells need to get extracted. The current manual process is very expensive. High labor costs and workers' protection from high-voltage and chemical hazards drive the costs. Many of the disassembly steps in EV battery disassembly are also very repetitive. Such steps include unscrewing or

grabbing operations. For such disassembly operations, automation is necessary for reducing costs and making EV battery recycling more attractive. 


\section{Overview of Thesis}

In addition to the Introduction Chapter, this thesis has four additional chapters.

In Chapter 2, a review of some design approaches will be provided and the main components of an EV battery will be explained using an example. A short summary of the recycling and metal recovering techniques following the disassembly of EV batteries will also be given.

There are many different designs for EV batteries. That leads to a higher complexity in EV battery disassembly [6]. The field of automated EV battery recycling is relatively new. However, there was a lot of research on the disassembly of electronic equipment such as personal computers or televisions. A summary of those studies in the next chapter will show achievements in disassembly planning and different ideas for optimizations of disassembly processes. EV batteries are large products. It is necessary to analyze the product structure in order to plan an efficient disassembly sequence. However, some of the disassembly steps would be very difficult for the current state of the art in robotics. Additionally, due to the large design's varieties, products at the end of their lifespan could also be damaged or be in a dirty environment in the disassembly area that could impede the robotics sensors. This suggests a division of tasks between human workers and robots. For every disassembly step it needs to get decided if it should be done automatically or manually. An assessment approach will be presented in this thesis to help with this decision. With that knowledge, techniques for the disassembly steps that strongly need to be automated can be developed. While current studies on EV battery recycling took a closer look on smaller hybrid vehicle batteries this study aims to create ideas for the disassembly of large EV batteries. The division of tasks makes human-robot- 
collaboration necessary. A short explanation of human-robot-collaboration will be given and the main difficulties and studies on that topic will be discussed.

There are different operations, that a partly automated system for EV battery disassembly needs to perform. Those include a vision system that could be described as an eye that identifies parts and fasteners and their locations and supervises the work of the robot. For fasteners, automated unscrewing techniques are also needed. Since there are different types of fasteners in such a complex product, also an automated tool- or bitchanging system is necessary. Furthermore, a grabbing tool is needed to collect disconnected parts and a prying tool to flip covers, while a cutting tool will be needed for cutting cables or hoses. Current investigations on such single robotic skills for disassembly will be summarized. Also, some publications on disassembly work-cell design in general and the requirements for a work-cell for EV battery disassembly will be discussed. As mentioned before, EV battery disassembly must be economically feasible and an attractive business. Publications on the economics and prediction on the number of recyclable EV batteries, costs and revenues will be summarized.

In Chapter 3, an analysis of the structure of an example of an EV battery will be performed. The presented disassembly graph includes all dependencies along the parts and fasteners of the EV battery. All connections are included. Based on that, a disassembly sequence can be developed that includes several disassembly steps with a certain repetition of one or a few similar operations. For each disassembly step detailed information are documented and a first approximation of the automation potential is given. Based on that a criteria catalogue was developed for determining technical and economical automation potentials for each disassembly step. The results were compared 
with a similar study and differences in the assessment of disassembly steps of small hybrid vehicle Batteries and large electric vehicle batteries were discussed.

In Chapter 4 a simulation on the disassembly of a simplified battery will be performed. Therefore, at first a model of the 2017 Chevrolet Bolt battery will be presented, and it will be discussed, which parts should be modeled for assessing the most important disassembly steps. After that, one layout with a one gantry robot and one layout with two collaborative robots will be described. A disassembly sequence for disassembling of the simple modeled battery will be presented. Both layouts will be compared by disassembly time, costs, ability to perform all operations and suitability for human-robot collaboration. Based on the observed disassembly times a calculation scheme for predicting the disassembly time for real EV batteries will be presented and the results for the 2017 Chevrolet Bolt battery with the both layouts will be discussed. Further ideas for a disassembly work cell layout and the processing of the disassembled parts and fasteners will be presented.

A summary, the conclusions of this study and an outlook for future research will be given in Chapter 5 


\section{Previous Work and Technical Basics}

The discussed literature for this thesis clusters into four main areas. The first section gives an overview of electric vehicle batteries their design and some recent studies on the recycling of those. In addition, one example is described in detail. The second area reviews work on the recycling of electronic waste in general such as personal computers. The third area reviews concepts for single disassembly operations or necessary functions in the disassembly process. The last area reviews economics in the disassembly of EV batteries will be discussed. Those discussions include market predictions and models for determining if the disassembly of EV batteries is economically feasible, even if it would be legally required.

\subsection{Overview of Electric Vehicle Batteries}

There are three types of electric vehicles that use different types and sizes of EV batteries. Battery electric vehicles (BEV) only use electric energy and do not have an internal combustion engine or a fuel tank. Examples of BEVs are the Chevrolet Bolt or

Tesla Model S. BEV batteries are usually the largest and heaviest. Hybrid electric vehicles (HEV) use both, an internal combustion engine and an electric propulsion system. The main goal is achieving better fuel economy. An example would be the Toyota Prius. HEV batteries are much smaller, because only a very short only electric driving range is provided. Plug-in hybrid electric vehicles (PHEV) use an internal combustion engine, but also use an electric motor and a battery that can be plugged to external sources of electricity. Examples for PHEVs are the Chevrolet Volt, or Porsche Panamera 4 EHybrid. The goal is to provide a certain range of only electric driving and higher driving 
performance and efficiency due to this combination. For example, with an PHEV it is possible to drive distances inside cities only electric and use the internal combustion engine for long-distance driving. The PHEV batteries have sizes and weights in between of BEV and HEV.

\subsubsection{Designs of Batteries and Recycling Approaches}

According to Ketterer [7] Lithium-ion technology is the most used in EV batteries. This is due to high energy density and power compared to other battery technologies. Since there is a need for high power and a lot of energy for EV batteries many single cells are needed for one EV battery. Therefore, cells are bracketed together in modules. It is possible to interconnect the cells inside the modules in series or parallel circuits. As a next step several modules are combined and interconnected to form the EV battery. There are three main types of battery cells, that are used for EVs: Prismatic cells, cylindrical cells and pouch cells. While cylindrical cells are cheaper to produce and mechanically more stable, pouch cells are not as heavy as the other types.

Weyrich and Natkunarajah [8] described the components in an EV battery (an example is shown in Figure 1). The components include a certain number of modules, there are clamp elements and different kinds of cables. Furthermore, every EV has a battery management controller (BMC) that is sometimes also called battery management systems (BMS) or battery management unit (BMU). Most EV batteries have also a cooling system and an insulation. There is a housing that covers around the battery, that usually consists of two parts, a lower tray and an upper cover. The modules contain the battery cells, a cell management controller and cables. 


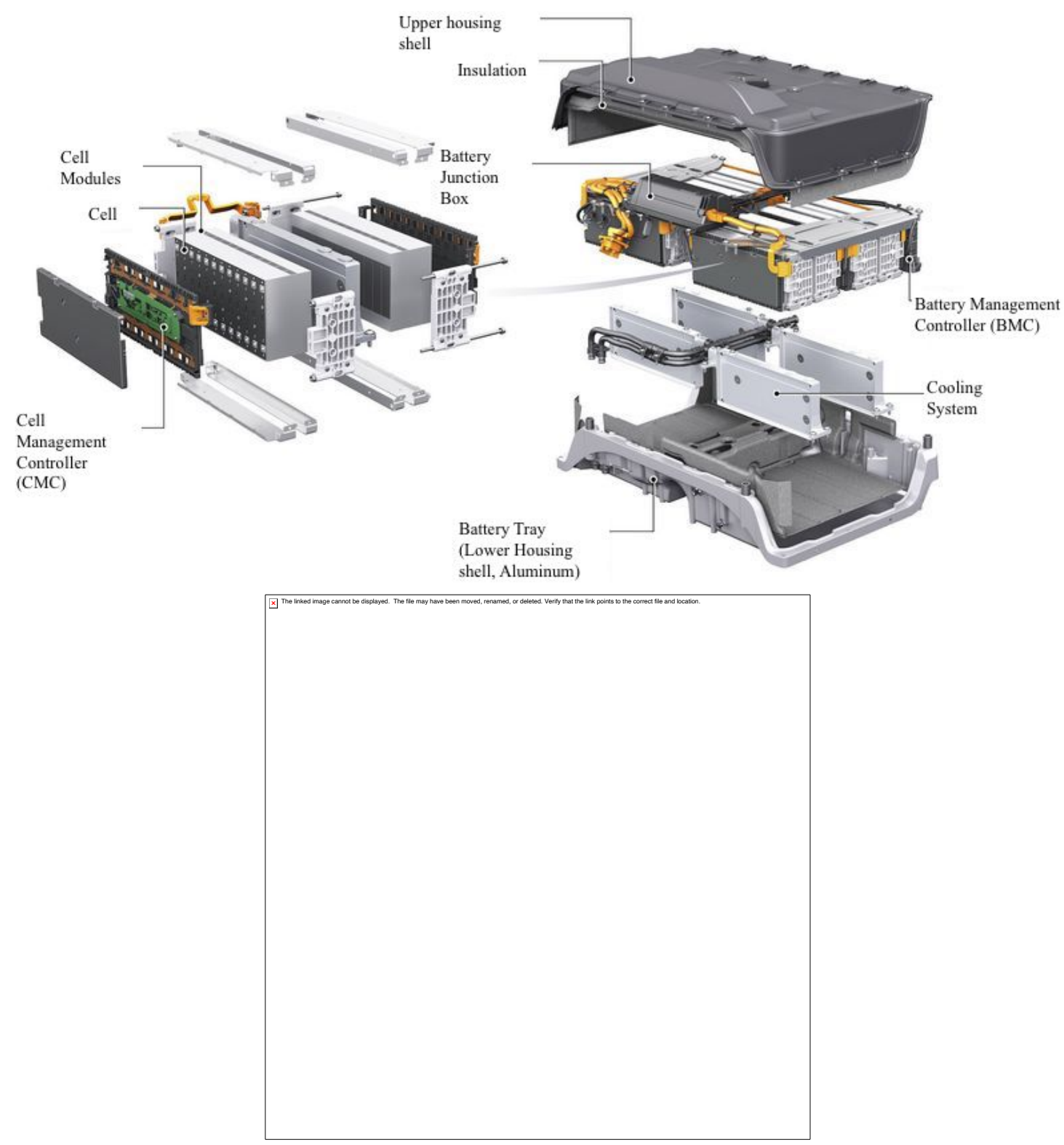

Figure 1: Structure of an EV battery on the example of the Audi A3 Sportback e-tron [9]

According to Wegener [10] the design of EV batteries is missing common standards as there is a very high diversity of variants. One reason is that there are different types of EVs such as PHEVs, BEVs and HEVs and those different types require different battery designs. There is also a large variety of battery manufacturers (e.g. LG Chem, A123 Systems and Envision AESC). Because of this, there are many varying designs, sizes, weights, and structures of EV batteries. 
Harper et al. [11] compared different designs of EV batteries. It was mentioned, that the Tesla Model $S$ uses cylindrical cells. There is a medium number of modules (16) in one battery pack, but there are many (444) small battery cells in one module. Contrary to that, the Nissan Leaf uses an EV battery design with many (48) small modules with just 4 large cells in each. The $B M W i 3$ battery is composed of just 8 battery modules with 12 large cells in each. In Figure 2 those three different EV battery designs with different types of cells are compared. It was mentioned that the shares of the expensive metal cobalt vary among the three batteries. The cells of the Nissan Leaf contain relatively low levels of cobalt compared to those of the Tesla Model S or the BMW i3.

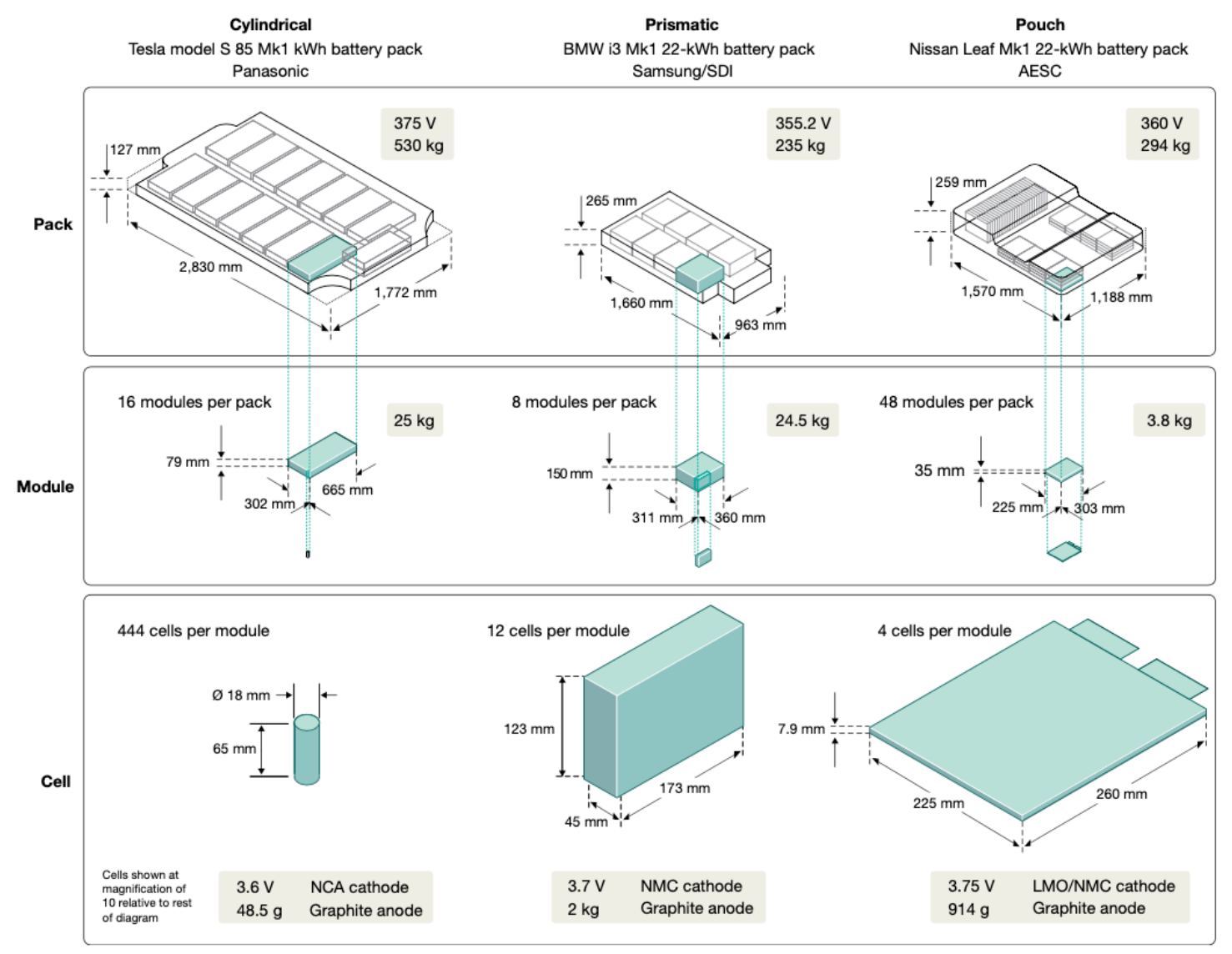

Figure 2: Comparison of different EV battery designs [11] 
Tornow et al. [12] described, that about two thirds of the costs for an EV battery originate from the cells raw materials and their production. Furthermore, EV batteries are made of many multi-material parts. Those parts create further challenges for disassembly. Another challenge to disassembly could be the difficulty of removing fasteners, for example fasteners that faced corrosion, or fasteners hidden below other parts. The EV battery in general is described as having three different hierarchy levels: Battery cell level, battery module level and battery system level. The level structure helps for better labor division in battery production.

Elwert et al. [13] summarized the legislation of EV battery recycling in China, the USA and Europe and gave predictions for the amount of recyclable batteries for the years until 2025. A further description of the recycling process was given. Also, Choi and Rhee [14] compared practice of EV battery recycling in Korea with other countries such as China or European countries. Liu et al. [15] assessed the situation of EV battery recycling in China. Different levels of reusing that could be established before disassembly were described.

Gaines [16] described the whole recycling process of EV batteries and different recycling methods for the battery cells after disassembly. The recycling methods for the battery cells were compared [17]. Also, the possibility of a second use was described. It was stated, that the recycling of EV batteries with a low cobalt content would only be economically feasible if there will be further developments in direct recycling and recycling-friendly design. Ahmadi et al. [18] described the "second life" use of EV batteries in details. These include the usage as stationary energy storages for a smart electrical grid. Mossali et al. [19] presented a literature review on EV battery recycling. 
The whole process from collecting batteries, over discharging, disassembly and material recovery was examined. The different material recovery techniques were also compared. Werner, Peuker and Mütze [20] described the complete recycling Process of EV batteries and compared the tree most common process flows. These include the routes with high, low or moderate temperatures. Diekmann et al. [5] described the LithoRec Process for EV battery recycling. The achieved material recycling rate was in the range of $75-80 \%$. The process was described as follows:

- Discharging and short circuiting for lowering the electrical hazard and recover electrical energy

- Disassembly of battery system and feeding peripheries (e.g. cables) to existing recycling processes

- Crushing of the battery cells or modules under an inert atmosphere

- Drying

- Separating

- Sieving

\subsubsection{Example of an EV Battery}

To give further illustration of the design of an EV battery, we took the 2017 Chevrolet Bolt battery as an example. The observations on the battery have been taken from the Videos of Kelly [21], [22] who performed a disassembly and re-assembly of that EV battery. Figure 3 shows the battery with the cover removed. The battery system is made up of 5 Battery Sections, with each Battery Section made up of two battery modules. The two modules in the front are referred to as Battery Section 1, the next two 
modules are Battery Section 2 and the next two are Battery Section 3. The lower two modules in the back are Battery Section 4, while Battery Section 5 is located above Battery Section 4. That two-level structure in the 2017 Chevrolet Bolt battery creates difficulties and requires extra efforts for disassembly. But the design with two levels helps to use the vehicles space more efficiently for storing electric energy and helps for providing a higher driving range [21].

The 2017 Chevrolet Bolt battery costs about $\$ 15,000$ and weighs approximately $435 \mathrm{~kg}$. The battery dimensions are approximately 1.6 meters long by 1 meter wide, while first three Battery Sections are approximately 15 centimeters high and the Battery Sections 4 and 5 are combined about 30 centimeters high. The Battery has total of 288 cells with Battery Sections 1-3 having an equal number of cells while Battery Sections 4 and 5 contain a little bit less Battery Cells [21].

In the front there is the orange cover for the relay assembly which covers the main electrical components. The long orange parts are the busbars that connect the battery modules. Around the battery modules just inside the tray are orange and black cables. The black cable goes all around the battery tray as a low voltage harness. The orange cables are the high voltage sense lines on each side of the battery. In the front of the EV battery there is an orange electrical connector. On the top of battery section 5 the orange high voltage disconnect can be seen next to the black battery energy control module (BECM). Between the modules and in front of the Battery Section 1 metal brackets are visible. Those fix the Battery Sections or modules [21]. 


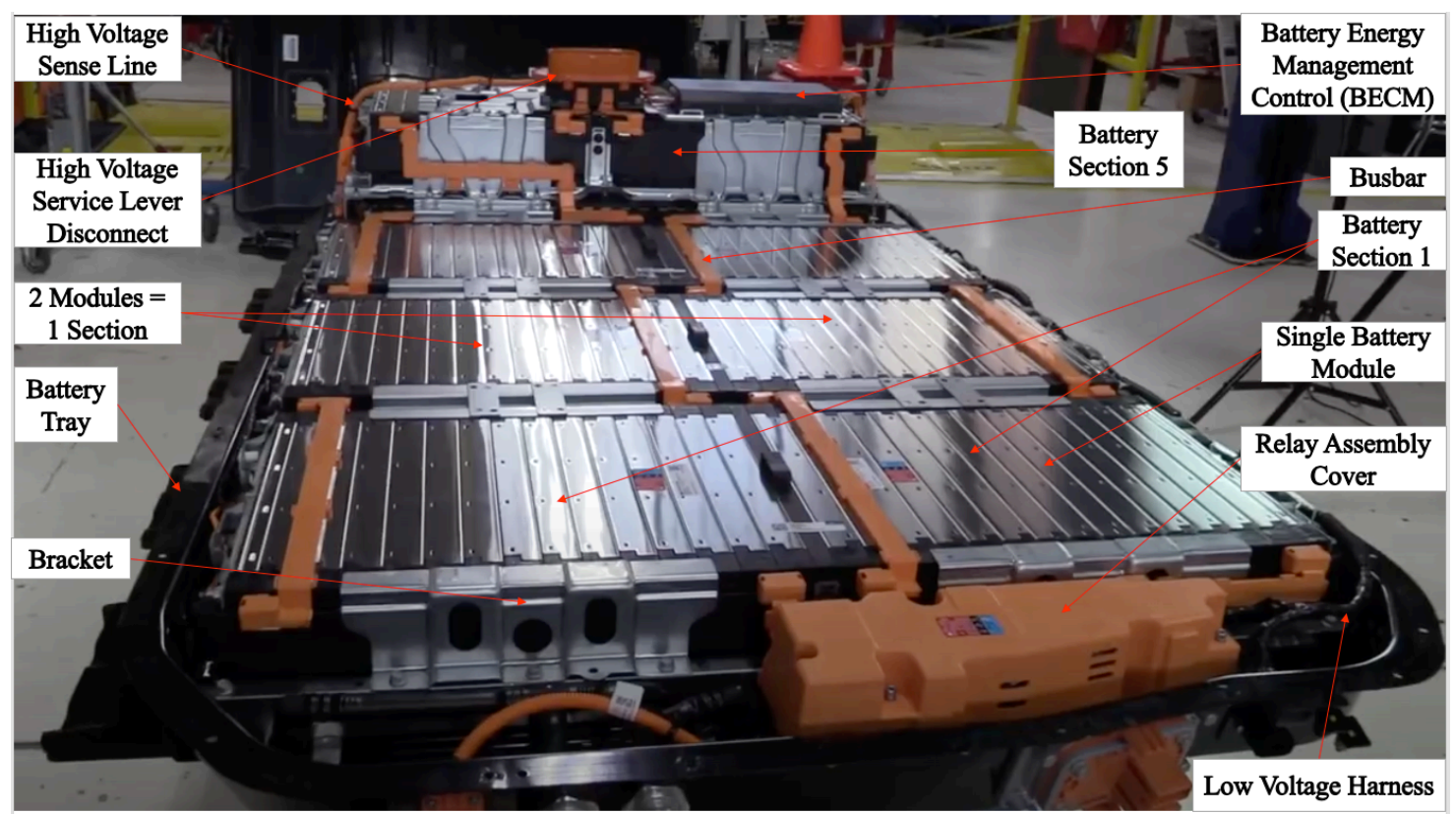

Figure 3: 2017 Chevrolet Bolt battery with removed cover, adapted from [21]

\subsection{Disassembly Strategies on E-waste and EV Batteries}

This section will review several studies on the disassembly of electronic waste in general. Following that, studies on structure of the battery and the assessment of single disassembly steps for EV batteries are presented. After that studies on the disassembly of EV batteries are summarized and the applied concept of human-robot collaboration will be presented.

\subsubsection{Investigations on the Disassembly of E-waste}

Since there are not many studies on the disassembly of EV batteries, studies on other recyclable objects were considered. Those studies [23]-[54] include disassembly planning, the optimization of disassembly sequences and the investigation of disassembly techniques. A detailed quantitative literature analysis was presented in [55]. Below is a 
review of few of these studies and several more are discussed later for assessing single disassembly techniques.

Li et al. [23] discussed selective disassembly for electronic equipment. The selection was based on economics and legislation or other stakeholder demands. Therefore, adaptive decision-making models with a multi-criteria basis have been developed. It was stated, that effective disassembly planning is necessary in order to raise the recycling rates of disposed electronic products. A case study on liquid crystal displays was performed. Twenty disassembly operations have been identified and a disassembly constraint graph (see Figure 4) was drawn from those. The disassembly operations are represented by the nodes, while the arcs illustrate the constraints. Using this, graph, different disassembly plans can be developed and compared. The disassembly graph for the 2017 Chevrolet Bolt (to be presented in the next chapter) was developed using a similar approach.

Starting with an initial plan, an algorithm optimized the disassembly sequence and depth of disassembly concerning different goals. Such disassembly goals include a fast removal of total weight of parts, hazardous material, value for a given time interval, total value, or total time. Those optimizations helped in order to fulfill the requirements of different stakeholders such as the most cost-efficient plan, the most environmentally friendly plan or the best plan for fulfilling legislative restriction. New restrictions, or changes in market prizes of the materials could also be adjusted. 


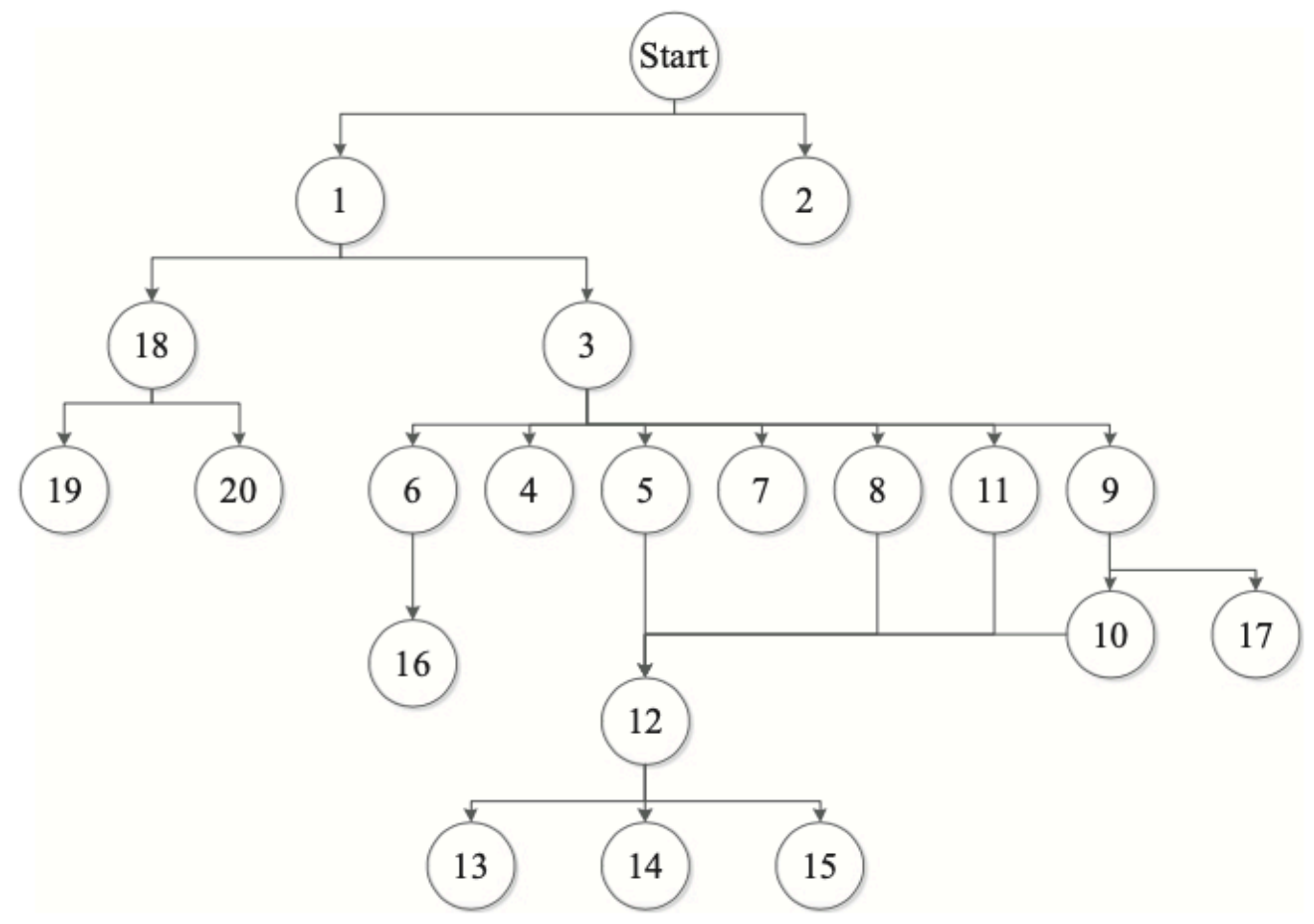

Figure 4: Example of a disassembly constraint graph for crystal liquid displays [23]

Wang et al. [24] also described an approach for selective disassembly planning and proposed using destructive disassembly for saving disassembly time. Kerin and Pham [35] described the impact of industry 4.0 on remanufacturing. A review on current research on the application of Virtual Reality, the Internet of Things or Augmented Reality on disassembly or remanufacturing was presented.

Hohm, Mueller-Hofstede and Tolle [46] investigated the disassembly of electronic devices. They introduced a "Model of the environment" [46] (see Figure 5) that relates all the parts in the device. The parts were grouped into active and passive parts. Active parts are referred to as parts with a connecting character. Those could be screws, or other fasteners, while passive parts are the connected parts. So, in between two passive parts, there has always to be an active part [46]. 


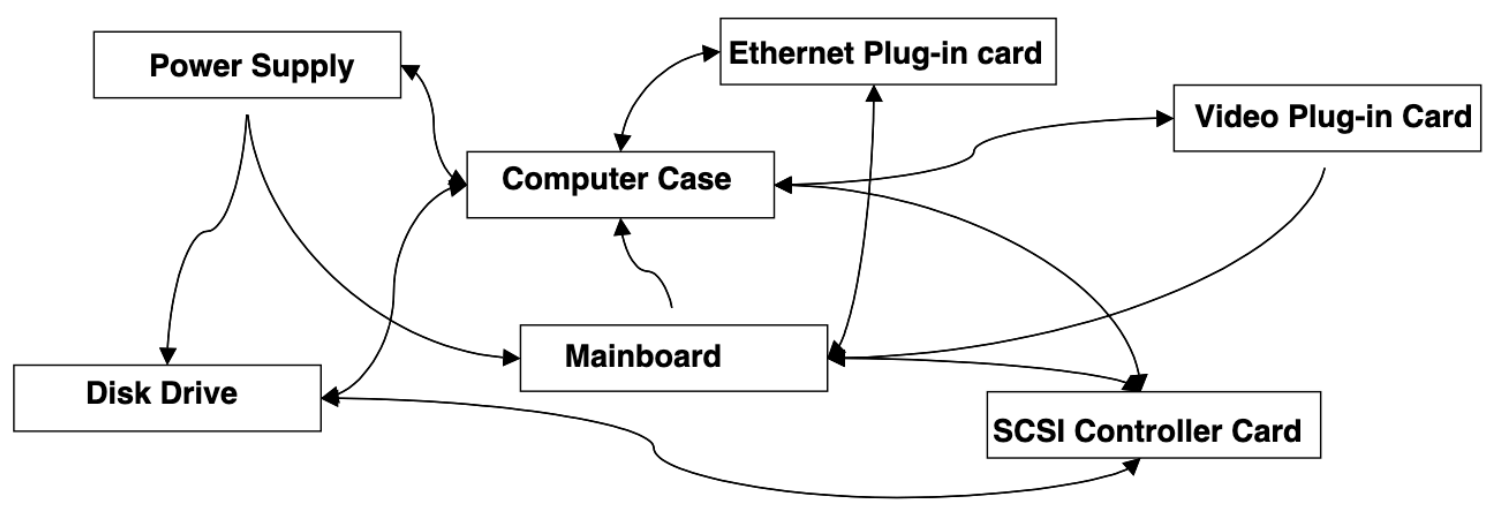

Figure 5: Graph model of the environment [46]

Lee and Bailey-Van Kuren [49] presented a model for automated disassembly with an included sensor-based supervisory control algorithm. Case studies on a single-use camera and a PC have been performed. Time and number of components for reaching the goal have been optimized. In order to minimize the time, tool changes are optimized. The algorithm was able to select the next component for disassembly from the knowledge on product design and the current component. An error recovery routine was also tested.

Tang et al. [50] described the modeling of disassembly processes. They introduced and compared different techniques for optimizing disassembly sequences. They used the example of a hand light device and drew a connection graph in order to determine possible orders of disassembly steps. From that, different graphs have been used for illustrating possible disassembly sequences: Direct graph [56], AND/OR graphs [56] and two types of disassembly petri nets [57]. Those graphs have been used to indicate possibilities for finding and optimizing disassembly sequences. Such techniques could be included into the planning algorithms of intelligent robotic disassembly work-cells. 
Vongbunyong, Kara and Pagnucco [38] described a system for automated disassembly dealing with uncertainties. Therefore, advanced behavior control with the cognitive abilities learning and revision was developed. A knowledge base was implemented for storing information that the cognitive robotic agent learned. The sensorics of the robotic system were connected to the disassembly planner. The collected information included the geometry, product structure the components and their quantity. The problem of non-detectable parts and fasteners was discussed. The cognitive robot with the knowledge base interfered with the human assistance and the physical world with the vision system and the robotic end-effector. For every recyclable product it was first determined if it is known or unknown. For known products the information from the knowledge base were used and revision took place for modifying the knowledge base. For unknown products the system devolved a disassembly plan by learning. That learning could be by demonstration of the human worker or by reasoning while executing operation plans. Experiments on LCD screens were taken to test the system. It was disassembled in a semi-destructive way with cutting operations. The recognition of parts was relatively accurate, but for fasteners, especially screws there were many false positives or false negatives. The learning allowed the system to work efficient and autonomous after some revisions.

Feldmann, Trautner and Meedt [41] summarized the German legislation on the management of waste and described concepts strategies for efficient disassembly. Furthermore, a concept for a gripper and a splitting tool was presented. 


\subsubsection{Structure Analysis of EV Batteries and step Assessment in Disassembly}

Wegener et al. [58] investigated the disassembly of EV batteries using as an example the Audi Q5 hybrid (see Figure 6). The relatively small HEV battery consists of

four Battery modules and weighs $35 \mathrm{~kg}$. They suggested to discharge the batteries first and then disassemble the batteries in order to sort the parts and materials. The most important parts are the battery cells. A shredding is suggested for the battery cells to regain the valuable materials such lithium and copper and to reuse disposed electrical parts. Fourteen main parts have been identified. A table was created where each part scored with the numbers of predecessors in disassembly. In that way an order for the disassembly was developed. Each step was described with the corresponding tool for manually disassembly. From that point, a disassembly priority graph was developed and succeeding steps with the same tool have been combined.

Furthermore, based on the analysis of the battery system parts more challenges have been mentioned. At first, there are different types of fasteners, so a time-consuming tool changing is necessary. Additionally, since the fasteners are accessible from different directions, changes in the directions of the robotic end-effector are also necessary. Due to the large variety in designs and the difficulty of some steps a human-robot collaboration was proposed where a Lightweight Robot (LWR) assists a human worker. For the relatively low complexity of the hybrid battery system with a low number of parts, it was suggested to finish the process on a single workstation. In the proposed system, the main task of the robot will be the identification and loosening of screws. Four categories of disassembled parts have been proposed: Battery modules, metals (with iron), electronics and residual materials. 


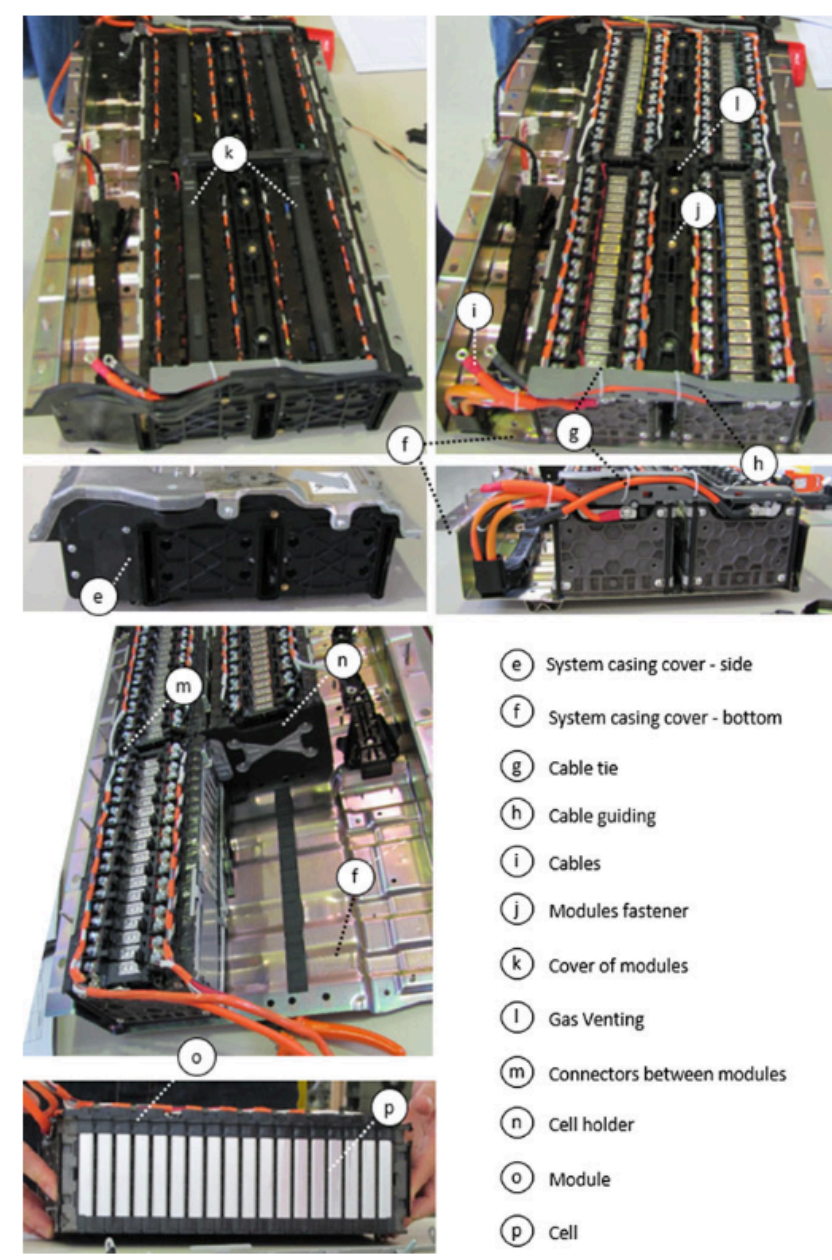

Figure 6: Design and parts of the Audi Q5 hybrid battery [59]

Herrman et al. [60] took a closer look on the automation potentials of single disassembly steps. For that purpose, a product analysis was done, and a criteria catalogue was developed. The product analysis was based on many different battery systems including BEVs, PHEVs and HEVs. A software tool has been used to collect the information about disassembly sequences, part costs and disassembly times. From that information the development of a disassembly graph took place. In the disassembly graph information such as the disassembly times for single steps are stored. Fifteen main disassembly steps for disassembly down to the level of battery cells have been identified. 
In order to assess the automation potentials for the disassembly steps, two indicators have been developed for counting scorings. The first is the "technical ability of a disassembly process to be automated" (TAA) [60] which is used to access the possibility to automate a step from a technical point of view. The second is the "necessity to automate the corresponding disassembly operation" (NA) [60] which describes the economic feasibility to automate a single disassembly step. Twelve criteria for NA and eleven criteria for TAA were created for the scoring model and weighted differently based on the importance of each criterion. With that scoring model each disassembly step could score from -100 to 100 points in total in each category. Therefore, on each criterion a scoring from -1 to 1 was possible and the different weight factors made it possible to sum up to a maximum of 100 . For example, regarding the TAA, a value of 1 would mean an easy realizable automation, 0 would mean indifference and -1 would mean that automation realization would be difficult. For joining techniques, a scoring of 1 included crammed connections, a scoring of 0 included screws and -1 glued joining techniques. The economically driven NA included categories such as weight, or the number of disassembly motions, and also cost related and safety criteria. As an example, there is a higher necessity to automate a step, if heavy weights have to get carried by human workers, or they have to protect themselves against dangers (e.g. high voltage) or a step, that includes many motions and is time consuming and costly if it is done manually.

Based on that portfolio analysis a scatter diagram was developed. Figure 7 shows that scatter plot. It can roughly be separated into four categories (or quadrants) of disassembly operations. The first quadrant includes steps that are automatable and need to be automated with positive values for NA and TAA. The second quadrant includes steps 
that need to get automated but are not easily automated. Such steps have a positive NA, but negative TAA. Steps with a negative TAA and NA (third quadrant) should be done manually and steps with a negative NA but positive TAA (fourth quadrant) do not have to be automated, but it is relatively easy (TAA) to automate those.

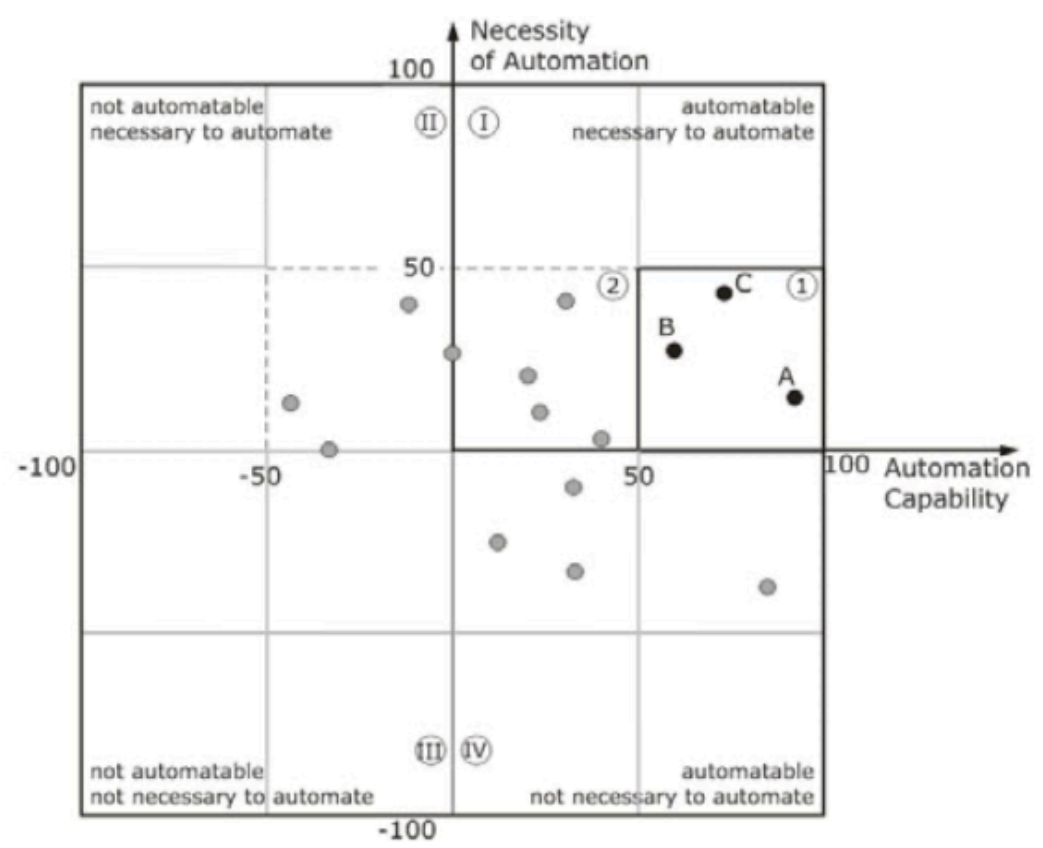

Figure 7: Results of the portfolio analysis plotted in a scatter diagram [60]

From this analysis it was suggested to definitely automate the three steps (A, B, C). Those are the handling of the battery system to the disassembly area, the extraction or lifting out of single battery modules and the extraction of single lithium cells. It was proposed to do all other steps manually [60].

Comparing the results from Hermann et al. [60] and Wegener et al. [58] there is a difference in the assessment of some operations and steps. Wegener et al. [58] proposed the loosening of fasteners as the main task for the robot because of the high repetition in that task. On the other hand, Herrmann et al. [60] counted screws only with an TAA scoring of zero. So, they proposed to only automate handling and repetitive grabbing 
operations. Part of this difference could be a general larger focus on unscrewing operations by Wegener et al. [58].

Li, Barwood and Rahimifard [61] presented an assessment for robotic disassembly using multiple criteria. Environmental, technological and economic criteria were taken. Formulas were developed for accessing the three categories. The validity of the assessment was tested with a case study where three different electronic components from automotive were assessed and disassembled. Each disassembly step was listed with the co-responding time and tool and the decision whether it was done automatically or manual.

Schwarz et al. [62] described an approach for collecting data about disassembly of EV batteries for optimizing it. A virtual disassembly tool was developed that helped to predict the disassembly time. The system gained information about material composition and disassembly time that can be used for research on EV battery disassembly.

\subsubsection{Disassembly Concepts for Electric Vehicle Batteries}

Wegener et al. [6] suggested a human-robot workstation where the robot and human share the same workspace for reduction of transport time. Each, human and robot have access to their own disassembly tools. As an example, the robot is proposed to do the relatively easy unscrewing task while the human worker performs the more complex prying tasks. The LWR is used to work together with a human. The robotic end-effector could be positioned manually by the human worker or with the help of a vision system. A Camera-based detection of screws was used. It was reported, that the detection of larger screws was more accurate than those of smaller screws. Fastener positions could also be 
demonstrated by the human worker. It was also mentioned that this manual demonstration of locations was time consuming and that accurate automatic location of fastener positions with a vision system would be much faster. A bit changing mechanism was proposed that allows it to unfasten different sizes and types (screws, nuts, bolts) of fasteners with the same robotic end-effector.

Schmitt et al. [63] stated that the automation of EV disassembly needs a high flexibility. Additionally, it was mentioned, that a fully automated disassembly is unrealistic and not efficient because there are many steps that are too challenging for automation. The barriers for automated disassembly are also summarized. Those are structured as product, process, environment, and logistic related. Examples of product related barriers are fasteners or a design that is not disassembly friendly (see definitions on design for assembly and disassembly (DfAD) by Boothroyd and Alting [64] and an evaluation of design for disassembly by Campbell and Hasan [65]). Process related barriers could originate from parts with an unstable form and location (e.g. cables that should be cut). The environment related barriers refer to usage and aging variance in the product or a non-optimal recycling area. Logistic barriers could be a missing labeling and in general the high number of EV battery variants. A high automation potential was seen for the extraction of the single disconnected battery cells out of the opened modules. Therefore, a flexible gripper was developed, that was also able to measure the state of charge of the cells in order to avoid high voltage (HV) dangers. That gripper is further described later.

Harper et al. [11] described challenges in EV battery disassembly and how automation could be performed. Some of these challenges include component sizes that 
are different in different battery designs and that there is a need for qualified employees because of the high weights and HV dangers. A main threat for automation is uncertainty. As a potentially useful algorithm for pre-sorting of batteries, the Optisort system (proposed by Chen and Shen [66]) was described by Harper et al. [11]. It uses computer vision algorithm for reading the labels and sorting batteries (currently only small consumer device batteries). Current algorithms can identify objects based on shape, size, texture and color. A further suggestion would be the labeling of the main components with QR-codes or RFID tags. It is stated, that there is a need for intelligent behaving robots and that therefore sensors are most important. Tactility and force-sensing are other major requirements. It was concluded, that re-use ("second life") is economically more feasible than direct disassembly, but disassembly following the re-use should be automated as far as possible in order to reduce risks to human workers. Therefore, the design must be adjusted to be more disassembly friendly.

Maharshi and Janardhan [67] described the prospect of using cloud computing in the disassembly of EV batteries. Sensors help to assess disassembly parameters such as size, weight and materials. All collected data will be exchanged by cloud computing. A suitable disassembly program for a single battery type will be chosen automatically from a library. Kampker et al. [68] compared different layouts for a disassembly plant of remanufacturing EV batteries. The layouts included linear U-shape, S-shape and L-shape factory layout for the different disassembly stations and performance parameters such as disassembly cycle times or the needed space were compared. Kay et al. [69] investigated the automated disassembly of EV batteries. Technicians were observed on their manual 
performance of disassembly. Experiments on gripping and cutting operations were performed. Simulation of path planning was used for programming an experimental robot.

\subsubsection{Human-Robot-Collaboration}

As discussed before, human-robot collaboration is a promising concept for disassembly and especially for the complex and unpredictable disassembly of EV batteries. In this section, human-robot collaboration will be further defined, and different aspects of human-robot collaboration and the usage in EV battery disassembly will be discussed.

Goodrich and Schultz [70] described human-robot interaction (HRI) in general as robotic systems that are used by a human or where a human and robot work together. The biggest distinguishing factor for HRI is how the robot and the human communicate and if there is close proximity between them. Remote interaction means that there is a spatial or temporal separation. Proximate interaction means that the robot and human share a location. Examples could be a service robot working in a hotel or industrial robots assisting humans.

Murata [71] described human-robot-collaboration as the opportunity to combine the advantages of humans and robots for accomplishing different task and compensating each other's weaknesses. The advantages and disadvantages of humans and robots are explained. Robots are good in fast, accurate and repetitive tasks and can operate in hazardous environments, while they lack flexibility, communication skills and openminded thinking suited for creative problem solving. On the other hand, humans are not that reliable in performing repetitive tasks with a time-constant accuracy and are not 
suited for monotonous work due to ergonomics aspects. Other problems are an imprecise memory and a lack of persistence of human workers.

Lotter [72] described hybrid assembly systems. Those systems can be seen as a stage in between manual and fully automated assembly. Figure 8 compares hybrid assembly to automated and manual assembly. Automated assembly is good for high quality and high productivity but lacks flexibility and can only handle a low number of variants. Manual assembly has a low quality and productivity but is more flexible and can handle a large diversity of variants. Hybrid assembly is a compromise of the advantages and disadvantages of both and suitable for cases where manual and automated assembly are not suitable.

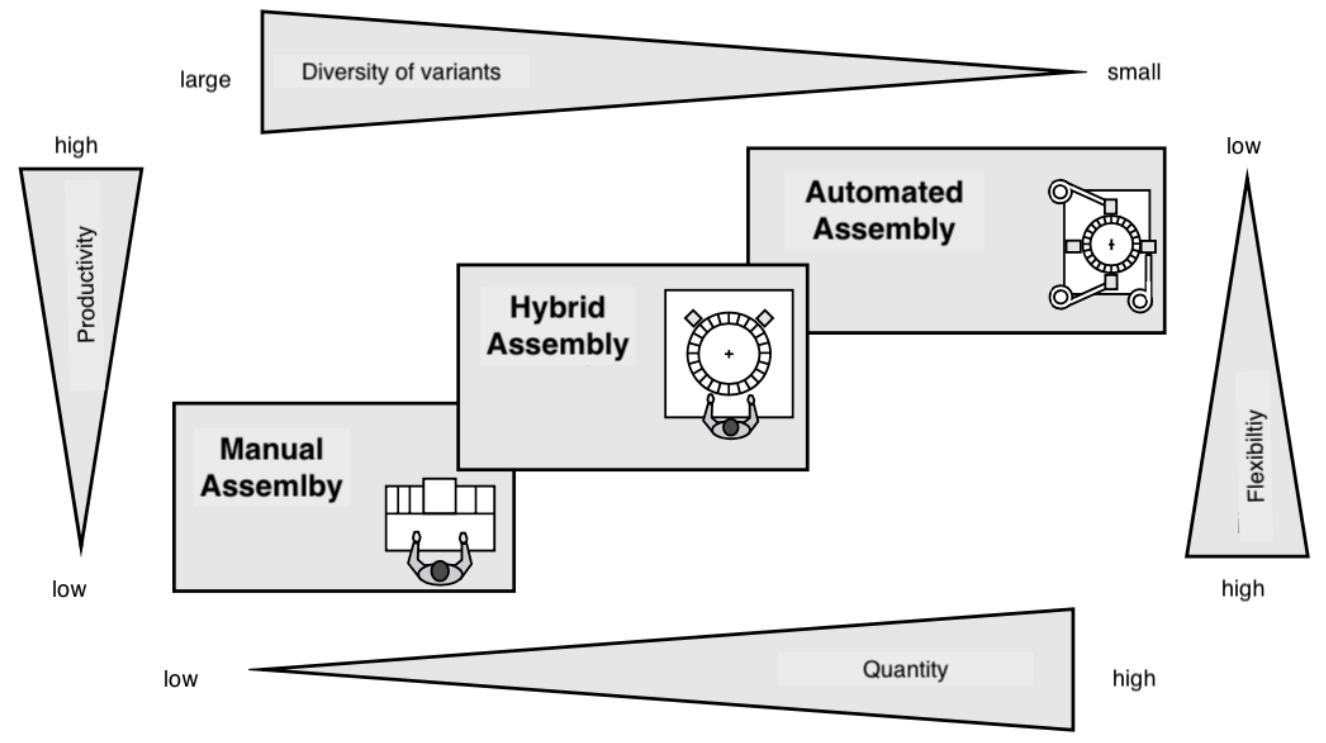

Figure 8: Classification of hybrid assembly, adapted from [72]

Wegener [10] concludes, that the disassembly of EV batteries is such a case where hybrid assembly or in that case hybrid disassembly is suitable. It was assumed that there will be a high number of EV batteries requiring disassembly. That indicator would argue 
for automated assembly, but the large diversity of variants and the need for high flexibility suggest a manual assembly. After combining those two aspects, the hybrid assembly that includes human-robot collaboration with a high proximity seems appropriate [10].

Another aspect in human-robot collaboration is robotic learning. Algorithms are implemented that help the robots learning and improve skills from the processed data. Furthermore, there is the aim for accomplishing direct teaching by humans [70]. Argall et al. [73] discussed learning by demonstration where the human shows the robot how to accomplish a task. The goal is that the robot interprets the human movements and develops own actions for doing the task, but not exactly imitates the human motions.

Collision preventing is also an important aspect in human-robot collaboration. Gecks [74] describes a system of cameras and online path planning in order to avoid collisions. Cameras are mounted above the workspace. The distance between human and robot was used to adjust the speed of the robot. That helped to achieve fast robotic working if the distance is safe enough and the robot moves with safe and slow motions for closer distances to the human worker [74]. Another collision detection method without external sensors was presented by De Luca et al. [75]. Proprioceptive sensors of the robot were used for collision detection and different reaction strategies were discussed. Vongbunyong, Vongseela and Sreerattana-aporn [76] described how the expert knowledge of human workers can be transferred to the robot. A case study on LCD screens was performed. Zhang et al. [77] described, how neural networks can be used in order to predict the human motions in order to achieve human-robot collaboration. A case study with the assembly of an engine was performed. 
Bdiwi et al. [27] used a robotic work cell with HRI for the disassembly of electric vehicle motors. An active cooperation with a teaching and a cognition phase was developed. A vision- and force-sensor concept was implemented for human safety. In the teaching phase the robot tracks the human hand with the help of the vision system. When the robot reaches a defined force control zone, the human informs the robot by waving that a phase of physical interaction starts. The human teaches the fastener positions manually and the robot builds a base of knowledge from that. After that in the cognition phase the robot uses the developed knowledge base on different motor models to remember fastener positions or locating those with a vision system. It was concluded that in the proposed system human and robot interact safely and the robot can respond to human actions.

\subsection{Proposed Concepts for different Disassembly steps}

This section summarizes approaches on robotic end-effectors and automation of single techniques that are necessary for EV battery disassembly. Such tasks include fastener and part detection with vision systems (2.3.1), unscrewing operations (2.3.2) and the necessary bit- or tool changing (2.3.3). Furthermore, grabbing (2.3.4), or prying and cover opening (2.3.5) and cutting operations (2.3.6) are discussed. Finally, studies on the layout of disassembly layout (2.3.7) are assessed.

\subsubsection{Detection of Fasteners and Parts by Vision Systems}

As discussed by [6] and [78] (see sections 2.2.4, 2.2.3 and 2.3.3) the locations of fasteners could be taught manually using the concept of a human-robot-collaboration. With that approach a human worker teaches the positions of all parts and fasteners by 
moving the robotic end-effector to their coordinates. The robotic system stores the position data and can reuse them if the same model will be disassembled a second time. This technique requires a high precision in positioning the disassembled object and there is a low tolerance regarding damages and variations.

In order to achieve a higher degree of automation it seems necessary to implement an automated tool for recognizing and locating parts and fasteners. Such a tool could be a vision system with one or more cameras and a data storage for identifying different types of fasteners and parts.

DiFilippo and Jouaneh [52] proposed and tested a concept for the automated removal of screws from the backside of laptops using a camera system. Two Microsoft Lifecam 3000-HD are used, with one camera placed above the whole system for locating circles that could be screw positions. Another camera was placed on the robot that helped for finding the screw holes and centering them. A system for calibrating the camera using a checkered square was also described. Because the resolution of the top camera is not good enough, a second camera on the end-effector was used for locating the screw holes accurately. Figure 9 shows the proposed system from the view of the top camera and shows the coordinate transformation.

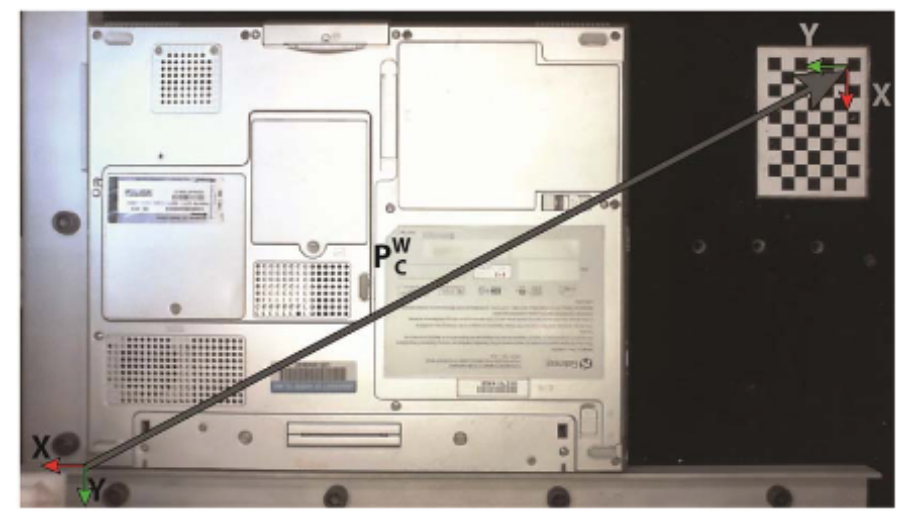

Figure 9: View of the Vision System for laptop recycling [52] 
A computer vision algorithm was used. It applied Gaussian blur and Prewitt edge detection on the taken images in order to find the screw holes. It searches for circles that could indicate screw holes. In the case that such a circle was found, the second camera was used for centering it. Then, an automated screwdriver was applied for unscrewing. Several trials with different laptop colors and brightness levels have been performed. In half of the detected holes, there was a screw present. The presence was proven by an accelerometer or current monitoring.

Gil et al. [53] used visual detection for a flexible multi-sensorial system that was used for automatic disassembly of electronic devices such as PCs. It was stated that there are two options for visual detection. One is to use grey values and known patterns. The other is to use contour characteristics for determination of bi-dimensional geometry models. The position of each component was computed, and the types of components were known. The robot knew how to approach each part and prepared for the current disassembly task with the right tool for each operation. It is stated that changes in the lightening of the area or views from different points can change the characteristics or objects can be hidden. A Gaussian mask was applied on the images and the contours are detected. After that, the Douglas-Peucker's algorithm created a polygonal fitting, and the edge points are determined by the progressive probabilistic Hough transform. With the help of those techniques the regions of screws were detected.

The vision system used by Gil et al. [53] was based on the approaches by Torres et al. [54]. That system aims for the recognition of parts as well as their location. Two cameras are placed on a y-z Cartesian robot. They overlooked the worktable form a top view and were movable due to the placement on a robot. It was possible to take images 
from more different positions and there were marks on the worktable for calculating fastener and part positions. A data base was used for determining recyclable parts based on geometrics and the relationships of the components. The system worked semiautomatic and some degree of human-robot interaction was necessary.

Yildiz and Wörgötter [79] presented an advanced approach using two neural networks for screw detection on computer hard drives. Many different screws with variable shapes and sizes were detected. Zazar Gandler et al. [80] presented an approach for estimating the object shape with the help of a visual and tactile data. Different types of objects could have been distinguished.

Hohm, Mueller Hofstede and Tolle [46] used a vision system in order to create a model of environment of electronic devices. A camera was positioned on one of two robots. Furthermore, a laser range finder was used for determining distances. All recognized parts were implemented into the structure of the environment. That structuring is further discussed in section 3.1.2.

\subsubsection{Unscrewing operations}

Wegner et al. [6] used robotic unscrewing for the disassembly of EV batteries. Additionally, the concept of human-robot-collaboration was applied (see section 2.2.4). It was described that one challenge is the variety of different fasteners in EV batteries. Therefore, a bit changing tool was developed (see section 2.3.3). The procedure of automated unscrewing can be described with four steps:

- Preparing the tool with the fitting bit

- Approaching the correct fastener position with the robotic end-effector 
- Engaging of bit and fastener with the help of searching motions

- Rotating the fastener until separation from battery is reached

The searching motions were described by Nave [25], which involve a slow speed rotation of the screwdriver until bit and tool engage. Wegener et al. [6] mentioned, that it is not realistic to use exact information about fastener location (e.g. CAD models) because those are normally unavailable. Therefore, the techniques of user demonstration and detection by a vision system were applied. Figure 10 shows the automated screwdriver.

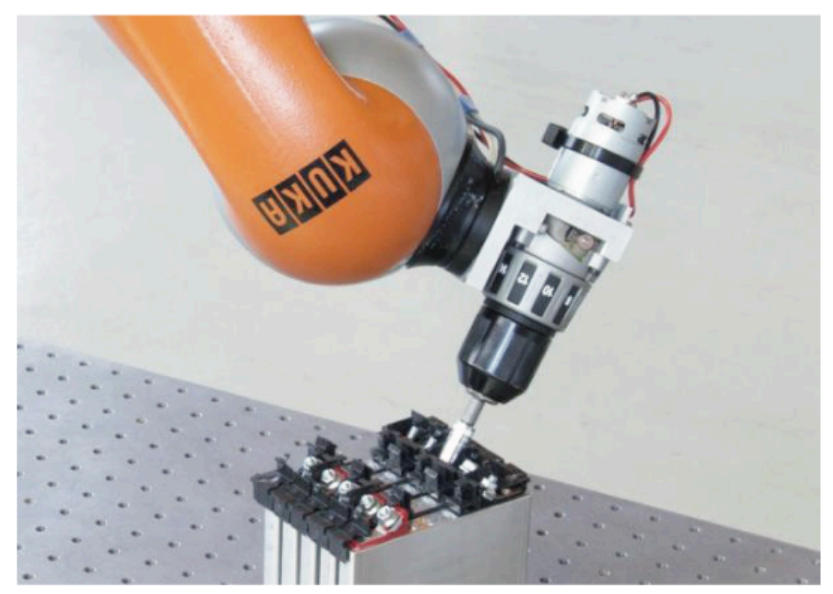

Figure 10: Robotic end-effector with automated screwdriver [6]

DiFilippo and Jouaneh [52] proposed a sensor-equipped screwdriver that was used for automated screw removal from the back of laptops. The screwdriver was combined with a vision system where the vision system provided locations of holes and the screwdriver tested if there was a screw and removed the screws. A sketch and prototype of the screwdriver can be seen in Figure 11. A low friction slide connects the inner and outer shell of the screwdriver, allowing relative motion between the tip of the screwdriver and a force sensing resistor (FSR) located at the top of the inner shell. The screwdriver moves to the possible screw positions detected by the vision system and approaches the 
screw by lowering the end-effector. After touching the screw, the FSR is triggered and the screwdriver checks for a possible screw.

Furthermore, an accelerometer was used to signal if unscrewing was completed successfully. That means, if the screw has no more connection to the screw hole. The screwdriver was driven by a DC motor. An electromagnet connected to the screwdriver was used for picking up/releasing the screws. The results showed, that the system was able to detect more than $90 \%$ of the screws.

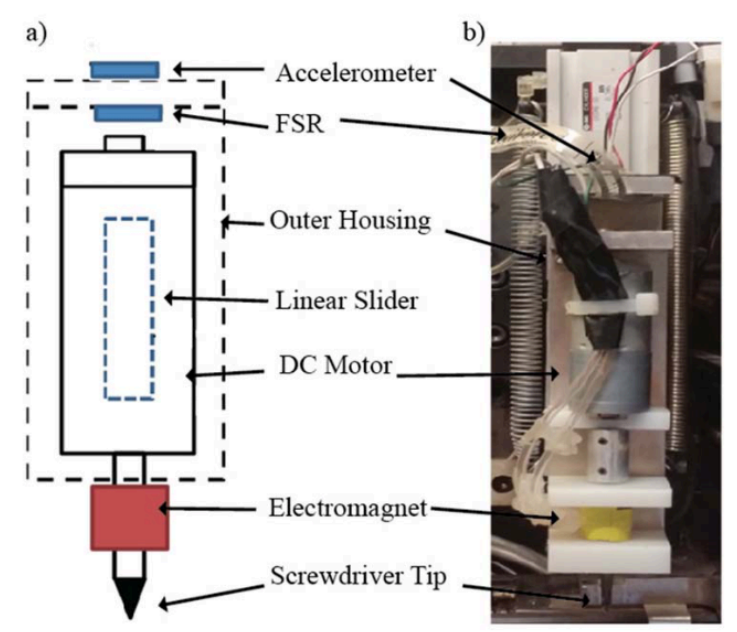

Figure 11: Sketch (a) and prototype (b) of an automated screwdriver [52]

Kristensen et al. [81] presented an approach for using reinforced learning (RL) for unscrewing in the disassembly of electronic waste. With the software Gazebo and middleware ROS a simulation was created where a UR5 robot with an automated screwdriver disassembles screws. The RL algorithm used the state on a force-torque sensor, the screw bit joint value and the position of the end-effector or the UR5 robot as inputs. By different signals the end-effector could move in positive or negative $\mathrm{x}, \mathrm{y}$ and $\mathrm{z}$ direction or rotate the joint for unscrewing. Penalties or rewards are given to the RL agent of the RL system based on how good the end effector approaches the screw in a training 
session. Figure 12 shows the simulation with the robot in its final position above the screw. The study provides an approach, how reinforced learning can be used for screw detection and unscrewing in disassembly.

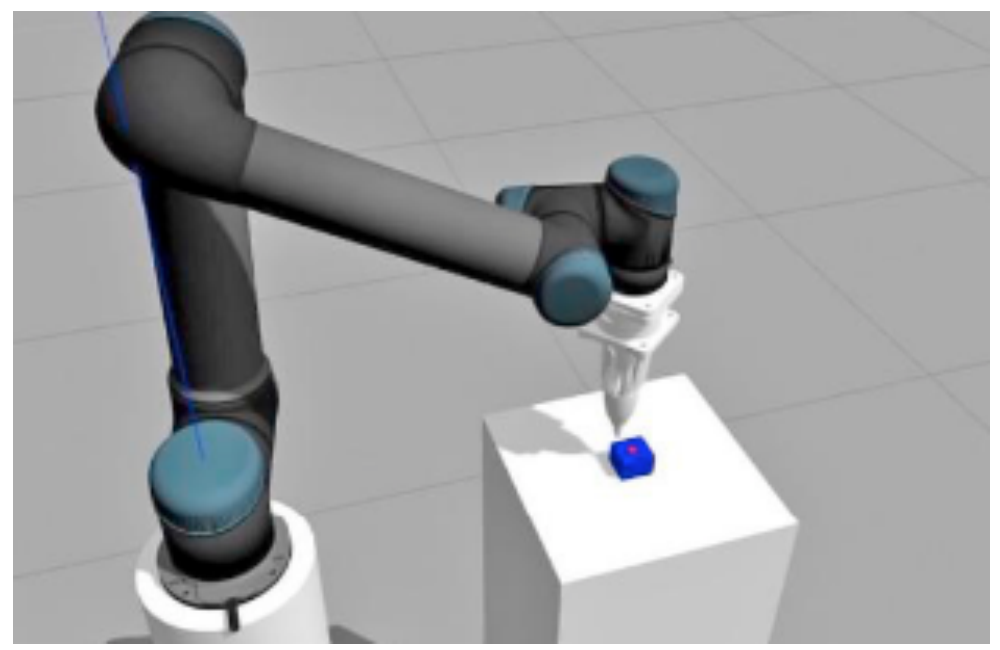

Figure 12: Desired final position of simulated robot for unscrewing with RL algorithm [81]

Li et al. [26] presented an automated nutrunner for hexagonal screws, where a collaborative robot was used for disassembling a turbocharger. The process of unscrewing was described. At first the nutrunner approaches the fastener position (from CAD models), then it uses a spiral search movement for finding the hexagonal screw head. After that a sensor is used to indicate if the screw head and nutrunner locked. In the moment of the locking there is a sharp increase of torque and the screwdriver changers direction of rotation for unscrewing. While unfastening it was observed that if oscillations take place, which would indicate, that the screw was unfastened successfully. Experiments indicated that there are shorter searching times for larger screws. Even with initial position errors, $98 \%$ of the tested screws were removed successfully.

Nave [25] investigated the automated separation of threaded connections with different techniques for disassembly. It was mentioned, that connections can also be 
destroyed, if unscrewing is not successful. That could occur, for example, if fasteners are corroded. A special unscrewing tool was developed that combined unscrewing and boring. Furthermore, due to the destructive disassembly tool changes could be avoided for saving time and investment costs. In general, it was described, that there are three ways of disassembling threaded connections:

- Non-destructive disassembly

- Partly destructive disassembly

- Destructive disassembly

Non-destructive disassembly is performed by unscrewing with a screwdriver. An example of partly destructive disassembly is milling of the head of the screw. Destructive disassembly is performed by milling of the complete screw connection or hollow-core drilling. Eleven indicators were classified that helped for determining the optimal approach for the separation of the threaded connection. Those include the access, the size of the tool or the materials of the parts. The unscrewing process was described with three phases: Connection to the screw; the unfastening; and the turning out of the screw. For finding a screw, a spiral search algorithm was used and different techniques for the endeffector have been compared.

\subsubsection{Tool changing and Bit changing}

The disassembly operations of EV batteries consisted of many different tasks such as unscrewing, cutting or grabbing operations. Therefore, different robotic end-effectors or different robots are necessary to accomplish these. As discussed in [6] unscrewing is the most common and the most important operation for automation. There are different 
types of fasteners (screws, bolts, nuts) and those fasteners appear in different sizes and types, so automatic bit changer is necessary to include in an automated system.

Chen, Wegener and Dietrich [78] reported on an automatic bit changer. As shown in Figure 13, a socket wrench inside the bit changer can rotate slightly. In order to align the tool with a bit, the tool will be inserted with some force (direction of tool) into the socket wrench. The force is increased for achieving a full engagement of the tool and bit. For loosening the bit, the tool moves inside the holder as far as possible upwards. Then a holder inside stops the bit from moving with the tool and it is loosened. The position of the bit changer is taught manually using human-robot-collaboration. After that, the robot tests the position with attempting to pick up a bit. The robot saves the learned positions to fulfill the bit changing operation automatically for the next operations.

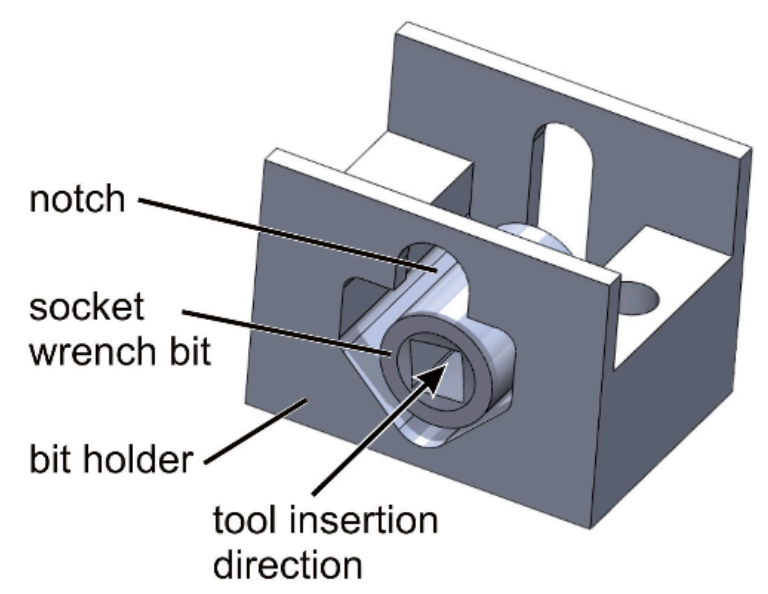

Figure 13: Automatic bit changer [78]

Gil et al. [53] also presented a tool-changer for robotic disassembly on different electronic devices that used human-robot collaboration. In their case, the tool-changer was able to change the tool, employed by the robotic end-effector for an application that involves circuit disassembly from a toy. After the detection of the circuits, the pliers cut 
the wires. Following that, the tool-changer exchanges the pliers with a screwdriver. In the end, the tool-changer chooses a tool for loosening the clamps to finish the disassembly process.

Nave [25] discusses the economic effects of tool-changes in the background of disassembly of electronic devices. In general, it was stated different kinds of fasteners lead to an increase of disassembly time and additional costs. It was pointed out, that destructive disassembly of screwed connections with the technique of counterboring the screws is faster automatically than manually. This technique should be used for screws with damaged heads. It was also mentioned that different screw sizes and automated toolchanging or bit-changing systems cause a higher system complexity and decrease profitability. By a product analysis in the field of electronical devises it was discovered that those devices often only use one or a few types and sizes of screws. Therefore, less or no tool-changes are necessary. Unfortunately, that is not true for EV batteries where many different kinds of fasteners appear.

Bdiwi et al. [27] proposed a tool-changing system that could change different types of nutrunners. It was used for the disassembly of EV motors with a cooperative robot (see section 2.2.4). The system included a store fixture, an universal tool and a switching disk.

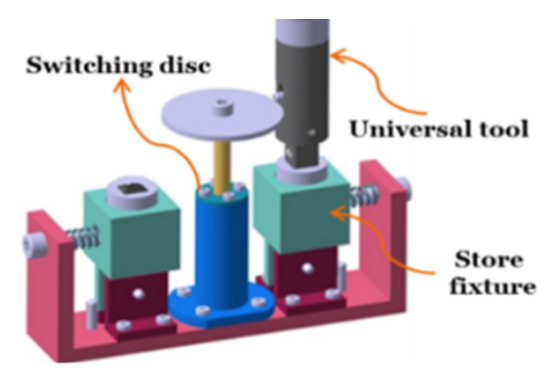

Figure 14: Switching fixture for tool change [27] 


\subsubsection{Grabbing operations}

Following the unscrewing of fasteners, in many cases a grabbing operation has to take place. EV batteries contain a large variety of different parts that have to be removed from the battery in order to reach the battery modules and battery cells. Those parts come in many different sizes, shapes and weights. They range from small electrical parts, long cables, medium size brackets to large and heavy parts such as large covers or cooling plates. In order to remove all these parts, the automated workstation needs strong, flexible and accurate grabbing tools to perform the combination of those requirements.

Weigel-Seitz et al. [28] investigated different grippers used in an automated workstation for the disassembly of electronic devices. A two-finger gripper and a threefinger gripper have been used. The three-finger gripper (see Figure 15) uses three rotational joints and has two levels of control structure. The lower level of the gripper's control structure used torque control, while the upper level used stiffness control for grip coordination.

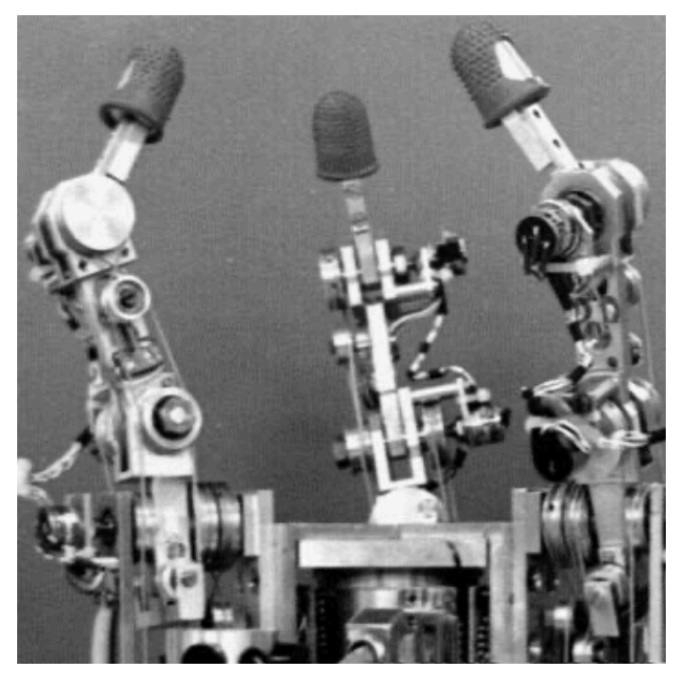

Figure 15: Three-finger gripper for disassembly [82] [28] 
The two-finger gripper (see Figure 16) was composed of two small fingers, so that it could reach into small gaps. Infrared sensors and a pressure plate are integrated in the fingers. Gripping force is measured with the help of the pressure plate and the infrared sensors are used to detect if there is an object in the middle of the two fingers. It was concluded that the three-finger gripper is not reliable enough and the two-finger gripper is not accurate enough for the usage in large scale automated disassembly and improvements in robustness are needed.

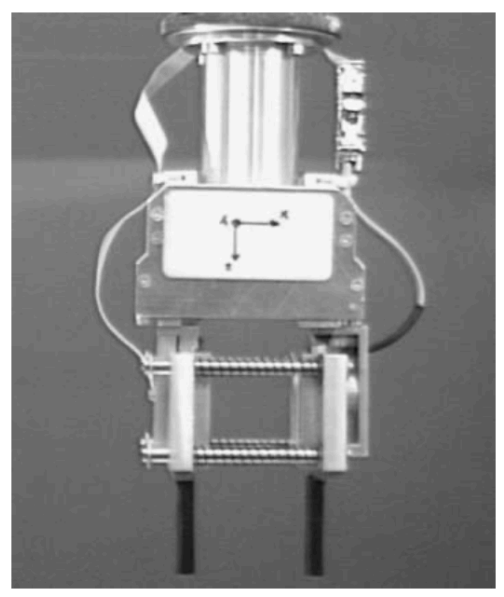

Figure 16: Two-finger gripper for disassembly [29] [28]

Borràs et al. [83] presented a single arm gripper that could be used in multifunctional ways. It was designed for the disassembly of electromechanical devices. Above the gripper there is an end-effector that could take different tools such as an unscrewing tool, pulling tool, scraping tool or leveraging tool. An automated tool-changer was provided. A prototype was designed, and its CAD model presented. The concept was tested by simulation.

Adjigble et al. [84] presented a grabbing tool for arbitrarily shaped objects. The system works without physical information or training for single objects. It finds a way to 
maximize the contact surface of the gripper and object. A 3D camera was used for estimating the object. Certain objects could have been classified by the algorithm. Marturi et al. [85] showed how grasping of moving objects could be realized. That case is important for HRI where objects are handed to the robot by a human worker.

Stenzel [30] investigated different types of grippers for usage in disassembly. He defined five different sub-systems in a gripper: Information (processing the data about the task and geometry), energy (pneumatic, electric or hydraulic actuator), kinematics (transmission and mechanical systems), force (aligning the gripper to the object) and carrier (integrating the gripper into the overall disassembly tool). Grippers have been invented that bore into the materials of the parts. One example is a gripper for plastic parts (see Figure 17) that uses headless screws that were bored into the parts. Once a stable connection was achieved, the robot lifted up with the disassembled part.

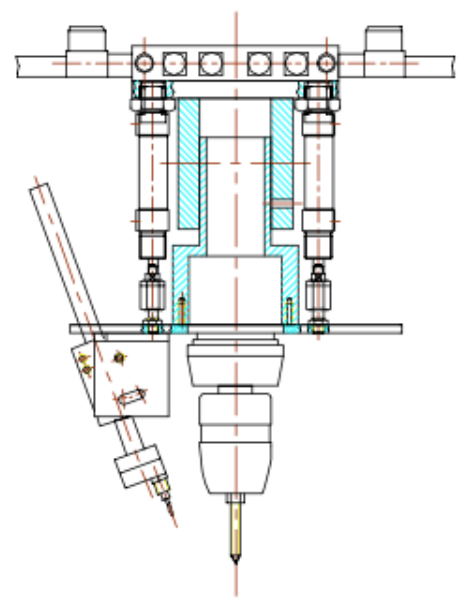

Figure 17: Sketch of a gripper boring into disassembly parts [30]

Schmitt et al. [63] introduced a flexible tool for extracting battery cells from EV batteries that consists of two parallel two-finger grippers (see Figure 18) that are placed on profile rail. One of the jaws is fixed while the other is movable for easy adjustment to 
different battery cell geometries. Additionally, one of the contact plates of the grippers is conductive and the other is not, so the voltage of the cells could be measured to report the status. The motion of the gripper on the rail is done through a DC-motor.

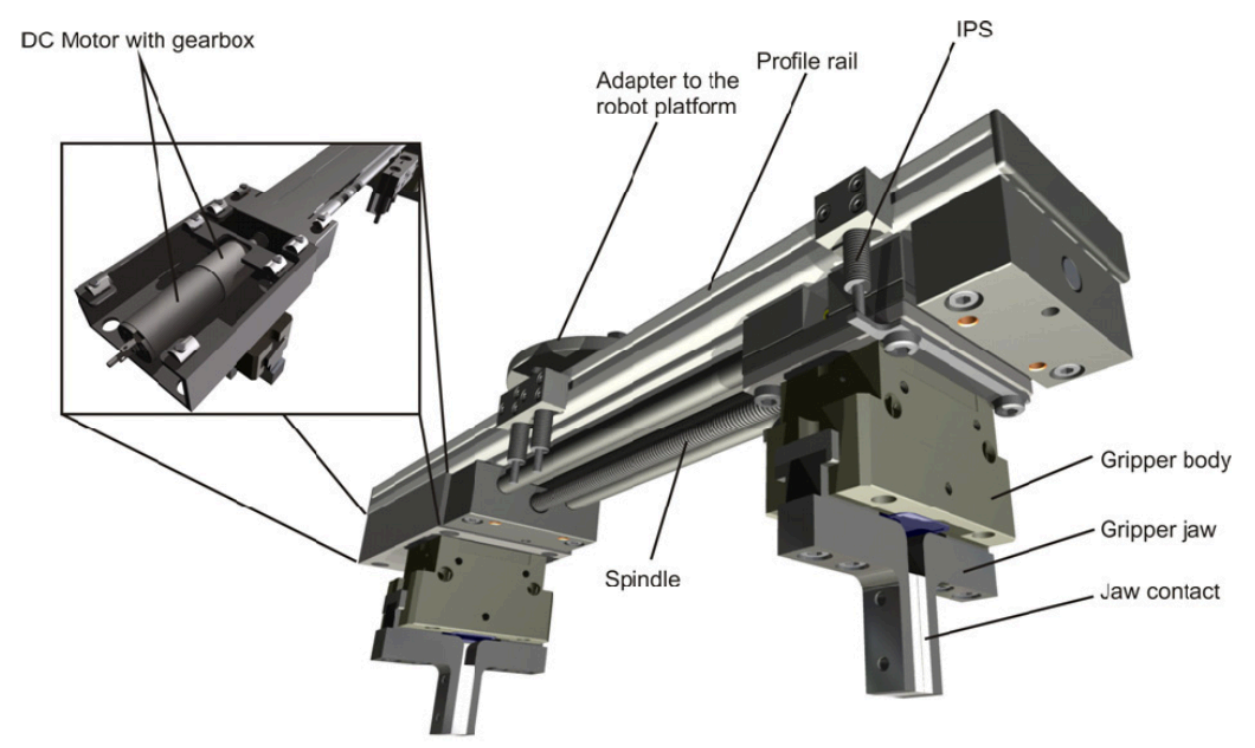

Figure 18: Gripper system for EV battery cell extraction [63]

\subsubsection{Prying operations and Cover opening}

The opening of covers can be an important task in the disassembly of some EV battery types. As discussed in (2.1.1), the design of the 2017 Chevrolet Bolt battery includes many covers such as those that cover the nuts that fix the busbars. Because there are many of those covers, the operation is relatively repetitive. Therefore, it seems useful to have a robotic end-effector tool that can perform this operation.

Schumacher and Jouaneh [31] worked on the design of a disassembly tool for opening the cantilever snap-fit covers of small electronic devices (e.g. TV remotes). They also included a mechanism to extract the batteries after opening the covers. Their tool makes use of inexpensive FSRs to provide force feedback information. In a FSR there are two membranes separated by a thin gap of air. One layer has a conductive material while 
the other layer partly consists of ink that is pressure sensitive. If a force is applied to the FSR, there is a decrease in resistance that can be measured through the use of a voltage dividing circuit. Those voltages are measured and converted into units of force through a calibration procedure. The generated information from the FSR have been used to control the motion of the tool tip.

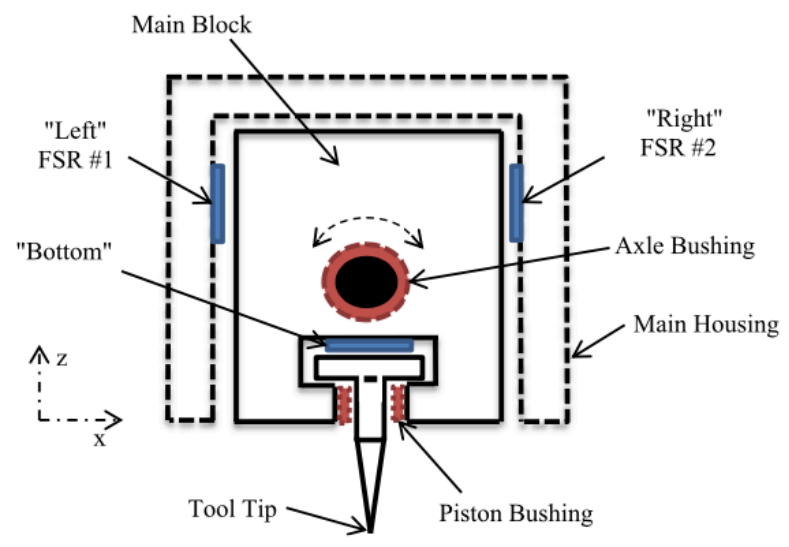

Figure 19: Prototype design of cover opening tool [31]

The discussed prototype is shown in Figure 19. It includes the force sensing tool tip that could move in different directions. The cone shape of the tool tip enables it to do both tasks, the opening of the snap-fit-covers and the extraction of the batteries. In addition, a vacuum gripper was used to grasp the snap-fit covers after the tool released those. The proposed system was able to fulfill the cover opening and battery extracting tasks under various testing configurations.

\subsubsection{Cutting operations}

Gil et al. [53] used a cutting tool for cutting wires and electronic circuits in the disassembly of electric devises such as toys and PCs. Bailey-Van Kuren [32] described a robotic workstation for disassembly with an included cutting tool. A case study on the 
disassembly of mobile phones was presented. Figure 20 shows the disassembly work-cell with the cutting-tool. Cutting operations are described as operations where connections have to be dismantled and the valuable component is left undamaged. For disassembling mobile phones, the first operation is the use of a high-speed rotary tool with a saw blade that cuts around the perimeter of the phone for dismantling the cover. The second cutting operation is point cutting around the screws.

In the concept of human-robot collaboration by Wegener et al. [6] the cutting of cables was classified as a difficult task for the robot and should be carried out by the human worker. The flexibility of the cable ties was mentioned as a reason that makes it very complicated for the robot to fix and cut them. Gerbers et al. [86] also stated that the robot for disassembly of EV batteries should perform unscrewing tasks, while the human can do difficult operations such cutting. Harper et al. [11] explained that it is necessary to perform interaction of different robots for cutting operations. For example, one robot could fix the cable, while the other cuts it. Ortenzi et al. [87] stated that there is a need for simultaneous control of force and motion for robots performing such a task.

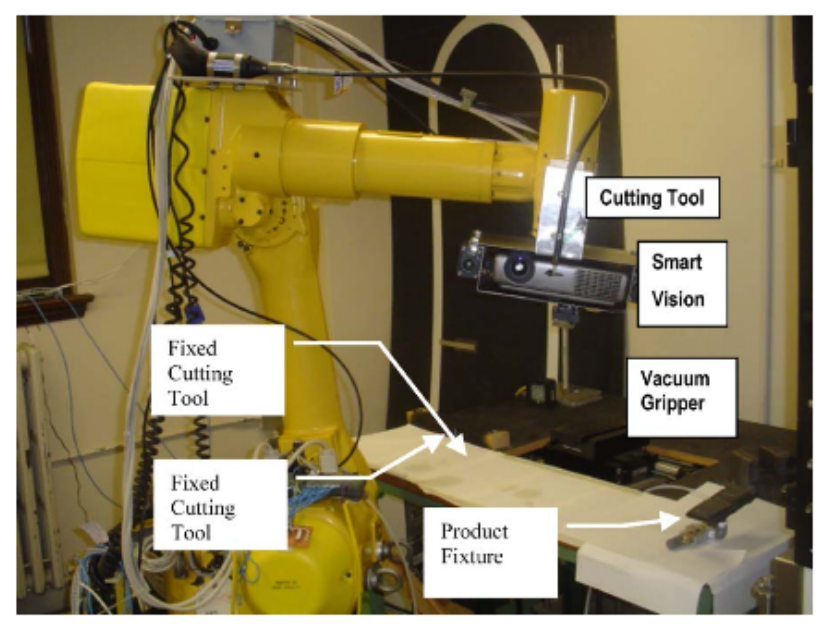

Figure 20: Robot for the disassembly of mobile phones with included cutting tool [32] 


\subsubsection{Disassembly Work-Cell layout}

Wegener's [10] workstation for the disassembly of EV batteries is can be seen in Figure 21. The workspace is shared by the human and the robot and is supervised by a sensor for the safety of the human worker. From a control point of view, the camera is the sensor for the disturbance variable and is used for collision avoidance. The sensor signals were processed for the disassembly control task. The disassembly control task processed the data of desired positions and actions to the engine control task that moved the robot, which in turn provided feedback to the disassembly operation. The used robot is a $L B R 4+$ designed by $K U K A$ that is suitable of cooperative disassembly. Another advantage of that robot is its low weight of only $16 \mathrm{~kg}$ and the compact design. That low weight combined with a mechanism that let the robot move back if it measures external forces helped to reduce the negative impacts of collisions with the human worker and ensured additional safety. The robot can handle a maximum payload of $7 \mathrm{~kg}$, and the universal flange can be equipped with different tools. The bit changing mechanism allows it to automatically switch bits for disassembly of different types of fasteners (see section 2.3.3). As a cooperative robot it is able to learn new workflows and positions by user demonstration (see further explanations in section 2.2.4) [10].

For supervision, the camera Xtion PRO LIVE by ASUS has been used. That camera implements an algorithm for recognizing humans which processes the outline of a human to a simplified skeleton. The recognition works for a distance in the range of $0.8 \mathrm{~m}-3.5 \mathrm{~m}$ from the camera. 


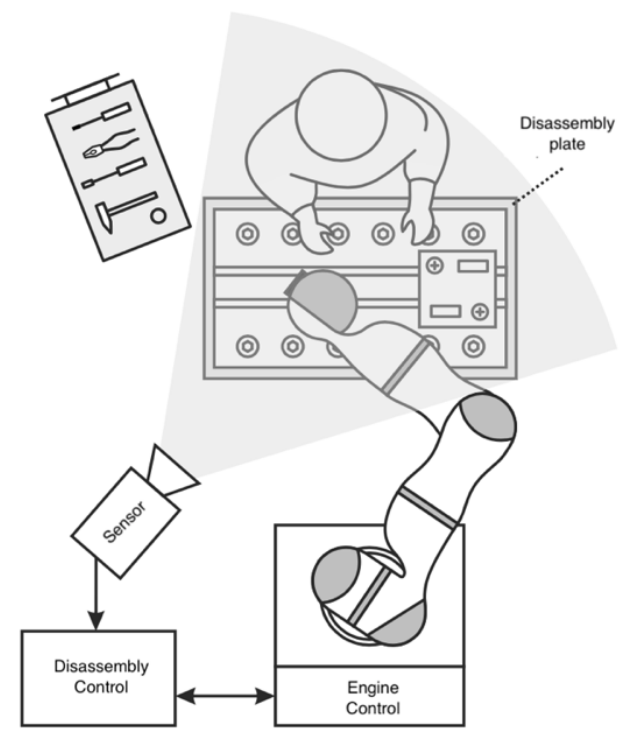

Figure 21: Disassembly work cell for EV battery disassembly, adapted from [10]

Figure 22 shows the experimental setup for EV battery disassembly set up by Wegener [10]. The disassembly robot can be controlled with a handheld device and is equipped with an automated screwdriver and gripper. The robot is placed directly on the working table which defines the area where the robot is allowed to move. On the working table a battery module of the Audi Q5 hybrid can be noticed as well as the discussed toolchanging station.

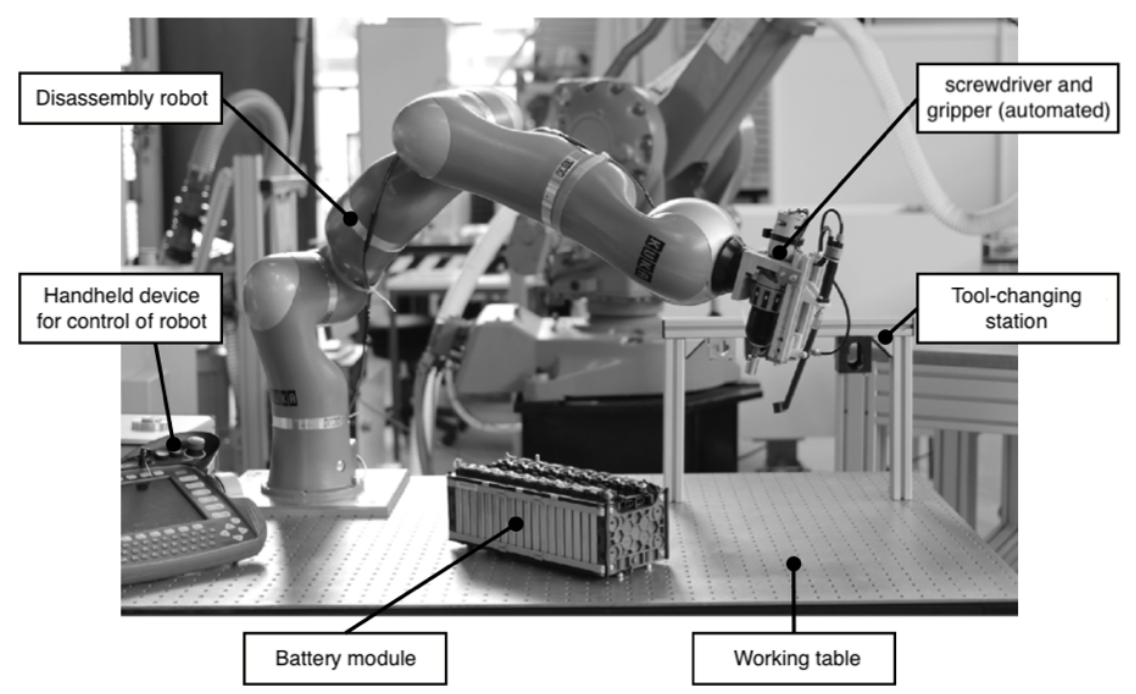

Figure 22: Working table with robot for EV battery disassembly, adapted from [10] 
Weigl-Seitz et al. [28] designed a work cell for disassembly of electronic devices. They stated that the layout of a disassembly cell should refer to known typical locations of parts and fasteners. As an example, the disks and drives of a PC are always located on the rear side of a PC, that makes it more easily to find them, if the right searching tool is located there. In most cases different grippers and sensors are necessary for disassembly of electronic goods. A second robot could be necessary for performing cutting tasks. Therefore, a disassembly system should be set up with several disassembly stations. It could be more efficient if there would be twice the amount of disassembly stations fulfilling time-consuming tasks. The outputs should collect scrap on the one hand but also valuable parts that could be re-used. Knowledge on the disassembled parts should be collected for more efficient disassembly. One possibility for that would be the identification of each goods serial number [28].

Scholz-Reiter, Scharke and Hucht [33] designed a robotic disassembly cell for TVs and monitors. The goal was to extract materials such as glass and plastic and to reduce the amount of landfill waste. The disassembly cell (see Figure 23) was equipped with extensive knowledge base and a system for image processing. It was designed for recycling more than 200 devices a day while it was stated that software and tool improvement could achieve a disassembly time of 2-5 minutes for each device. Starting at the input station the image processing system identified the model at first. Then, the disassembly robot worked on the disassembly with different tools such as a cutter or a screw loosening tool. A tool changing station was also provided. A second robot was installed for handling the recycled parts. It was equipped with different grippers as well as a tool changing station. This second robot sorted the disassembled parts to the conveyor 
for further processing. To handle screws on the rear side, another camera is mounted on the disassembly tool. The camera system also recognizes the position of the cable so that the harnesses can be cut by the cutting tool. Screws can be removed in a non-destructive fashion with a screwdriver or a destructive fashion by shearing of the screw head. An image processing system supervised the process and stored data for learning.

The software structure for the above system is illustrated in Figure 24. Different disassembly programs get generated with data from the product data base and the camera system. Furthermore, with current data from the camera system such programs can get adapted. The disassembly program generation triggers a dispatcher which in turn controls the machines in the system that includes the robot and conveyor.

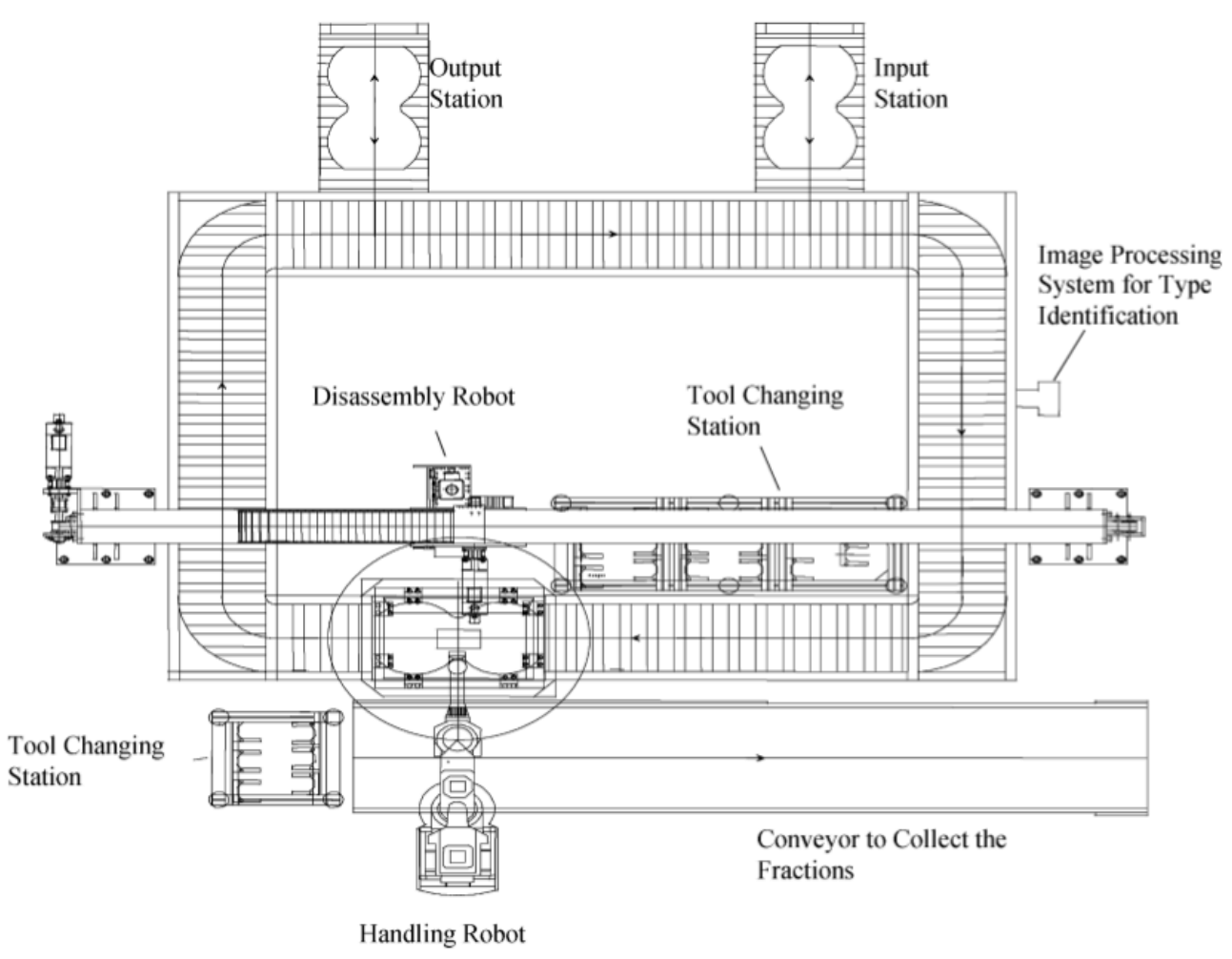

Figure 23: Layout of a disassembly cell for TVs and monitors [33] 


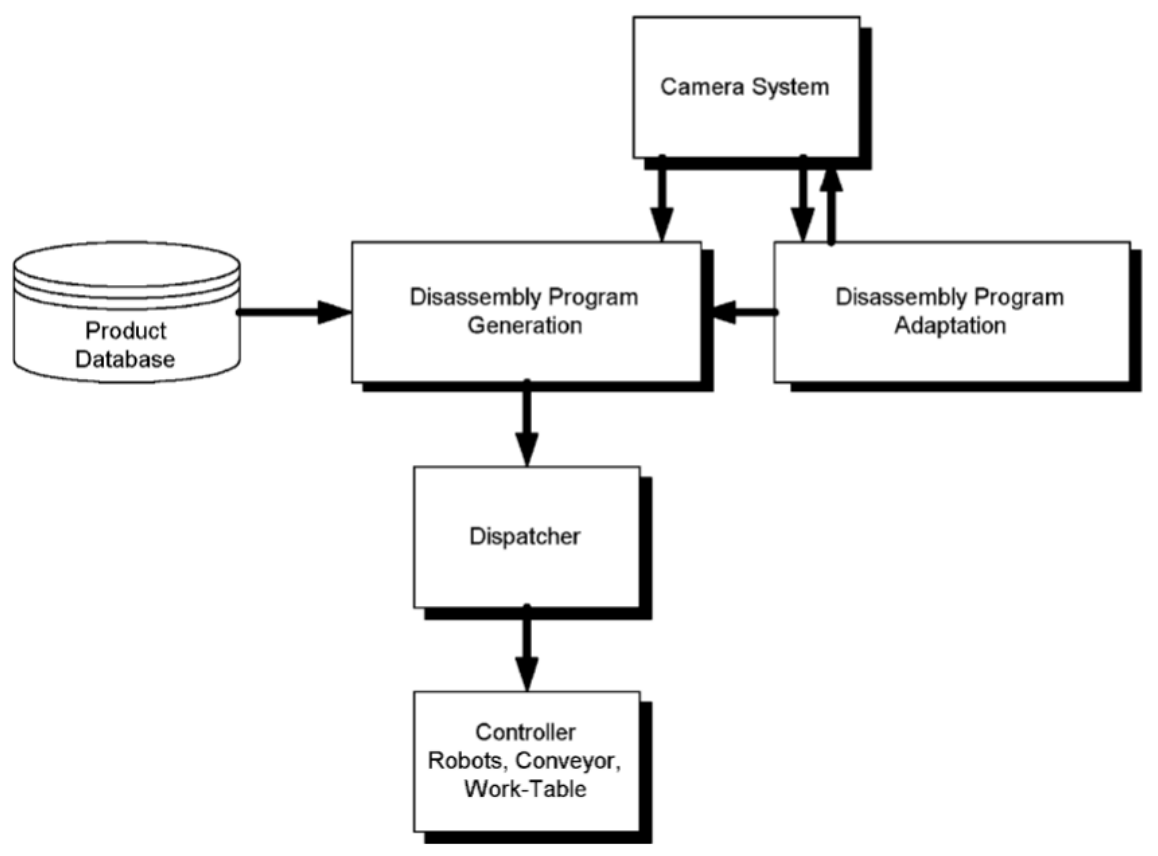

Figure 24: Software structure of a disassembly [33]

\subsection{Economics in Disassembly}

In setting up a system for automated disassembly of EV batteries a disassembly work-cell has to be designed, a vision system has to be developed and applied for finding parts and fasteners, and robotic solutions for different tasks have to be developed. At the same time, the disassembly of EV batteries must be economically justified and profitable. Different views on the economics of disassembly are summarized in this section.

Hermann et al. [60] assessed automation potentials for the disassembly of EV and hybrid vehicle batteries. As discussed in section (2.2.2) a criteria catalogue was developed that took technical (TAA) and economical (NAA) aspects into account. Twelve NA criteria were presented. Some are related to safety aspects such as the dealing with hazardous materials or cables carrying electrical current. From an economics point of view such operations create the need for expensive protective materials and special skilled 
workers. Those factors lead to higher costs and a lower profitability of manual disassembly but create a strong need for automated disassembly. Equipment costs are another listed factor in the economics of disassembly as well as the number of disassembly motions and the work-time that manual disassembly consumes resulting in high labor costs [60].

Thies et al. [2] examined the economics of automotive battery recycling with a model that has been used for comparing different scenarios. Those scenarios took different raw material prices, factor (e.g. electricity or wages) prices and different sales for BEV and PHEV/HEV vehicles on the European market into account. The high prices for the main raw materials were mentioned. Those materials include nickel, copper, aluminum, lithium carbonate and cobalt. It was approximated, that the raw material value of a $300 \mathrm{~kg}$ battery can be greater than $700 €$. Also, the cell chemistry has to be taken into account, because different cell chemistries could contain a much larger or smaller amount of valuable materials. The main costs include the high investments for machinery, wages and energy. It was stated, that all costs and revenues are very volatile and the prediction of the economic volume is difficult, even if the investments would only be justified for high volumes in recycling.

Hoyer [88] invented an optimization model for assessing the economics of automotive battery disassembly including different market scenarios. The model combines the available data with the objective function and constraints to make decisions. The input includes factors (e.g. the number of products or raw materials prices), the possible activities (e.g. capacities and investment costs) and other information (e.g. current market interest rate). The objective function is to maximize the net capital. The 
constraints include the maximum possible number of recyclable products. As the result, it can be planned, how many recycling stations should be operated.

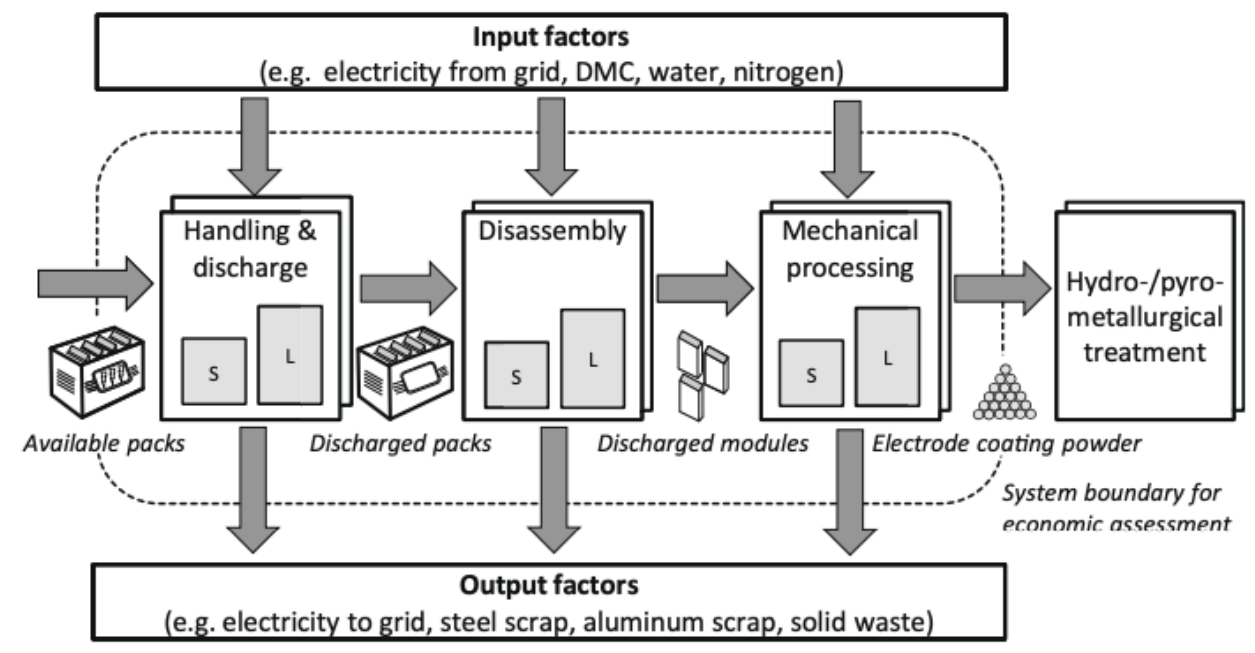

Figure 25: Model for assessing the economics of disassembly [2]

The model of Hoyer [88] was adjusted by Thies et al. [2]. Figure 25 shows the model. Input factors include mainly energy and water, while electrical energy is also an output at the moment when the batteries are discharged. Other outputs are metals and waste. Additionally, the available battery packs enter the system boundary, get discharged, disassembled to modules and processed mechanically to produce electrode coating powder. This powder leaves the system and is treated hydro- and pyrometallurgically in another step (and facility) for regaining the basic raw materials.

In order to use the model for economic analysis, data on equipment and material prices were collected and scenarios on future scales of BEV and hybrid vehicle batteries were developed. Three scenarios for the markets of electric vehicles were presented that considered an optimistic, realistic and pessimistic market future. Those scenarios included predictions for the stock of BEV, PHEV and HEV in Europe from 2015 until 2020. The 
distinction is important because BEV batteries are much larger and heavier than PHEV and HEV batteries. Therefore, more valuable material can be gained from one battery, but disassembly is also more complicated. Furthermore, it is difficult to predict the span of useful life for a battery because it is highly depended on the usage, but a "second life" was considered which would shift disassembly to a later point in time. The weights of the different battery types were normalized to BEV battery equivalents. Typical factory parameters such as working times, maintenance times and market share were predicted. The realistic scenario assumed 51,500 BEV battery equivalents for 2025 , which would allow large scale industrial disassembly. Investment costs and operating expenses for each disassembly step were listed. Additionally, the costs and revenues for all input- and output- factors (see Figure 25) were shown with three scenarios [2].

The model from Thies [2] has been applied for a realistic EV stock and price scenario. Break-even analysis resulted in a payback period of 5 years. That relatively short payback period underlines the economic prospects of EV battery recycling. Breakeven analysis has been performed for different additional investments such as electricity recuperation (feeding discharged electricity back to the grid).

Harper et al. [11] described, that the use of disposed EV batteries as energy storage is one first possibility of a "second life" before recycling. Especially due to the rise of fluctuating energy supply of clean renewables or in areas with generally weak grids energy storage would be very useful for grid stabilization. But even after a "second life" recycling and disassembly are necessary. It was stated that the batteries should be disassembled at least to the level of battery modules. A comparison of pyrometallurgy, hydrometallurgy and direct recycling as different ways of material treatment was reported 
They concluded that direct recycling would provide the best quality of recovered material, while pyrometallurgy is much cheaper.

Wegener [10] described that sorting of battery designs and types could reduce costs due to learning effects and a possibly higher degree of automation. Gerbers et al. [86] stated that a high level in the future of automation in EV disassembly is necessary for economic performance. Therefore, it is necessary to increase the reliability and lower the costs of hardware and software for automated workstations. It was mentioned that investment costs for human-robot workstations are very high and there are no standardized applications, so cost-intensive individual development is required. One possibility would be to standardize interfaces for robotic tools. Integrated sensors could also lower the complexity of the tools and save costs [86]. A further description of those simplified tools was provided by Gerbers et al. [89].

Li et al. [23] investigated selective disassembly that prioritizes economically feasible operations in the field of electronic equipment. Decision models were developed that included many criteria and adaptive decisions. In general, operations should be prioritized by the material value. Duflou et al. [90] performed data mining on the economics of disassembly based on different cases of full- or partial disassembly such as fridge recycling in China. The profitability's of the cases were compared. They stated, that automated disassembly improves process reliability, but more disassembly friendly design is also needed. A technical analysis combined with a market analysis for EV batteries was performed by Mahmood and Gutteridge [91]. They also discussed the environmental impact of EV battery recycling and the benefits of a "second life" after the battery efficiency becomes too low for usage in EVs. Kampker et al [92] discussed the 
impacts of a "second life" and the remanufacturing on the economics and the environmental impact of EV batteries and described how a circular economy for the raw materials of EV batteries can be established. Mathews et al. [93] explained how EV batteries can be used in a second life as an energy storage for solar power plants. Cong, Zhao and Sutherland [94] described how economic value at the EOL can be created with non-destructive disassembly operations. White, Thompson and Swan [95] described how frequency regulation in the electricity grid can be improved with the usage of EV batteries in their second life.

\subsection{Discussion}

Summarizing the presented studies, there have been many studies on automating disassembly of different products, mostly electronic waste. There have been also some investigations on achieving automated disassembly for EV batteries, but a disassembly cell has only been presented for the smaller HEV/PHEV batteries, while the automated disassembly of large and heavy EV batteries faces the same and some additional technical challenges as such as the additional cooling system, higher weights and the size. The discussed approaches for the automated disassembly of electronic waste can partly be applied to the disassembly of EV batteries. The most important results from previous studies will be pointed and related to the challenges of automated EV battery disassembly. The structuring of parts in EV battery could be a first approach for developing disassembly strategies or assessing which tools will be needed. In a later stage the disassembly sequences could be optimized. From parts and fasteners hierarchy it could also be determined, which parts have to be disassembled for reaching the most valuable parts. 
EV batteries come in many different sizes and shapes and the design differs strongly by the manufacturers but the main parts such as battery cells, battery modules and harnesses appear in every design. Additionally, it would be difficult to acquire CAD data with exact positions of parts and fasteners for every model, fasteners appear in different directions or can be hidden, and damaged or soiled parts could also be present. Without more standard designs, it is difficult to construct a disassembly system that can handle different types of batteries. Those facts strongly recommend the use of humanrobot collaboration. In that concept, the robot performs repetitive task or the handling of heavy weights, while the human worker solves unpredictable problems and disassembles parts that are not reachable or difficult for the robot to do. The concept of human-robot collaboration was applied in the study on the automated disassembly of Audi Q5 hybrid battery. The used LWR was very suitable for human-robot collaboration, because its light weight is safe for the human and decreases the risk of injuries. For the disassembly of the 2017 Chevrolet Bolt which is an example of a BEV much heavier parts have to be carried than for HEV and PHEV batteries. Each Battery Section of the 2017 Chevrolet Bolt weighs more than $50 \mathrm{~kg}$ [22], while LWRs are suited to carry low weights, typically below $10 \mathrm{~kg}[10]$. Because of that either the heavy lifting tasks have to be performed with a manual crane as done by Kelly [21], or more powerful robots have to be employed, which would lead to more challenges for human-robot collaboration.

Approaches on the main tasks needed for automated EV disassembly from different studies have been reviewed. Those include parts identification using vision systems, unscrewing operations, tool changing operations, grabbing operations, prying and cutting operations, and work-cell layout. 
Vision systems should detect and localize parts and fasteners, so that the automated tool can approach and disassemble those. Automated vision-based detection systems are preferable to a human teaching fastener positions since it does not require teaching for different batteries. However, lighting conditions and the conditions of the battery make it difficult to identify fastener positions with high accuracy. Also, studies on other electronic waste showed the current difficulties of screw detection. Recent approaches using deep neural networks to identify fasteners are promising. The creation of a model of the environment or structure of the parts and fasteners can be done from photos taken from the vision systems. Some typical parts such as cables can be identified by color or shape and the help of heuristics and algorithms. Another approach is the identifying of the serial number of an EV battery, or even of individual parts and creating a database of known models and disassembly approaches and fastener or part positions for those. Future research should improve the accuracy of fastener position location. For complex products with different fasteners vision system should be able to detect the type and size of a fastener in order to let the bit changing tool prepare the end-effector. Making Using neural networks for the learning of the vision system. Furthermore, the labeling of products and parts with RFID tags or QR-codes combined with an international data exchange in a cloud will make it possible to know the locations and types of parts and fasteners in advanced if a product once was disassembled.

Automated unscrewing tools use the identified fastener positions and approach and align with the fastener with the help of search algorithms such as the spiral search. Force sensors were applied for detecting the alignment and if a fastener is finally loosened. Also, destructive boring out of screws would be possible, for example if screws are 
corroded. In order to deal with different sizes and kinds of fasteners, a bit changing system is necessary. If multiple robots are used in a work-cell, each robot and the human worker should have their own tools and tool- or bit-changing facilities. Future research should improve the reliability of the unscrewing tools and provide a fast bit changing. Simultaneously product design must shift to a disassembly friendly design in an order that fasteners will be easily accessible for automated screwdrivers.

Followed by the loosening of fasteners a grabbing of the disconnected parts has to take place. Different approaches for grabbing tools have been presented. These include simple two- or three- finger grippers or specialized tools for certain operations such as lifting tools for heavy parts (e.g. Battery Modules). One gripper system was developed for taking out a single battery cells from EV battery modules and simultaneously measuring their state of charge. The disassembly of a BEV battery needs flexible and different grabbing tools, due to the large variety of shapes and weights of the parts. In the case of a large battery, such as 2017 Chevrolet Bolt battery, there are many covers that need to opened with a prying tool. In that special case, the covers just need to be opened in order to reach the nuts below. In a later step the covers are disassembled together with large busbars by grabbing. Future research should focus on more reliable grabbing tools that can even reach into tiny gaps or lift heavy parts. A large variety of grabbing operations needs either a large variety of grabbing tools or the development of some multifunctional ones.

Almost all EV batteries have cables that are held by many clips. The nondestructive disassembly of such connections is very challenging. Thus, the employment of a cutting tool is needed. One concept for such a cutting tool was presented that was a 
high-speed rotatory tool. For many cutting operations, it could be useful to ensure a collaboration of two robots to perform this task. Depending on the difficulty of the cutting task it could also be more suitable for the human worker in human-robot collaboration arrangement. There is a lot of potential for research on flexible and reliable cutting tools for cables.

The disassembly work cell layout for the disassembly of HEV/PHEV batteries was described as a working table where LWR and human worker are placed opposite of each other. Another proposed layout for e-waste was composed of a disassembly line with multiple stations for single tasks. That concept promised higher quantities and a second robot was employed for material handling to collect the materials efficiently. The proposed layout with one LWR opposite to the human would not be suitable for the large and heavy EV batteries. One LWR would not be able to reach all parts of the battery. Furthermore, a single LWR would not be able to lift large and heavy parts such as cooling plates or battery modules. Also, the handling of the large and heavy batteries and large sizes and the variety of disassembled parts creates further challenges. The high number of disassembly steps combined with the inconsistency in the design of EV batteries suggests that a line disassembly configuration won't be suitable also. This area needs future research. One study could be the comparison of different workstation configurations, for example a layout with one large gantry robot compared to multiple smaller LWR robots.

Investigations towards the economics of disassembly of EV batteries were summarized. Different scenarios for the prices of recovered raw materials and the amount of disposed EV batteries were summarized. For realistic predictions and scenarios, the amount of disposed EV batteries will be high enough for economically feasible 
disassembly. Furthermore, future standards such as common types of fasteners or similar sizes of the battery cells and modules in the design of EV batteries will simplify the disassembly and raise economic benefits. Those economic investigations should be performed again with new predictions and data on disposed EV batteries in future. Additionally, economic optimization of single workstations can be performed. 


\section{Analysis and Assessment of EV Battery Recycling}

A BEV battery has been investigated in details in this study. At first a list of all parts and fasteners was created, and the relations between the parts and fasteners have also been examined. A structure was developed that shows all part connections. Based on that one possible disassembly sequence was developed. Following that an assessment on the technical possibilities and the economic needs for disassembly of each single disassembly step took place.

\subsection{Structuring of Parts and Disassembly steps}

The structuring of the parts was developed using as an example the 2017 Chevrolet Bolt battery. The material is based on the disassembly and reassembly video of Kelly [21]-[22]. In the first video [21] the EV battery has been manually disassembled down to the level of the battery modules. The parts that remained in the tray after extracting the modules have also been disassembled.

\subsubsection{Identification of Parts and Fasteners}

For the structuring of the parts, we differentiated between parts (numbered: $\mathrm{P \# )}$ and fasteners (numbered $\mathrm{F} \#$ ). Based on the videos [21]-[22] all parts and fasteners have been identified that haven been taken apart from the battery tray. The battery modules have not been disassembled further. In total, 76 parts and 374 fasteners have been identified and labeled. Table 1 shows an example of labeled fasteners. The IDs F1-50 correspond to the 50 bolts around the top cover (additionally there are 6 bolts on the upper part of the cover), those are further described, and an image gives an overview about the location. Typical identified fasteners are bolts, nuts and screws but clips or covers are also 
labeled as fasteners if they have to get opened or unclipped. There is a large amount of different parts with a large variety in shape and size. Most typical parts are brackets, covers and busbars due to the design of an EV battery. As described in section 2.2.1 the parts and fasteners can also be labeled as active and passive parts [46]. In the example of the EV battery the passive parts "Top Cover (P1)" and "Battery Tray (P76)" are connected by the active parts "Bolts (F1-56)". So, the active parts connecting two passive parts have to get disassembled first, before the passive parts can be taken.

Table 1: Description and image of a fastener

\begin{tabular}{|l|l|l|l|l|}
\hline ID & Name & $\begin{array}{l}\text { Descripti } \\
\text { on }\end{array}$ & Image \\
\hline F1-50 & $\begin{array}{l}\text { Bolts 1- } \\
50\end{array}$ & $\begin{array}{l}\text { Bolts for } \\
\text { the top } \\
\text { cover (P1) } \\
\text { to Battery } \\
\text { Tray (P81) }\end{array}$ & & \\
\hline
\end{tabular}

\subsubsection{Development of a Disassembly Graph}

In the next step, a disassembly graph was developed. For every part and fastener, it was verified, which parts or fasteners had to get disassembled first. As discussed before the "Top Cover" can be taken apart, if the 56 bolts connecting it to the battery tray have been unfastened. Figures 25-27 show the developed disassembly graph. The parts are illustrated with grey boxes and the fasteners with blue boxes. All boxes with a dashed fringe are accessible/visible before starting the disassembly. The orange boxes are used as continuations $(\mathrm{C \#})$. The graph shows the direct predecessors and successors for each individual fastener (or group of fasteners) and part. The presented graph is a similar to the "graph model of the environment" presented by Hohm, Müller Hofstede and Tolle [46], but it clearly points out which parts are passive (P\#) or active (F\#). Furthermore, the arcs 


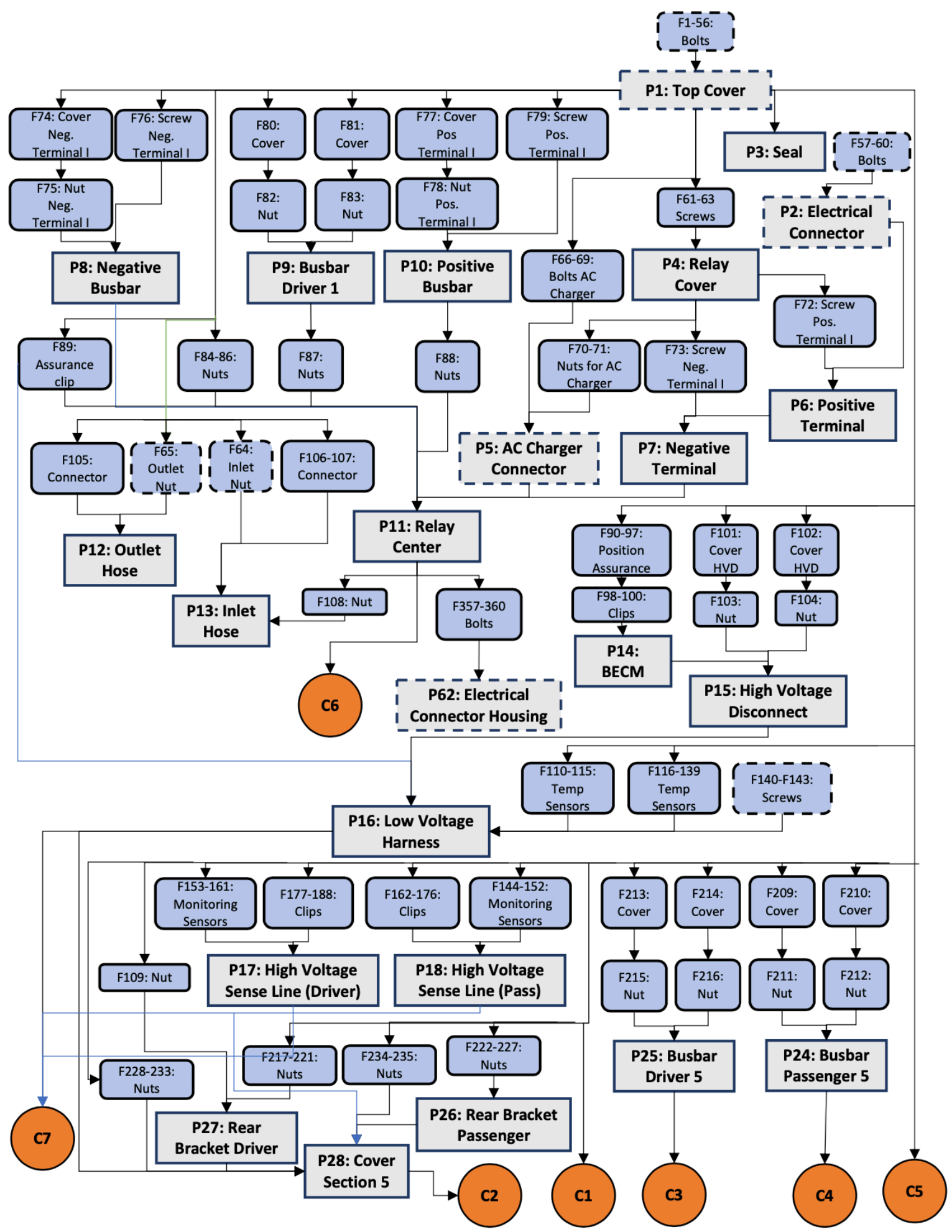

Figure 26: Disassembly graph (page 1) 


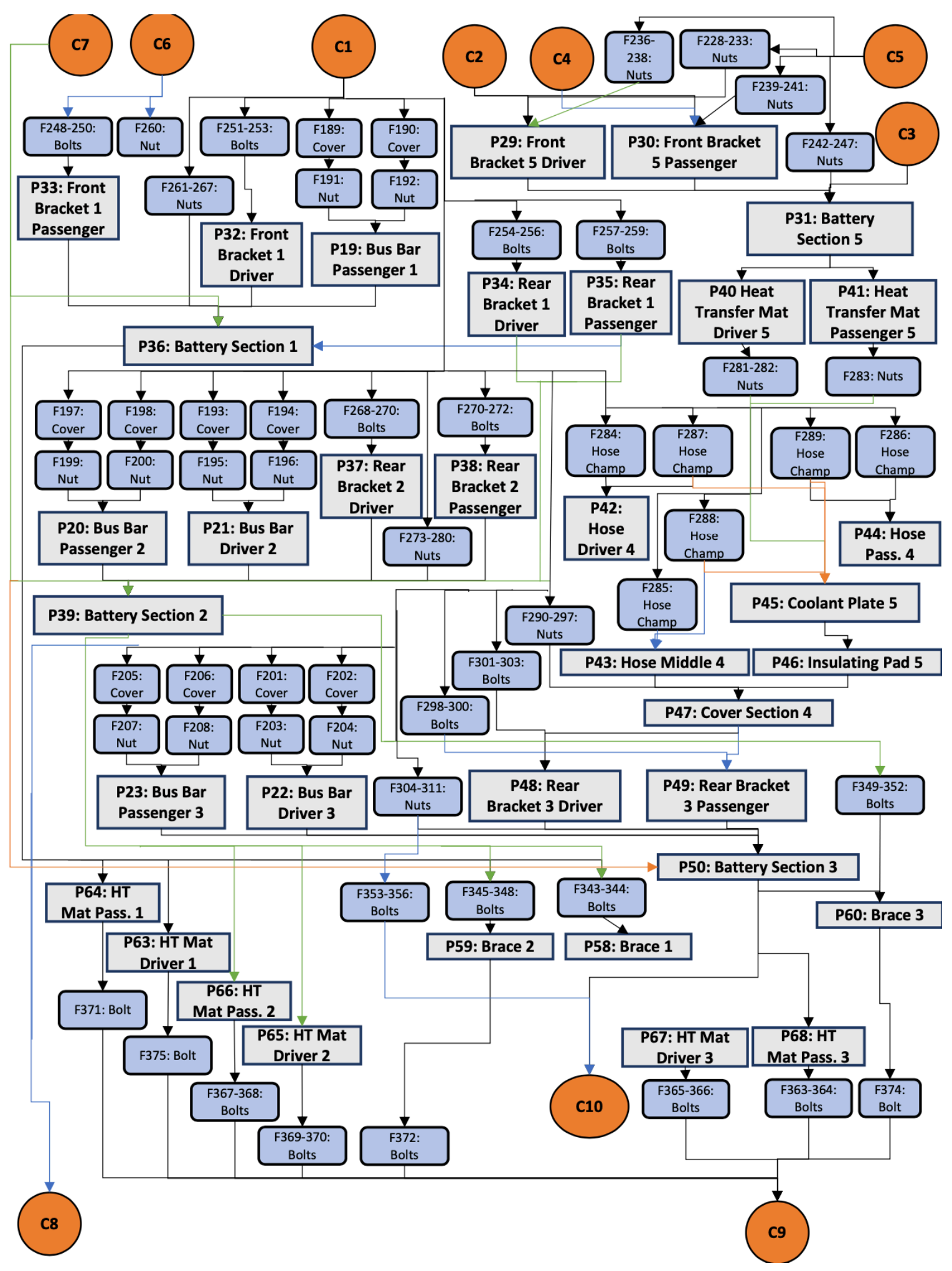

Figure 27: Disassembly graph (page 2) 


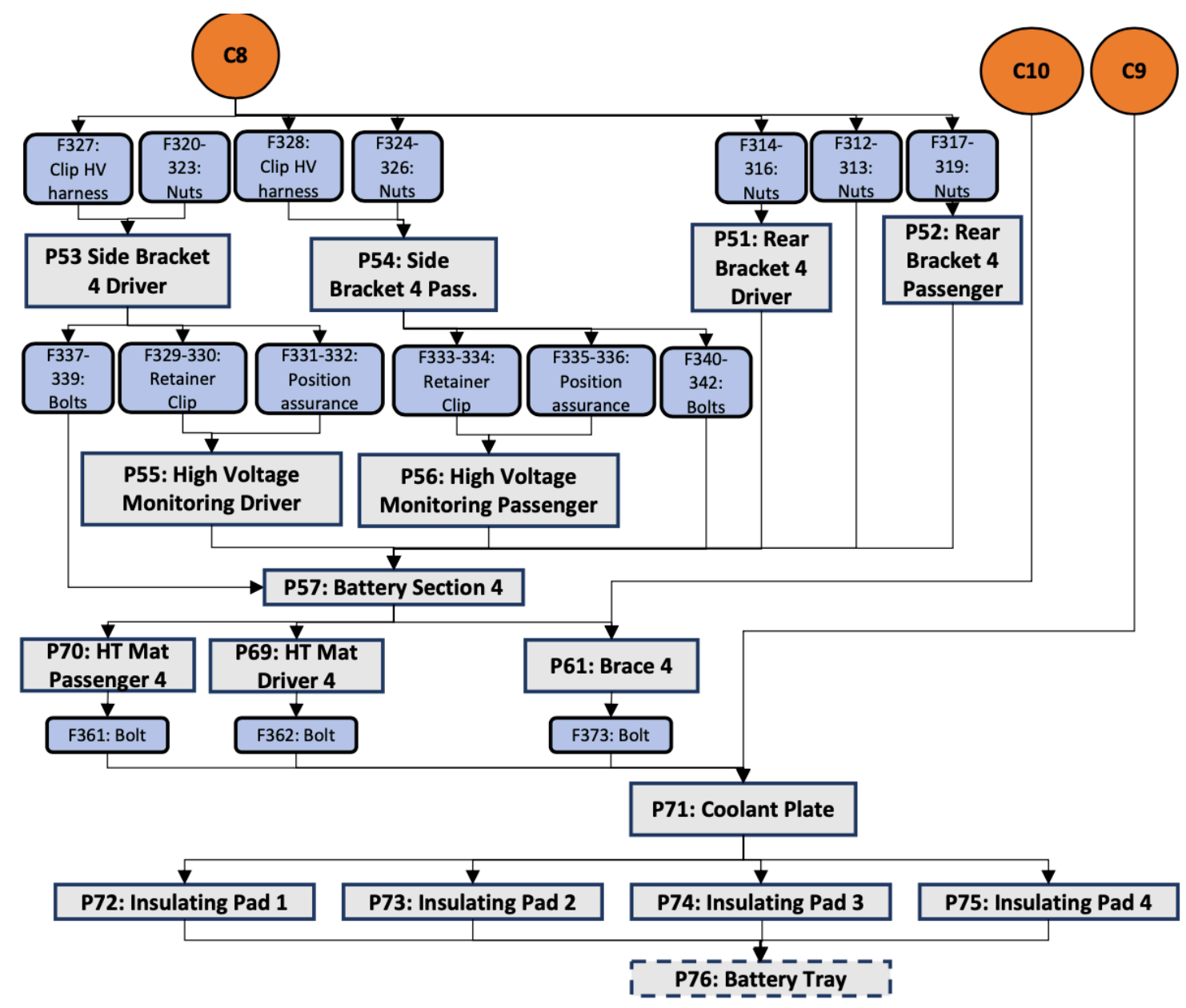

Figure 28: Disassembly graph (page 3)

show the direction of disassembly. The arc structure for a disassembly graph was presented by Li et al. [23].The presented graph combines both approaches. Active and passive parts (fasteners and parts) are distinguished, but also a disassembly direction is shown.

\subsubsection{Suggested Disassembly Sequence}

Although there are several approaches for finding an optimized disassembly sequence with the help of Operations Research algorithms, it was decided to just find one exemplarily, manually optimized disassembly sequence. For that purpose, based on the 
disassembly graph, similar parts or fasteners that could be disassembled in one step have been combined, thus the number of disassembly steps was significantly reduced.

Table 2 shows partial listening of the 46 disassembly steps. The steps are numerated with the ID "D\#." Furthermore, the parts or fasteners of each disassembly step are listed. The next column shows the quantity of the different parts or fasteners. That helps for later assessment to find the number of necessary tool changes. The necessary tools are also listed. Those can be compared to previous automation approaches for electric and hybrid vehicle batteries [6], [58]-[60], [63]. The comments column is filled with further information or predicted difficulties. The size of parts, or general working space to approach those are listed und the "Approximate Size" column. The last column just lists a first estimation about the difficulty of automation for that part that could be used later in assessments.

Table 2: Examples of disassembly step descriptions

\begin{tabular}{|c|c|c|c|c|c|c|c|}
\hline \multirow[b]{2}{*}{$\begin{array}{c}\text { Step } \\
\#\end{array}$} & \multicolumn{7}{|c|}{ Details } \\
\hline & $\begin{array}{l}\text { Parts and } \\
\text { Fasteners }\end{array}$ & $\begin{array}{l}\text { Connector type } \\
\text { and quantity, or } \\
\text { removable part }\end{array}$ & Access & Tools & Comments & $\begin{array}{l}\text { Approx. } \\
\text { Size }\end{array}$ & $\begin{array}{l}\text { Automation } \\
\text { Difficulty? } \\
\text { Easy/Difficult// } \\
\text { Challenging } \\
\text { (First } \\
\text { estimation) }\end{array}$ \\
\hline D1 & F1-56 & 56 hexagonal Bolts, & Top, open & Screwdriver for bolts & $\begin{array}{l}6 \text { bolts around service plug connector } \\
\text { (higher in z-direction) } \\
\text { Difficulties due to large area. }\end{array}$ & $\begin{array}{l}2 \mathrm{~m} \times 1 \mathrm{~m} \\
\mathrm{x} 20 \mathrm{~cm}\end{array}$ & Easy \\
\hline D6 & $\begin{array}{l}\text { F74, F77, } \\
\text { F80-81 }\end{array}$ & 4 Covers & Top & $\begin{array}{l}\text { Prying tool for opening } \\
\text { covers }\end{array}$ & $\begin{array}{l}\text { Need for a prying tool, that can open } \\
\text { covers }\end{array}$ & $50 \times 50 \mathrm{~cm}$ & Difficult \\
\hline D12 & F64-65 & 2 Large Nuts & Side, inside & $\begin{array}{l}\text { Nutrunner, } \\
\text { Screwdriver, to hold } \\
\text { Nuts from the inside }\end{array}$ & $\begin{array}{l}\text { Hold it fixed from inside and unscrew } \\
\text { nut from outside, very difficult to } \\
\text { automate, large screwdriver needed, } \\
\text { grabbing would also be difficult. }\end{array}$ & $\begin{array}{l}10 \times 10 \times \\
10 \mathrm{~cm}\end{array}$ & Challenging \\
\hline D19 & $\begin{array}{l}\text { F103-104, } \\
\text { F109, } \\
\text { F140-143, } \\
\text { F191-192, } \\
\text { F195-196, } \\
\text { F199-200, } \\
\text { F203-204, } \\
\text { F207-208, } \\
\text { F211-212, } \\
\text { F215-280, } \\
\text { F290-326 }\end{array}$ & $\begin{array}{l}88 \text { Nuts, } \\
24 \text { Bolts, } \\
4 \text { Screws }\end{array}$ & $\begin{array}{l}\text { Top (some are } \\
\text { partly hidden) }\end{array}$ & Screwdriver/ Nutrunner & $\begin{array}{l}\text { Tool change needed, some of the nuts } \\
\text { and bolts are more difficult to detect, } \\
\text { because those are below brackets or in } \\
\text { between the sections at the bottom. } \\
\text { Extended screwdriver/ nutrunner needed }\end{array}$ & $\begin{array}{l}180 \times 90 \\
\times 40 \mathrm{~cm}\end{array}$ & Easy \\
\hline D39 & $\begin{array}{l}\text { P36, P39, } \\
\text { P50, P57 }\end{array}$ & 4 Battery Sections & Top & $\begin{array}{l}\text { Battery Lifting Tool for } \\
\text { Battery Sections }\end{array}$ & $\begin{array}{l}\text { Special lifting tool needed, lifter must } \\
\text { get adjusted on the battery, slow lifting } \\
\text { encouraged. Balance whole EV battery } \\
\text { while lifting. }\end{array}$ & $\begin{array}{l}40 \times 80 \\
\times 15 \mathrm{~cm}\end{array}$ & Difficult \\
\hline
\end{tabular}


The D1 "Bolts for Top Cover" is the first disassembly step and describes the unfastening of the 56 hexagonal bolts around the top cover and service plug connector. Besides the bolts for the electrical connector (see Figure 26), it is the only possibility to start the disassembly process. Figure 29 shows the top cover. The access is open and from the top, which simplifies detection. The disassembly tool for this step is a screwdriver for the bolts which is necessary. It is commented that the work area is large. This means that more than one robot or one large robot may be needed to perform the task. Additionally, an approximation for the size of the work area is given as one meter in width and two meters in length. The 6 bolts around the service plug connector are $20 \mathrm{~cm}$ higher. That has to be borne in mind for detection and access. The first estimation suggests an easy automation of disassembly. There are several choices for automated screwdrivers and the access and detection seem feasible.

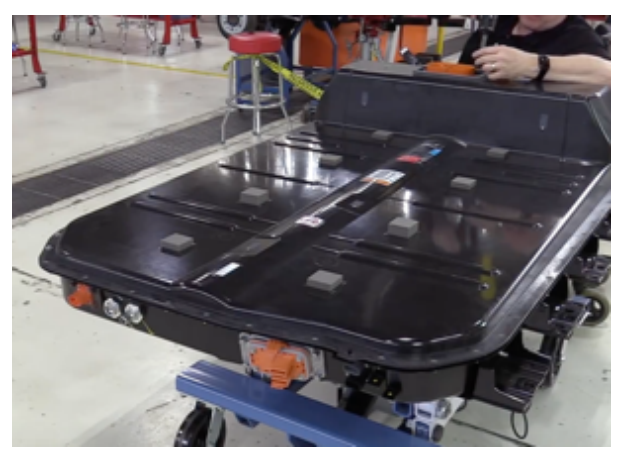

Figure 29: Disassembly step D1, the Top Cover [21]

An example for the combination of different disassembly steps is D6 "Covers for Busbars (Front)." The four covers (F74, F77, F80, F81), shown in Figure 30 could be opened in one step, because those are similar covers and they are also located in the same area of the EV battery. They are accessible from the top and a prying tool is needed for opening. A first estimation implies that automation is difficult because of the difficulty of 
the prying process. Furthermore, it could be hard for a vision system to find the spot for placing the prying tool.

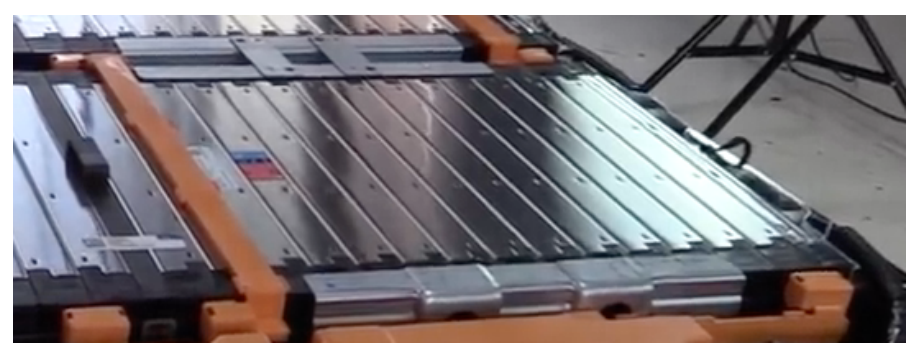

Figure 30: Disassembly step D6, the four covers for the busbars in the front [21]

Disassembly step D12 “Big Nuts for Coolant Hoses” (see Figure 31) represents an example of a challenging step. It is unique and needs special tools because of the size of the nuts. The access from the side which is also very difficult. From that first estimation D12 has a very low automation potential.

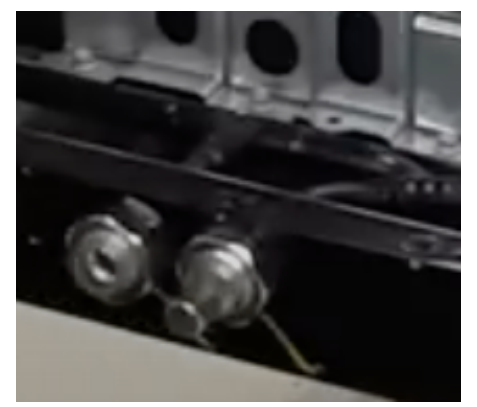

Figure 31: Disassembly step D12, the Big Nuts for Coolant Hoses [21]

An example where a lot of fasteners have been combined is D19: "Nuts, Bolts and Screws for Busbars and Brackets, High Voltage Disconnect and Battery Sections." That disassembly step combines the unfastening of 88 nuts, 24 bolts and 4 screws, while only a few tool changes are necessary, and the operations are very similar and repetitive. Figure 32 shows an example of such screws. Most of the fasteners are relatively easy to detect and approach, while some are partly hidden below brackets. Therefore, an extended 
screwdriver or nutrunner could be applied. The workspace is very large, because the fasteners are spread over the whole battery. Finally, that step is considered as easy because of the high automation potential of unscrewing tools.

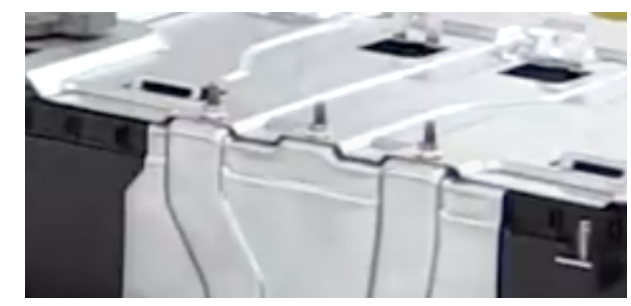

Figure 32: Example for screws in disassembly step D19 [21]

D39 "Battery Sections 1-4 Lifting" is one of the most important steps. Four of the five Battery Sections are separated from the remaining battery parts. Figure 33 shows the lifting of one of the Battery Sections. While this part is large and relatively easy to detect, it is more difficult to find the spots on the part to place the lifting tool. The handling of heavy parts is another challenge, so a special lifting tool or crane is needed. Due to the glued heat transfer mats below the Battery Section the lifting must also be down slowly. Also, the lifting tool has to be adjusted to balance the Sections while lifting. Therefore, this step is ranked as difficult.

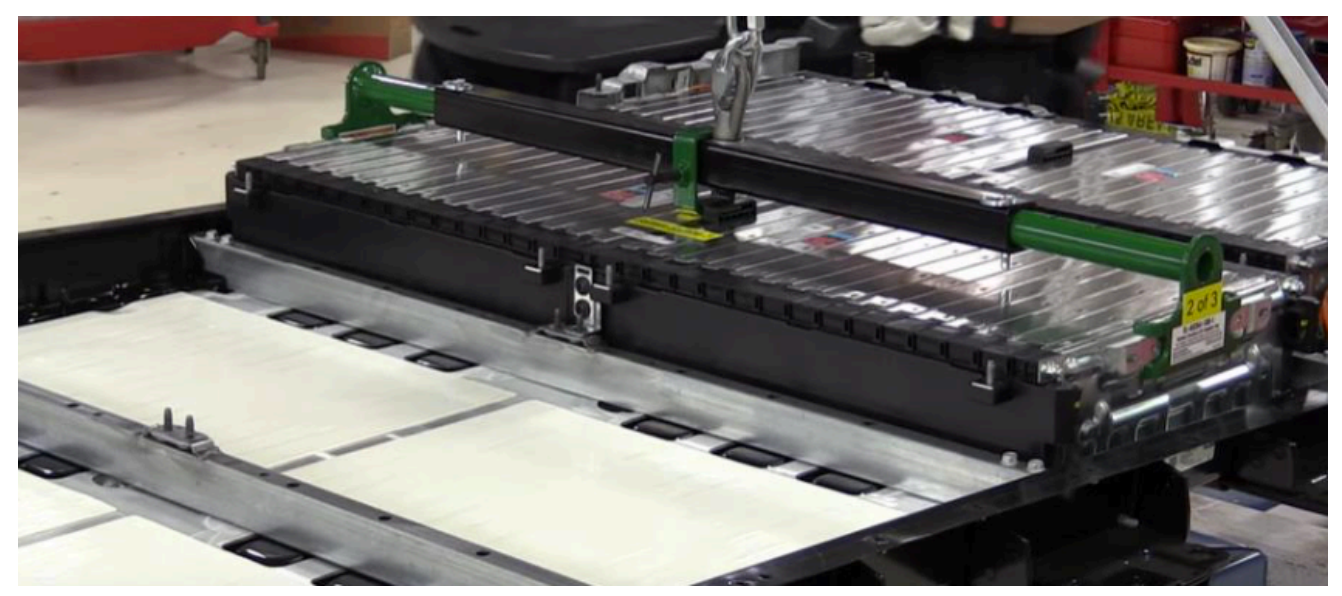

Figure 33: Disassembly step D39, the lifting of four battery modules [21] 


\subsection{Assessment of the Automation Potential of Disassembly steps}

Based on the developed 46 disassembly steps (see section 3.1.3) and the collected information about each single step, an assessment of automation potential for each step has been realized. A criteria catalogue was created for this task. The catalogue provides criteria for the technical possibilities and the economical necessities for automation. This catalogue was applied to an example of one PHEV and one BEV battery. The results for the hybrid vehicle and EV battery have been compared to results from literature, and the differences and similarities are discussed.

\subsubsection{Criteria Catalogue for step Assessment}

Herrmann et al. [60] proposed a catalogue with 17 criteria for the assessment of hybrid vehicle and EV batteries. Some similar criteria have been aggregated to get a simpler catalogue with 10 criteria. The criteria are 5 each for the technical assessment (TAA) and for the economical assessment (NA). In comparison to [60], each criterion was assigned the same weighing factor.

Table 3 shows the list of criteria. For each criterion a scoring between -2 and 2 is possible. With a weighing factor of 10 , the range of possible scorings is from -100 to 100 for each NA and TAA. For the economical assessment the time a human worker needs are the most important aspect. The first two criteria (NA1 and NA2) account for that time. The number of motions is relatively easy to count. For the disassembly time, approximations have been made on the basis of the Methods-Time Measurements (MTM) [96]. This technique is well used in industrial settings, where standard times for certain movements are fixed. Kroll and Hanft [34] applied that method on disassembly tasks for 
electronic devices. They combined that approach with guidelines for the Design for disassembly (DFD) [64], and they provided examples of times for different tasks.

Table 3: Assessment criteria

\begin{tabular}{|l|l|l|}
\hline & $\#$ & Criteria \\
\hline & 1 & Number of Motions (human) \\
\hline & 2 & Duration of manual disassembly time in seconds \\
\cline { 2 - 3 } & 3 & Danger (High voltage protection, hazardous materials) \\
\hline & 4 & Weight \\
\hline & 5 & Priority (value) \\
\hline & 1 & Complexity of motion (for robot, number of different motions) \\
\hline 2 & 2 & Access for end effector \\
\hline 3 & Possible detection \\
\hline 4 & Automation potential for robotic end effector \\
\hline 5 & Material handling \\
\hline
\end{tabular}

Danger for the human worker (NA3) is another criterion where the dangers ranges from sharp edges and chemicals to the danger of high voltage which is present in EV batteries. The necessary protection for a human worker and its costs and longer working times have to be considered.

The weight of a part (NA4) is another important factor. Due to health considerations, human workers cannot handle heavy weights for a long time or perform many repetitions. Steinberg and Windberg [97] documented weights and posture criteria such as bending and twisting and rankings have been given. For the disassembly assessment we used a combination of both, posture and weights. The documented time criterion has not been taken into account, because it is already included in the time criterion discussed earlier. Table 4 shows the scorings for the weight criterion. High 
weights or much bending tend to a preference for automation, while low weights would suggest manual disassembly.

Table 4: Criteria scorings on part weights and ergonomics (NA4)

\begin{tabular}{|l|l|l|l|l|}
\hline $\mathbf{2}$ & $\mathbf{1}$ & $\mathbf{0}$ & $\mathbf{- 1}$ & $\mathbf{- 2}$ \\
\hline $\begin{array}{l}\geq 25 \mathrm{~kg} \text { or strong } \\
\text { bending/twisting and } \\
\text { weight far away from } \\
\text { body }\end{array}$ & $\begin{array}{l}<25 \mathrm{~kg} \text {, or far away } \\
\text { or strong bending/ } \\
\text { twisting }\end{array}$ & $\begin{array}{l}<15 \mathrm{~kg} \text { or medium } \\
\text { bending of body, } \\
\text { part far away from } \\
\text { body }\end{array}$ & $\begin{array}{l}<10 \mathrm{~kg} \text { or } \\
\text { little } \\
\text { bending of } \\
\text { body }\end{array}$ & $\begin{array}{l}<5 \mathrm{~kg} \text {, straight } \\
\text { upper body, } \\
\text { part near to } \\
\text { body }\end{array}$ \\
\hline
\end{tabular}

The scoring values for the priority criteria (NA5) are shown in Table 5. As discussed before, the modules and cells contain a high amount of valuable materials. Additionally, there are valuable materials in other parts or recyclable expensive components. Examples can be large pure aluminum parts or the BECM as a more expensive recyclable part. So, the highest rating (two) will be given if the step separates a valuable recyclable part and is necessary to reach the modules. A scoring of one is given if it is a necessary step in order to achieve access to the battery modules, while zero corresponds to just relatively valuable parts. If at least a sorting of different materials takes place it will score minus one. A scoring of minus two will be given if just unrecyclable low-cost parts are removed. In summary, NA5 this is the most economically driven criterion.

Table 5: Criteria scorings on the priority for disassembly (NA5)

\begin{tabular}{|l|l|l|l|l|}
\hline $\mathbf{2}$ & $\mathbf{1}$ & $\mathbf{0}$ & $\mathbf{- 1}$ & $\mathbf{- 2}$ \\
\hline $\begin{array}{l}\text { Necessary in order to } \\
\text { reach cells and other } \\
\text { valuable materials }\end{array}$ & $\begin{array}{l}\text { Necessary in } \\
\text { order to reach } \\
\text { cells }\end{array}$ & $\begin{array}{l}\text { Not necessary to } \\
\text { reach cells, but } \\
\text { other valuable } \\
\text { materials }\end{array}$ & $\begin{array}{l}\text { Not very valuable } \\
\text { materials but } \\
\text { sorting different } \\
\text { materials for further } \\
\text { recycling }\end{array}$ & $\begin{array}{l}\text { Low cost } \\
\text { materials, not } \\
\text { necessary for } \\
\text { cells }\end{array}$ \\
\hline
\end{tabular}


The first TAA criterion (TAA1) assesses the complexity of the robotic motion. The number and difficulty of the motions are combined. Standard movements such as translational or rotational movements are seen as simple. More complex operations or necessary tool changes lower the scoring. Table 6 summarizes the requirements for the different scorings

Table 6: Criteria scorings for the complexity of robotic motion (TAA1)

\begin{tabular}{|l|l|l|l|l|}
\hline $\mathbf{2}$ & $\mathbf{1}$ & $\mathbf{0}$ & $\mathbf{- 1}$ & $\mathbf{- 2}$ \\
\hline $\begin{array}{l}\text { Few simple standard } \\
\text { movements (only } \\
\text { translational and } \\
\text { rotational) e.g. simple } \\
\text { screws, simple grabbing }\end{array}$ & $\begin{array}{l}\text { Medium number of } \\
\text { standard movements } \\
\text { (two tool changings } \\
\text { allowed) e.g. } \\
\text { different screws }\end{array}$ & $\begin{array}{l}\text { More complex } \\
\text { movements (max 1) or } \\
\text { more tool changings } \\
\text { e.g. prying, cutting, or } \\
\text { larger grabbing }\end{array}$ & $\begin{array}{l}\text { Complex } \\
\text { movements } \\
\text { and many } \\
\text { tool } \\
\text { changings }\end{array}$ & $\begin{array}{l}\text { Very } \\
\text { complex } \\
\text { operations, } \\
\text { e.g. special } \\
\text { unplugging }\end{array}$ \\
\hline
\end{tabular}

The access (TAA2) and detection (TAA3) are two further criteria. These are strongly related to each other. For a successful automation of disassembly, it is desired that a given end effector can easily access the part or fastener. Also, a vision system must be able to detect the spot to place the disassembly tool precisely. An open view and access are preferred. Size limitations or the need for extended or angled end-effectors diminish that scoring. Shadows, a bad contrast or small part sizes lower also the detection scorings. Table 7 indicates the scorings on how a robotic end-effector could access the parts or fasteners. Table 8 summarizes the scorings on the challenges to a vision system for part detection and localization.

Table 7: Criteria scorings for the access (TAA2)

\begin{tabular}{|l|l|l|l|l|}
\hline $\mathbf{2}$ & $\mathbf{1}$ & $\mathbf{0}$ & $\mathbf{- 1}$ & $\mathbf{- 2}$ \\
\hline $\begin{array}{l}\text { Completely open, } \\
\text { any end-effector } \\
\text { could approach it }\end{array}$ & $\begin{array}{l}\text { Open, but size } \\
\text { limitations for end } \\
\text { effector, or side } \\
\text { access }\end{array}$ & $\begin{array}{l}\text { Extended end } \\
\text { effector needed (e.g. } \\
\text { extended } \\
\text { screwdriver) }\end{array}$ & $\begin{array}{l}\text { Small tool or } \\
\text { angled } \\
\text { screwdriver } \\
\text { needed }\end{array}$ & $\begin{array}{l}\text { No access at } \\
\text { all for robotic } \\
\text { end effector }\end{array}$ \\
\hline
\end{tabular}


Table 8: Criteria scorings for the detection (TAA3)

\begin{tabular}{|l|l|l|l|l|}
\hline $\mathbf{2}$ & $\mathbf{1}$ & $\mathbf{0}$ & $\mathbf{- 1}$ & $\mathbf{- 2}$ \\
\hline $\begin{array}{l}\text { Open view, no } \\
\text { shadows, good color } \\
\text { contrast and } \\
\text { relatively large part }\end{array}$ & $\begin{array}{l}\text { Open view, } \\
\text { shadows or bad } \\
\text { contrast possible } \\
\text { or medium size } \\
\text { part }\end{array}$ & $\begin{array}{l}\text { Partly hidden or } \\
\text { bad contrast and } \\
\text { shadows or smaller } \\
\text { size part }\end{array}$ & $\begin{array}{l}\text { Partly hidden } \\
\text { and bad contrast } \\
\text { or shadows } \\
\text { and/or small } \\
\text { part }\end{array}$ & $\begin{array}{l}\text { Completely } \\
\text { hidden, no } \\
\text { chance to } \\
\text { detect part }\end{array}$ \\
\hline
\end{tabular}

The automation potential for the robotic end-effector (TAA4) has been taken as another criterion for the assessment. On the one hand the rating depends on the number of studies or choices about different automation tools. The rating is influenced on the level of realization and reliability of the proposed systems. Additionally, it is taken into account how suitable such concepts are for a disassembly step. The requirements for each scoring are listed in Table 9.

Table 9: Criteria scorings for the automation potentials of the robotic end-effector (TAA4)

\begin{tabular}{|c|c|c|c|c|}
\hline 2 & 1 & $\mathbf{0}$ & -1 & -2 \\
\hline $\begin{array}{l}\text { Many choices } \\
\text { for automated } \\
\text { tool }\end{array}$ & $\begin{array}{l}\text { Some existing } \\
\text { choices for } \\
\text { automated tool }\end{array}$ & $\begin{array}{l}\text { At least one existing } \\
\text { choice for } \\
\text { automation (not } \\
\text { fully tested) }\end{array}$ & $\begin{array}{l}\text { Proposed concept } \\
\text { for automation, } \\
\text { not fully realized }\end{array}$ & $\begin{array}{l}\text { No proposed } \\
\text { concepts to automate, } \\
\text { uncertainty about } \\
\text { automation } \\
\text { possibility in future }\end{array}$ \\
\hline
\end{tabular}

The last of the TAA criteria is the material handling (TAA5). It combines the handling of the removed parts or fasteners and threads for further processing. The collection of simple fasteners into a metal bin for simple further recycling gets a scoring of two. An example would be a screw. If the parts are just metallic but small or medium size parts the rating is one. An example would be brackets. A rating of zero would be given if different materials are involved that can't be sorted such as cables with sensors, or if the parts are very large. For such large parts a crane or lifting tool could necessary. Examples are large covers or the Battery Sections. If the parts are large and there are 
different materials involved recycling is more difficult. Those parts get a rating of minus one. If parts are very large, have an unwieldy shape or if hazardous materials are involved then the rating would be minus two. An example would be a cooling plate that contains an easy flammable coolant such as R1234yf.

\subsubsection{Assessment for Hybrid Vehicle Battery}

The ten criteria have been applied on the 19 steps for disassembly of the Audi Q5 HEV battery (see section 2.2.2). For each criterion the calculations and assumptions have been documented. Table 10 shows the assessment on the first step of the Audi Q5 hybrid vehicle battery disassembly.

Table 10: Assessment on the Unscrewing of covers for the Audi Q5 hybrid vehicle battery

\begin{tabular}{|c|c|c|}
\hline Criteria & Comments & Scorings \\
\hline NA 1 & $\begin{array}{l}\text { Move, position, unscrew, move, grasp, bring, release for every bolt/nut }+ \text { tool } \\
\text { change approx. } 7 * 20=140 \text { movements }\end{array}$ & 2 \\
\hline NA 2 & $\begin{array}{l}5 \text { seconds for every bolt/nut, approx. 120s in total with screwdriver grabbing/ } \\
\text { tool change }\end{array}$ & 2 \\
\hline NA 3 & No high voltage or chemical dangers at that point, only sharp edges possible & -1 \\
\hline NA 4 & Very low weights, just screws/bolts and nuts, some bending to reach screws & -1 \\
\hline NA 5 & $\begin{array}{l}\text { Necessary to reach cells because no other possibilities to come to } \\
\text { cells/modules }\end{array}$ & 2 \\
\hline TAA 1 & $\begin{array}{l}\text { Tool changing seems necessary, but only translational und rotational. Simple } \\
\text { standard movements }\end{array}$ & 1 \\
\hline TAA 2 & Access form sides and bottom needed, more difficult, but open & -1 \\
\hline TAA 3 & Also, detection on sides and bottom needed & 0 \\
\hline TAA 4 & $\begin{array}{l}\text { Some choices e.g. R. Li et al., "Unfastening of Hexagonal Headed Screws by a } \\
\text { Collaborative Robot," IEEE Trans. Autom. Sci. Eng., pp. 1-14, } 2020 \text { [26] }\end{array}$ & 1 \\
\hline TAA 5 & Just collection of nuts/bolts & 2 \\
\hline
\end{tabular}


Approximately 20 bolts or nuts have to get loosened and collected. Due to the high number of fasteners the scoring on the movements and time criteria is high. There is no danger of chemical hazards or electrical shocks on that first step. So, the scoring on danger is low, just sharp edges could pe present. The weights are also low, only some bending seems necessary, while the step has a high importance in order to reach the most valuable parts. The NA scoring adds up to 40 . That recommends the necessity of automation for that step.

The required movements are standard unscrewing movements, but a tool change is necessary. The scoring on the access is low, because the fasteners are also located on the side and bottom. That also complicates the detection. The scoring automation potential is higher, because there have been some approaches on locating and unfastening bolts or nuts[6], [27], [52], [53]. Finally, the material handling is easy. Fasteners are light parts that are collected in a bin for further recycling. A scoring on 30 for TAA suggests the possibility that there is a high chance to realize the automation, even if access and detection below the battery are more difficult to achieve.

Figure 34 shows the result for 18 of the disassembly steps. For most of the steps it seems necessary and technically possible to automate those. Some extreme examples need further explanation. For example, Step 16, (Unscrewing of nuts on the cell contacts) has a TAA of 90. That high scoring refers to a relatively simple unscrewing operation where the fasteners are accessible from the top. There is a good contrast in color and shape of the fasteners for detection, while there are choices for automation of unscrewing operations. As discussed before the material handling of fasteners is simple. 
Another example is Step 5, (disassembly of the plug connection between the cell controllers and the BMS) that scores a -10 in both. It is neither necessary nor possible to automate this step. In the framework of a human-robot workstation [6], this would be a typical task for the human worker. The low NA scoring results from the low weights and few amounts of movements. The low TA scoring is caused by a difficult access and detection and uncertainty about automation potentials for the robotic end-effector.

Steps 2 and 8 score negative on NA because those are fast cover removal operations. But such relatively simple grabbing operations score relatively high on TAA because there are several automation approaches. If a gripper is installed it could be adjusted for such steps to save some extra worker's time, even if it is not as necessary as for other steps.

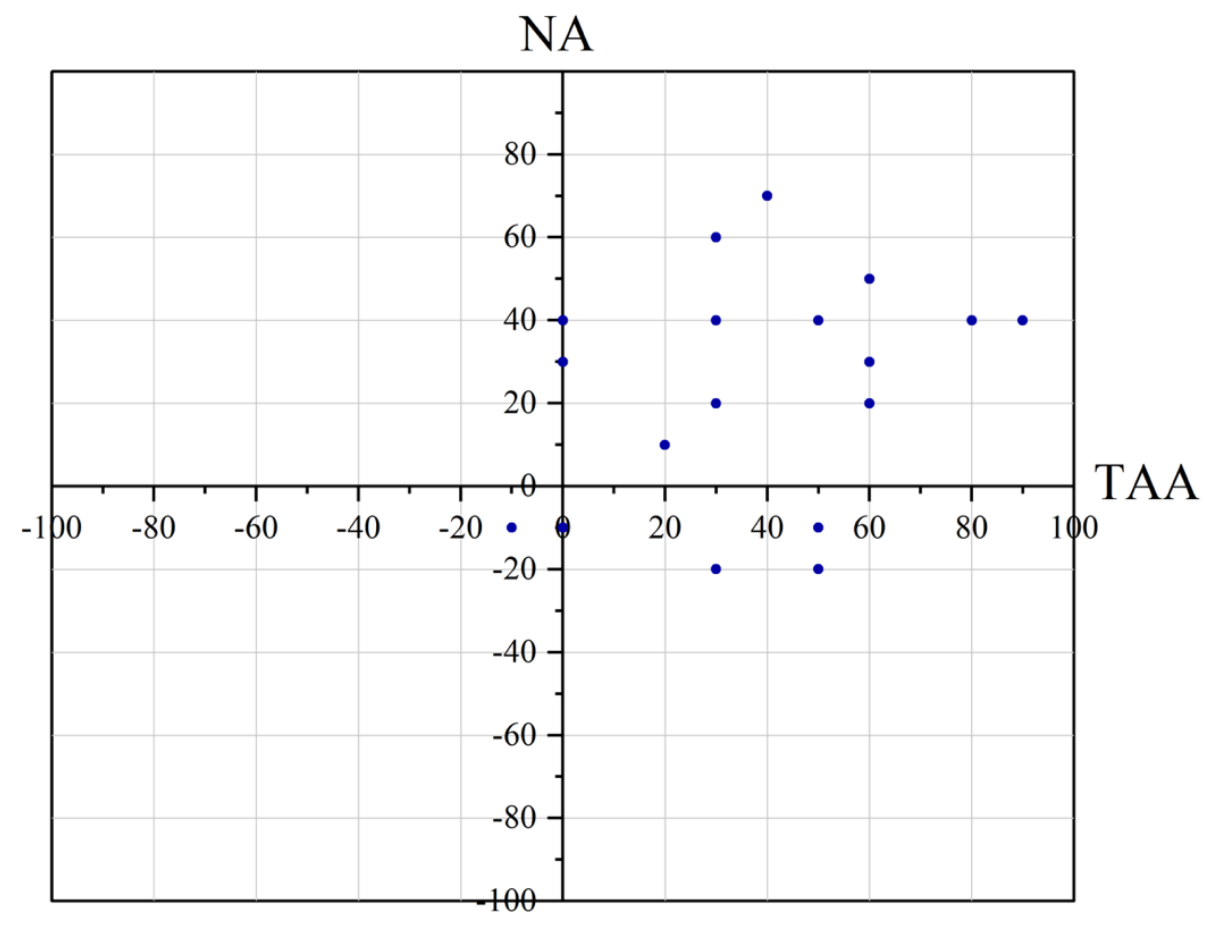

Figure 34: Assessments of disassembly steps for Audi Q5 hybrid vehicle battery 


\subsubsection{Assessment for Battery Electric Vehicle Battery}

Similar to the assessment of the steps for the hybrid electric vehicle the assessment has also been applied on the 46 steps for the disassembly of the EV battery (see Figure 35 and a table with all results in Appendix 6.1.1). From a first view the picture looks relatively similar to the hybrid vehicle battery. A relatively extreme example is D39 (described in detail in 3.1.3). This step has an NA scoring of 90 because of the extreme necessity due to the importance of separating the Battery Sections and the high weight of those. However, the TAA scoring is lower but still positive. It is more difficult to automate a complex lifting operation for heavy parts.

D1 is an example for a step with a very high automation potential. The TAA scoring is 90 . As discussed before there are several approaches to automate unscrewing operations with open view and access. The NA scoring of 40 is lower because in that first step there are no hazards due to high-voltage or chemicals as there could be in later steps.

The two steps discussed before both scored positive on NA and TAA scales, so those could be and should be automated. Contrary to that, D10 scores -40 on TAA and 30 on NA scales. On the one hand that step should be automated because it is necessary to reach the valuable battery cells and also the relay center which could be reused. But on the other hand, the operation is very difficult to automate. There is not much space for a robotic end-effector and the pats are difficult to detect. Also, different grabbing tools seem necessary. Probably it would be easier to perform this step by a human worker.

D42 is an example for a step that could be automated but is not necessary to automate. The grabbing of the braces scored -30 in NA in 70 in TAA. The high TAA is 
based on the relatively simple grabbing operation with just a little bit larger part but with open access. The low NA is based on the fact that the Battery Sections are already taken out, so there are no HV or chemical hazards anymore. Furthermore, this step is not necessary in order to get access to the battery modules. Those are already taken out and no more expensive materials can be disassembled with this step. It depends on the requirements for further processing, if the materials remaining in the battery tray should be separated. For the braces that could be done relatively easy by a robot.

The disassembly of the nuts for the coolant hoses are described with D12. That step is not necessary in order to gain access to the battery cells and it could be done relatively quick by a human worker. That leads to an NA score of -20. From a technical point of view, it is difficult to automate this step due to the need for special tools and the lack of previous studies on such automation. The TAA scoring is - 30 . This step should still be performed by a human worker or left out completely.

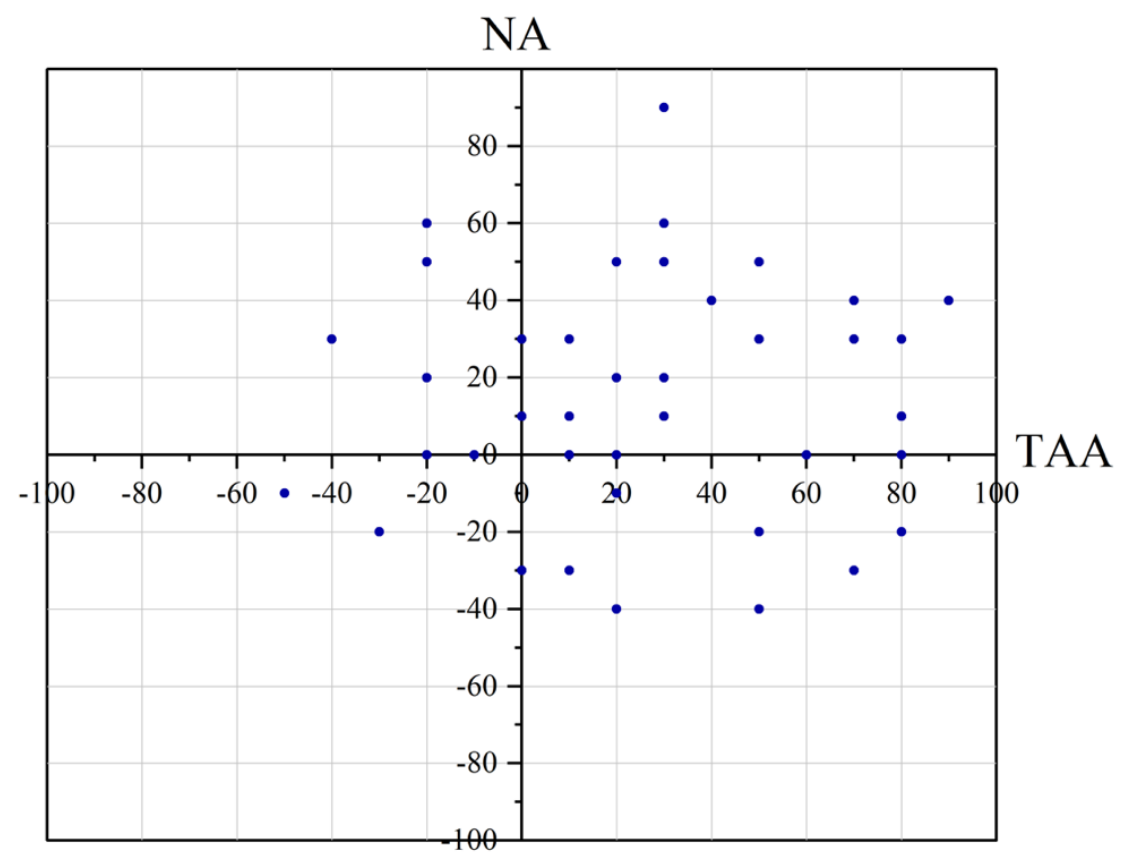

Figure 35: Assessment of disassembly steps for 2017 Chevrolet Bolt battery 


\subsubsection{Comparison of the Assessment of the different Battery types}

In this section the results from the analysis on the HEV and the BEV battery will be compared with the results from Herrman et al. [60] who performed analysis on several different kinds of EV batteries. Figure 36 shows the results of that study.

First comparing the analysis on the BEV (see Figure 35) the HEV battery (see Figure 34), one can notice the higher number of necessary disassembly steps for the BEV battery. This results from the larger number of parts and higher complexity of the BEV battery. Another factor is that the Chevrolet Bolt BEV battery has a more complex design; the fifth Battery Section is placed above the fourth one. This requires a second cooling plate below the fifth Battery Section and several more parts.

Methodologically there is also an important difference. Wegener et al. [6] analyzed the disassembly down to the level of modules and then continued the disassembly of the modules down the level of battery cells. The disassembly steps of the Chevrolet Bolt battery describe the disassembly just down to the level of Battery Sections ( 1 Section $=2$ Modules). The subsequent steps describe the further disassembly of the remaining part in the battery tray. Because of that the last steps of the BEV battery score lower on NA. Those steps are not needed to reach the battery cells. However, for the hybrid vehicle battery all proposed steps are necessary to reach the cells.

Another difference is the size. BEV batteries are much larger than HEV batteries. As discussed before the larger size and higher number of parts in the BEV battery leads to a larger number of disassembly steps. But the larger size leads also to a higher number of motions for each step. For example, there are 56 bolts around the top cover for the BEV 
battery, but just 20 fasteners for the covers surrounding the Audi Q5 hybrid battery. This leads to comparable higher NA scoring for the EV battery due to a higher number of motions and a longer manual disassembly time (NA1 and NA2).

The results of the investigations by Herrman et al. [60] for many different BEV, PHEV and HEV batteries (see Figure 36) do not score a NA above 50 for any disassembly step. Those investigations were executed with a more detailed criteria catalogue. The results of our investigation (see Figure 35) score a NA of 50 or higher for several disassembly steps. From that it could be concluded that the simplified criteria catalogue produces more extreme scorings. There are more disassembly steps with TAA or NA above 50 or with negative values for the BEV assessment with the simplified catalogue. The higher NA scorings could be a caused by the focus on disassembly time and dangers. There are many repetitive disassembly steps in the BEV disassembly that score high on NA1 and NA2 because of the disassembly time and number of motions and high. High scorings on NA3 result from the HV dangers for all nearly all steps in between the "Top Cover" removal and the extraction of the battery modules. NA5 receives high scorings for many steps because those are necessary for reaching the most valuable materials, the battery modules and cells. Because of the high scorings in those four categories there are high NA scorings for many disassembly steps. Furthermore, the TAA scorings are lower in the study with the detailed criteria catalogue. It is also suggested to only automated the three steps with a TAA above 50 and positive NA. These operations are the handling of the battery, the extraction of the cells and the extraction of the modules [60]. With our simplified criteria catalogue those operations are rated worse in TAA because lifting operations are categorized as more complex. But in comparison to that previous study 
[60] our assessment strongly recommends automating unscrewing operations. Most of those have a very high TAA and high NA. Technical progress in automation of unscrewing and other operations is another reason for different TAA scorings. The investigations by Wegener et al. [6] also suggest the automation of unscrewing operations but that study did not provide ratings for single disassembly steps.

Our results (see Figure 35) show that the ratings of the disassembly steps are placed into all four quadrants of TAA and NA combinations. That underlines the need for human-robot collaboration because some disassembly steps are very difficult to automate, so the human has to perform these disassembly steps or at least teach the robot. Those disassembly steps that score high in TAA and NA should definitely be automated, and an automation could be realized. Disassembly steps with a high TAA rating but low NA rating could easily be automated but it is not necessary to automate those. An example for such a step is D42, the unscrewing of the screws for the Braces. It should be decided case by case, if such a step should be automated. Economic considerations are most important for such decisions. In the case of D42, an automated screwdriver is already included in the robotic end-effector for several more important disassembly steps. Bearing this in mind, D42 should be automated, because it does not require a lot of effort to do so. For disassembly steps that score low on both TAA and NA a human worker is the better choice for performing those because it will be difficult but not necessary to automate. Disassembly steps that score high on NA but low on TAA, it is recommended to automate, but I could be difficult to do it. An example is D10, a very complicated grabbing operation. For such a disassembly step a human worker is still the better choice. Investigations on technical realization of such steps are necessary. 
Concluding, there is an even higher need for automations in EV battery disassembly in comparison to HEV/PHEV batteries due to the larger size, higher weight and the resulting expected higher disassembly time for manual disassembly. The resulting higher repetition in many steps strongly recommends automation for those. From a technical point of view some disassembly operations are more difficult because of the weight, size and complexity. Those include the handling of the battery and the handling of the battery modules. Strong efforts on reliable automated systems for such steps are required because those operations with heavy weights are also not suited for human workers. There are still some non-repetitive or difficult disassembly operations in BEV battery disassembly were humans are superior over robots. With the large variety of differently assessed disassembly steps, a human-robot workstation (see [6]) is the suiting concept for the disassembly BEV and HEV/PHEV batteries. The similarities of BEV and HEV/PHEV disassembly suggest to disassembly all types in the same factory. But there should be an own larger sized disassembly station for the disassembly of the BEV batteries. The extracted battery modules of all battery types could be disassembled at the same disassembly station. A further discussion on the layout will be shown in the following chapter. 


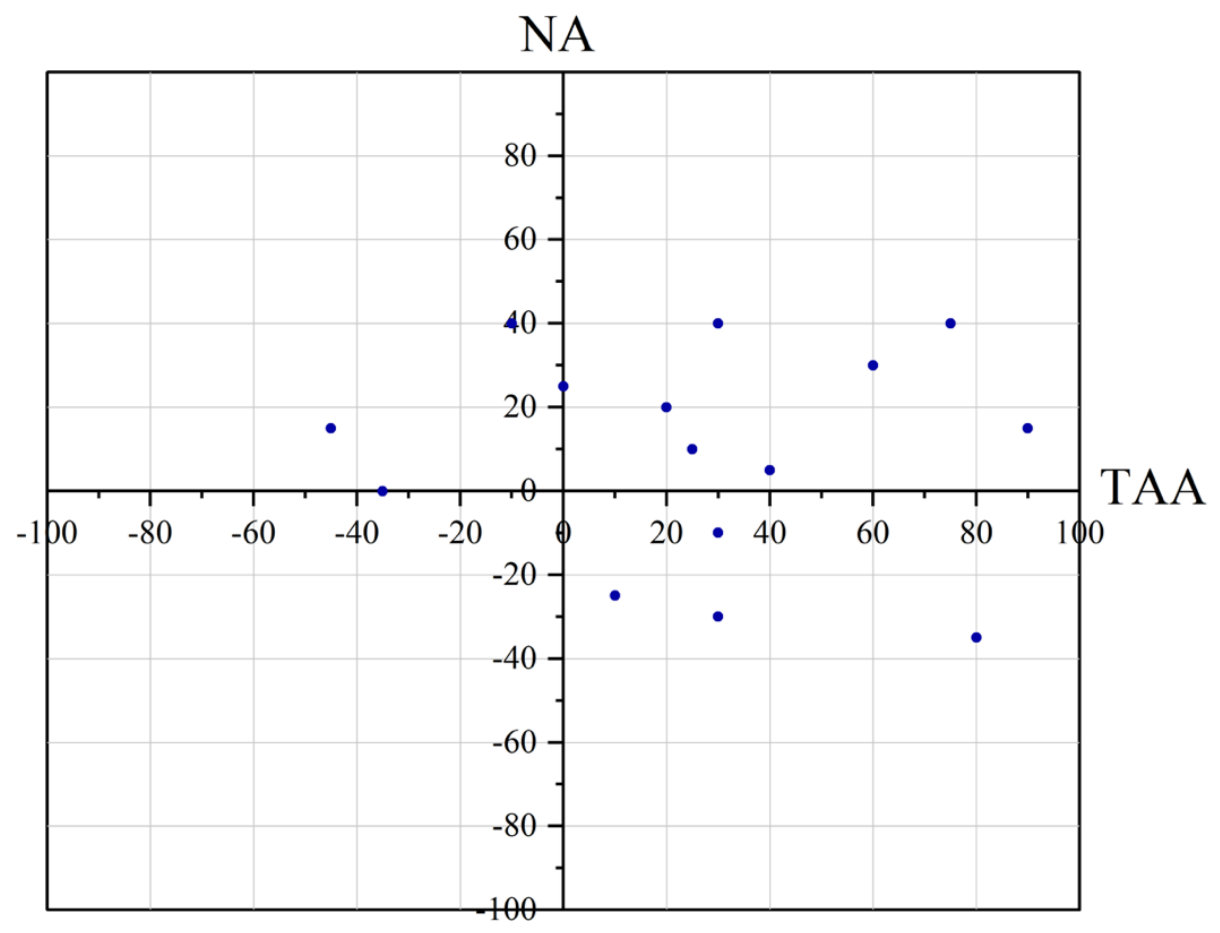

Figure 36: Assessment of disassembly steps for several BEV and HEV/PHEV batteries, adapted from [60] 


\section{Disassembly Work-Cell Concepts}

This Chapter will present a comparison of disassembly work cell concepts. It uses the example of the 2017 Chevrolet Bolt battery. Two different concepts for disassembly of a simplified EV battery were modeled with a simulation software. The results of the simulation will be used for the comparison and some further layout ideas will be presented.

\subsection{The Visual Components software}

The employed simulation software, Visual Components is mainly used for factory planning. 3D shapes can be created and a factory with robots, machines and conveyors can be modeled. The software has a large variety of robots by different manufactures that can be modeled. The Works library was used, and the library gives the possibility to create pre-designed shapes in Works Process fields at certain 3D positions and assigning transport tasks to the robots. Each Works Process field can "feed" parts so the robot takes away parts there, or "need" parts, so the parts get transported there. Also, parts can be created in patterns, so starting from one position a defined number of those objects appears with the same distance each in chosen directions. That function is useful for creating repeated objects such as bolts.

\subsection{Model of the EV Battery}

For the simulation we decided to create a simplified model of the 2017 Chevrolet Bolt battery. Only some parts and fasteners for demonstrating the most important operations and the main problems, for example the large size were modeled. Table 11 lists the modeled parts and fasteners. All parts are modeled with simple geometric shapes 
Table 11: Modeled Parts and Fasteners for Disassembly Simulation

\begin{tabular}{|c|c|c|c|}
\hline Part or Fastener & Quantity & $\begin{array}{l}\text { Part in real } \\
\text { EV battery }\end{array}$ & Picture of the model \\
\hline Battery Tray & 1 & P81 & \\
\hline Top Cover & 1 & $\mathrm{P} 1$ & \\
\hline Bolts for Top Cover & 22 & F1-56 & \\
\hline $\begin{array}{l}\text { Bolts for Electrical } \\
\text { Connector Housing }\end{array}$ & 4 & F357-360 & \\
\hline $\begin{array}{l}\text { Electrical Connector } \\
\text { Housing }\end{array}$ & 1 & P62 & \\
\hline Cable & 1 & e.g. P16 & \\
\hline Bolts for Brackets & 4 & e.g. 248 & \\
\hline Brackets & 4 & e.g. P32 & \\
\hline $\begin{array}{l}\text { Battery Modules or } \\
\text { Sections }\end{array}$ & 3 & e.g. P36 & \\
\hline
\end{tabular}

with sizes corresponding as best as possible to the actual parts in the real 2017 Chevrolet Bolt EV battery. The Battery Tray for example is modeled as a hollow shape with a wall thickness of $20 \mathrm{~mm}$, a height of $150 \mathrm{~mm}$, a length of $1600 \mathrm{~mm}$ and a width of $1000 \mathrm{~mm}$. 
Two different types of fastener sizes are used. Instead of the bolts in the real EV battery simple nuts are modeled. The bolts for the Electrical Connector Housing in the front are slightly larger. That difference is taken for simulating the use of different screwdrivers. Originally there are 56 bolts that fix the Top Cover with 6 of them in on the higher part of the Top Cover. We just modeled eleven bolts on each side and 4 on the higher part. So, there is a total of 26 bolts. The Electrical Connector Housing was modeled as a simple rectangle on the front side of the Battery Tray. 4 Bolts are placed for fixing it. Those help to simulate the more difficult loosening of fasteners from the side. Inside the Battery Tray, below the Top Cover we placed three simple Battery Sections. On the first of those, four Brackets with one Bolt each are placed. The Brackets with their bolts have to be removed first before the Battery Sections can be removed. Furthermore, a Cable is placed inside the Battery Tray. As discussed before, there are some cables inside EV batteries and the disassembly of clips that fix the cables or the cutting of the cables are challenging tasks for automation. Therefore, this task is assigned to the human worker in the simulation. A human task also helps for simply simulating aspects of human-robot-collaboration. Figure 37 shows the model of the BEV battery.
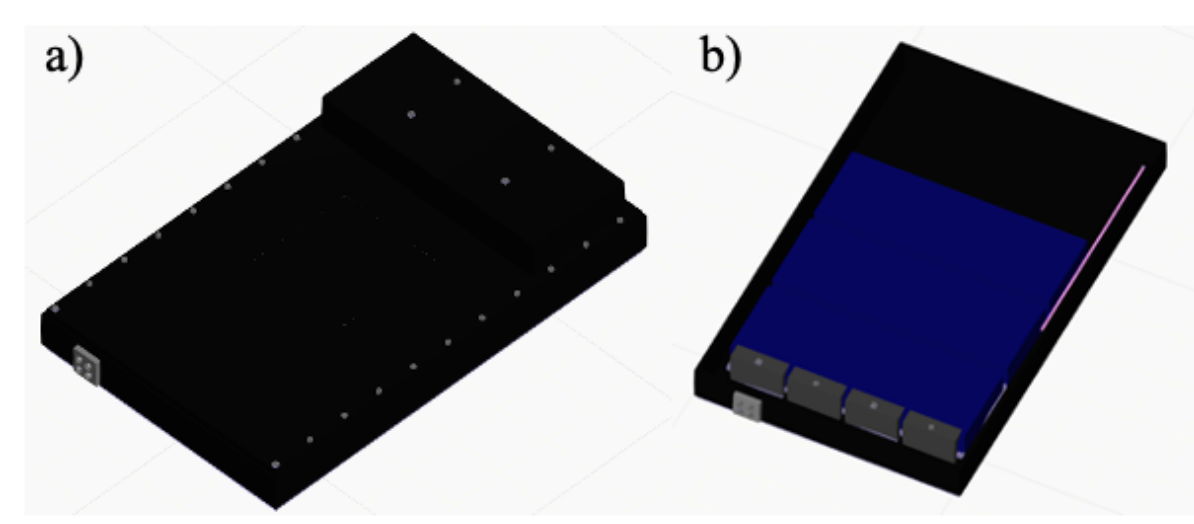

Figure 37: Modeled BEV Battery, a) Complete battery, b) Battery with removed Top Cover 


\subsection{Modeled Tools}

The previously described disassembly of EV batteries needs different tools. There is the need for screwdrivers and nutrunners with different bit sizes. Cutting tools are necessary for covers and hoses and prying tools are required for the opening of covers and clips. The large variety of part sizes and shapes creates the need for different flexible grabbing tools. The simple simulation will use a long and thin suction gripper instead of a screwdriver. There are two different sizes of bolts, so a tool change can be simulated. The time for aligning with the fastener and loosening is simulated by a delay of two seconds in the picking. For the grabbing task, two different sizes of suction grippers are used. A large suction gripper grabs the Top Cover and the Battery Sections, while a smaller suction gripper is responsible for the Brackets and Electrical Connector Housing as smaller parts. For the grabbing of the large parts a pick delay of 5 seconds represents the more complex adjusting of a real grabbing tool for large parts. A tool changing station is modeled by a simple table on which the required tools are placed. The modeled robots exchange the tools automatically by placing the old one at its default position and picking up a new tool. The human worker can pick and place parts. In these simulations no vision system is provided. The positions of the parts and fasteners are known by the robot.

\subsection{Layout with one large Cartesian Gantry Robot}

One of the two proposed modeled layouts use a large gantry robot. The Generic Cartesian Robot by Visual Components is used. The frame of the gantry robot (see Figure 38) traverses the complete BEV battery, the tool changing station and placement areas for two conveyors. The cartesian gantry robot can move its end effector in $\mathrm{x}-, \mathrm{y}-\mathrm{and} \mathrm{z}-$ 
directions. The end effector can reach parts and fasteners from the top and the sides. The robot is able to perform all disassembly tasks that are assigned to it. The technical possibilities for task automation are discussed in Chapter 3. The conveyor on the left side collects all metallic fasteners such as screws, nuts and bolts. The larger right conveyor is used for the collection of the larger and medium size parts such as the Top Cover, the Battery Sections or the Brackets. Those parts can be sorted in a later process step for recycling. The human can reach all parts of the battery. The gantry robot is not suited for human-robot- collaboration. Therefore, the robot must wait in a certain default position until the human has performed its task and left the disassembly area. That can be indicated with the help of a vision system or by a signal of the human worker. In this layout, the only task for the human is the removal of the cable. After removing the cable the human places it in an area for collection of electronic waste.

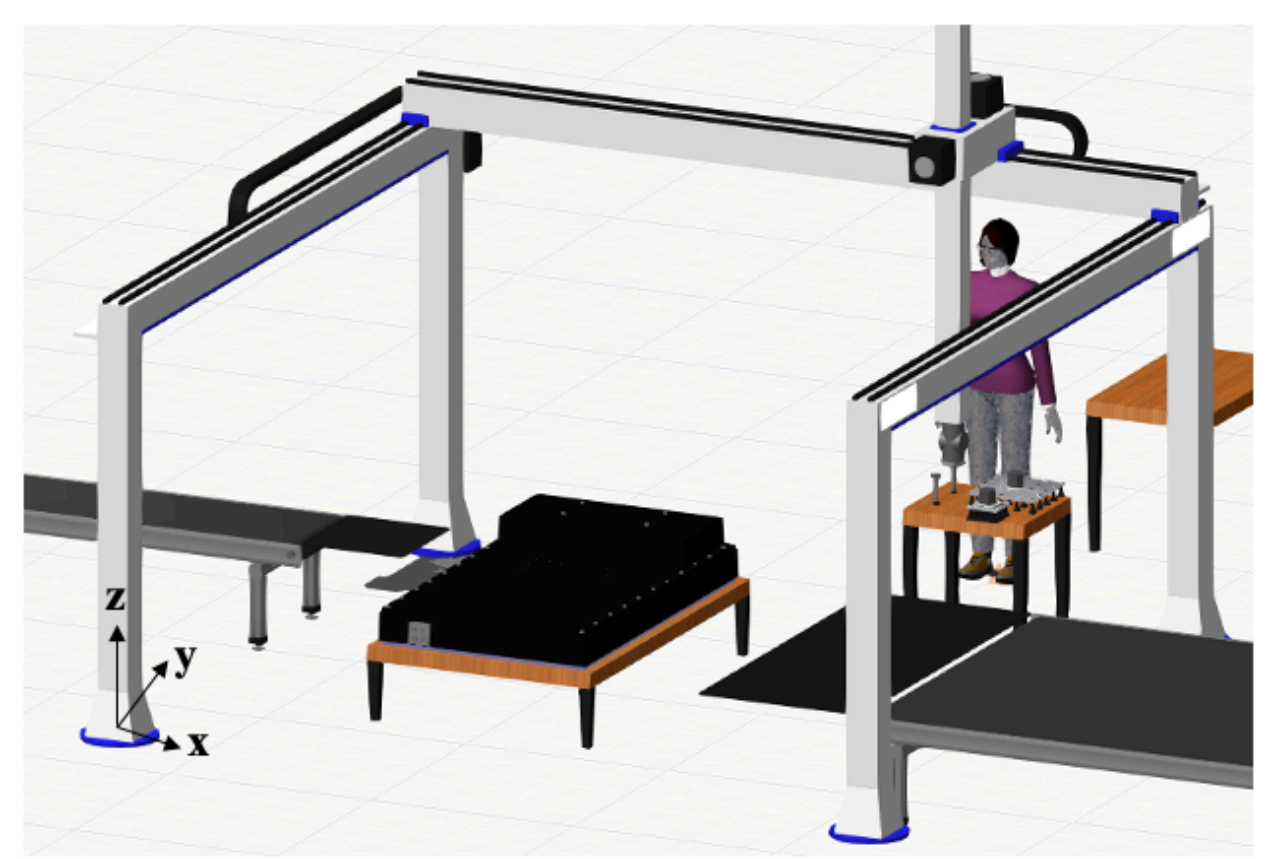

Figure 38: Layout with one large cartesian gantry robot 


\subsection{Layout with two Collaborative Robots}

In this layout (see Figure 39) two collaborative robots are on placed linear slides on each side of the BEV battery. The model of the collaborative UR10e by Universal Robots is taken for this layout. Each robot got its own tool changing station (Figure 40a) and its own conveyor for transporting out the disassembled parts and fasteners. Both robots can work at the same time. The robots disassemble the parts and fasteners on their side such as the Bolts around the top cover (Figure 40c). With the help of the linear slides (Figure 40d), the $U R 10 e$ robots can reach all parts on their sides of the battery. The example battery has an approximate length of $1600 \mathrm{~mm}$ and a width of $1000 \mathrm{~mm}$ while the robots have a range of $1300 \mathrm{~mm}$ [98]. So, the robots can reach the middle of the battery in $\mathrm{x}-$ direction and can be positioned over the complete length of the battery in y-direction. The robot on the left side also unfastens the bolts for the Electrical Connector Housing and grabs this housing. After that again both robots work in parallel and unscrew the bolts for the brackets (Figure 40b). The linear slides are used to place the robots close to certain parts and fasteners because of the small workspace range of UR10e. The robots can work in parallel with the human if their motions paths do not interfere. The small UR10e robots have a low payload, so they cannot handle heavy parts such as the Top Cover or the Battery Sections. So, in addition to the cable the human worker also disassembles the Top Cover (Figure 40e). The Battery Sections cannot be disassembled with the presented layout because of the low payload of $10 \mathrm{~kg}$ [98] for UR10e robots Therefore, an additional crane would be necessary. For example the crane used by Kelly [21] could be used for manual lifting of the Battery Sections. While the human disassembles the Top Cover and the cable, the right robot has to wait until it can unscrew the bolts for these Brackets. The 
left robot can disassemble the Electrical Connector Housing in parallel. Disassembly time is saved due to parallel working.

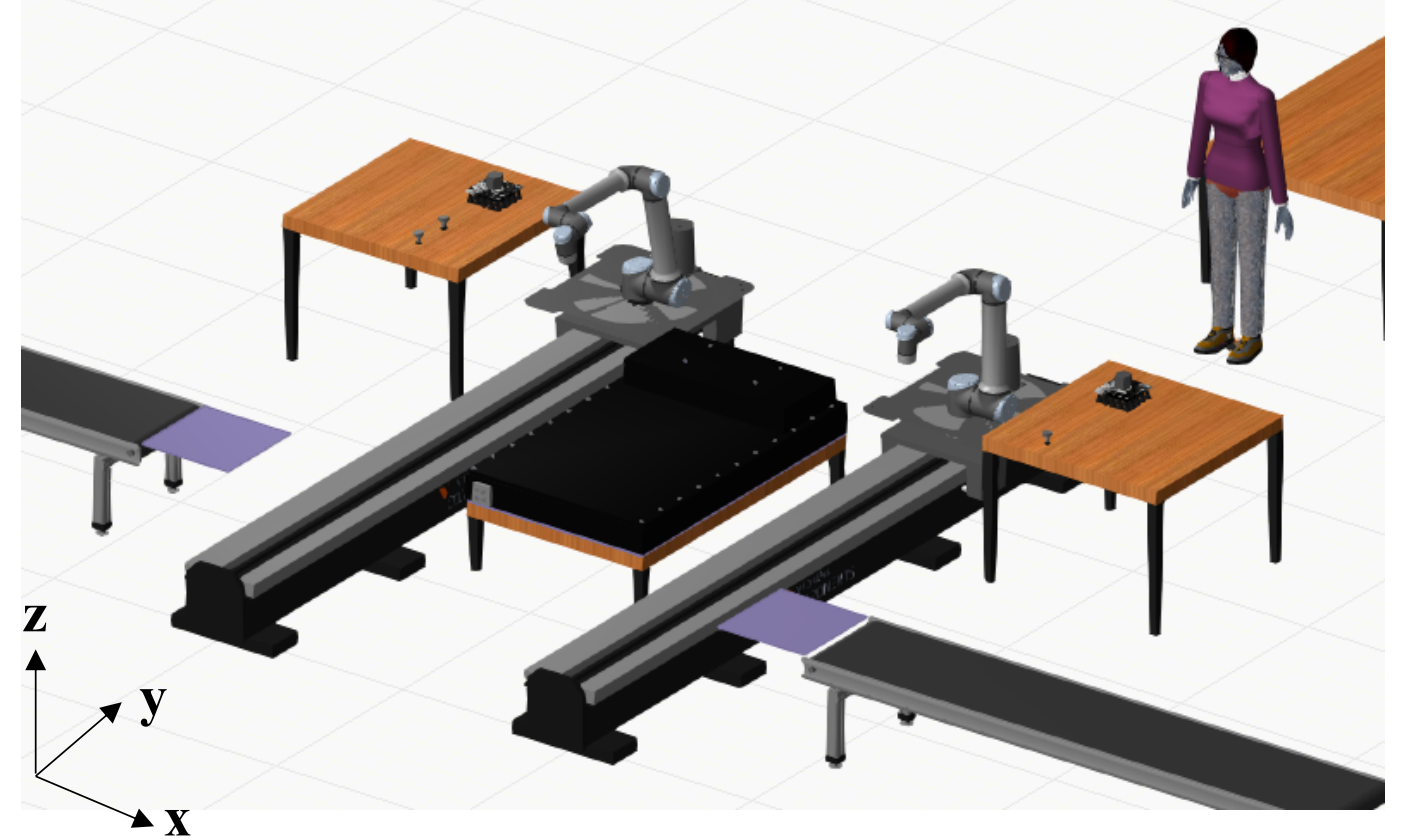

Figure 39: Layout with two collaborative robots
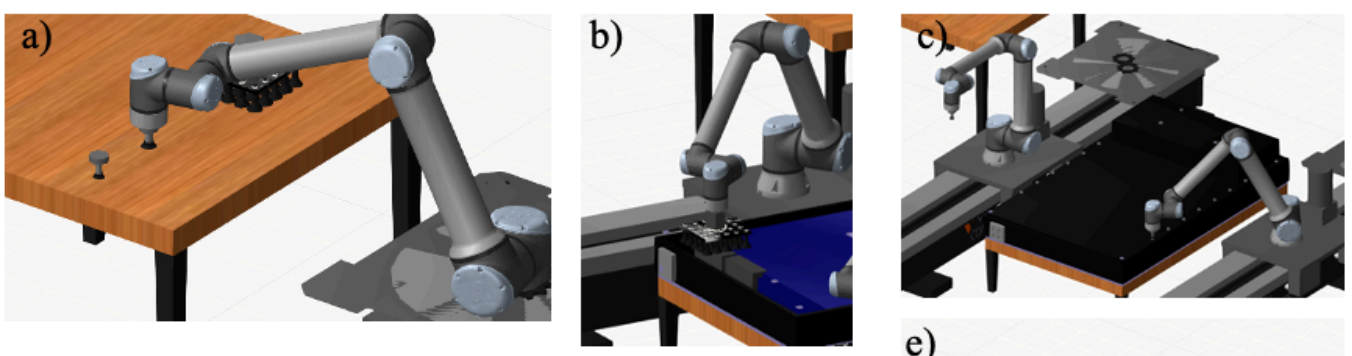

e)

d)
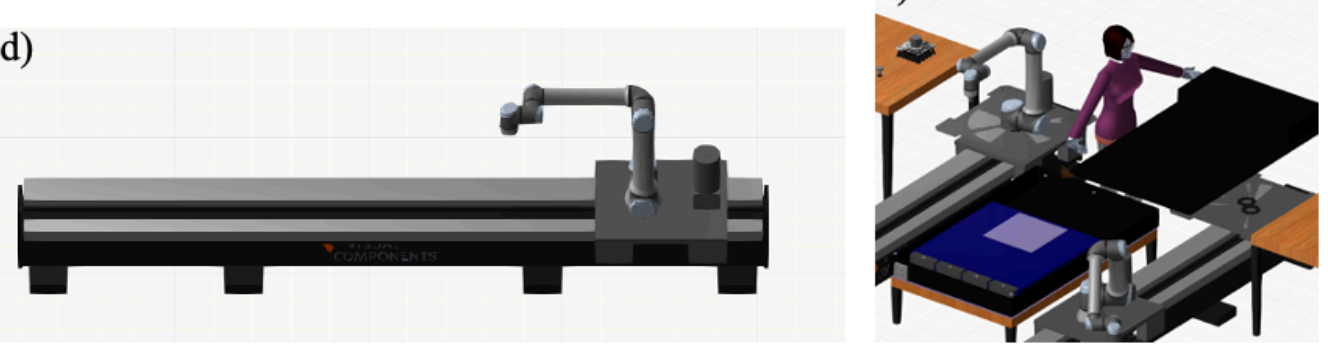

Figure 40: Collaborative robots layout: a) Changing of Unscrewing Tool, b) Unscrewing of Bolts for Brackets, c) Unscrewing of Bolts around Top Cover, d) Linear slide with a collaborative UR10e robot on it, e) Disassembly of the Top Cover by the human worker 


\subsection{Disassembly Sequence for the modeled BEV Battery and Comparison of the two Disassembly Cell Layouts}

The two proposed layouts will be compared in terms of disassembly time and ability to reach all parts and fasteners and also the possibility for human-robot collaboration. First, the disassembly sequence will be described.

\subsubsection{Proposed Disassembly Sequence}

For the disassembly of the modeled BEV battery the disassembly sequence (see Table 12) was simply determined by constraints of the parts arrangement and was optimized manually (some disassembly steps are shown in Figure 41). The disassembly starts with the removal of the Bolts for the Top Cover. The next task is the removal of the bolts for the Electrical Connector Housing. Therefore, the screwdriver has to be changed, because these bolts are larger. In the layout with the two collaborative robots this disassembly step is performed simultaneously with the two next ones. These are the removal of the Top Cover and the removal of the Cable. The Cable removal is done manually in both layouts. After the removal of the Top Cover, the Bolts for the Brackets are reachable. Following the removal of these Bolts, the Brackets and Electrical Connector can be removed. For these two parts the same suction gripper is applied. The last task is the removal of the three EV Battery Sections. This task cannot be performed by the UR10e robots or the human worker because of the high weight of the Battery Sections of more than $50 \mathrm{~kg}[21]$. 

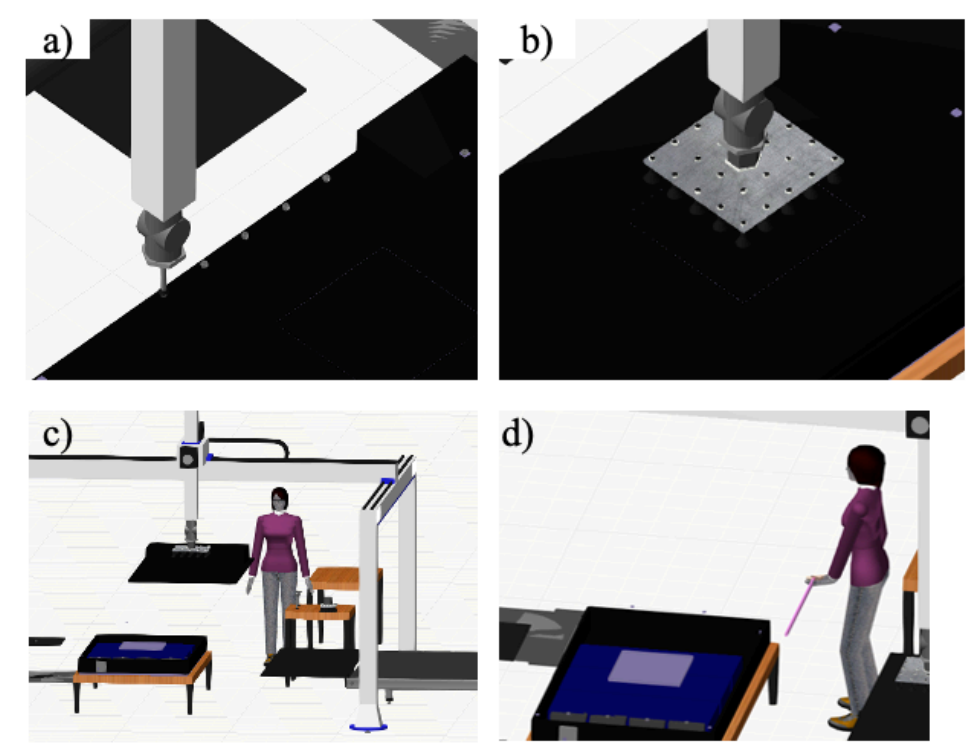

Figure 41: Examples of disassembly steps: a) Unscrewing of Bolts around the Top Cover, b) Grabbing of the Top Cover by the Suction Gripper, c) Delivering the Top Cover to the Conveyor, d) The human worker disassembling the Cable

Table 12: Disassembly Sequences for modeled layouts

\begin{tabular}{|l|l|l|l|l|}
\hline \multicolumn{2}{|l|}{} & \multicolumn{2}{|l|}{ Gantry Robot } & \multicolumn{2}{l|}{ Collaborative UR10e Robots } \\
\hline $\begin{array}{l}\text { Disassembly } \\
\text { step }\end{array}$ & $\begin{array}{l}\text { Disassembled } \\
\text { Fasteners or Parts }\end{array}$ & Automated & $\begin{array}{l}\text { Disassembled } \\
\text { Fasteners or Parts }\end{array}$ & Automated \\
\hline 1 & $\begin{array}{l}\text { Bolts around the Top } \\
\text { Cover: 26 Bolts }\end{array}$ & Yes & $\begin{array}{l}\text { Bolts around the Top } \\
\text { Cover: 26 Bolts }\end{array}$ & Yes \\
\hline 2 & $\begin{array}{l}\text { Bolts for Electrical } \\
\text { Connector Housing: } \\
4 \text { Bolts }\end{array}$ & Yes & $\begin{array}{l}\text { Bolts for Electrical } \\
\text { Connector Housing: } \\
4 \text { Bolts }\end{array}$ & Yes \\
\hline 3 & Top Cover & Yes & Top Cover & No \\
\hline 4 & Cable & No & Cable & No \\
\hline 5 & $\begin{array}{l}\text { Bolts for Brackets: } \\
4 \text { Bolts }\end{array}$ & Yes & $\begin{array}{l}\text { Bolts for Brackets: } \\
4 \text { Bolts }\end{array}$ & Yes \\
\hline 6 & $\begin{array}{l}\text { Brackets for Battery } \\
\text { Section: 4 Brackets }\end{array}$ & Yes & $\begin{array}{l}\text { Brackets for Battery } \\
\text { Section: 4 Brackets }\end{array}$ & Yes \\
\hline 7 & $\begin{array}{l}\text { Electrical Connector } \\
\text { Housing }\end{array}$ & Yes & $\begin{array}{l}\text { Electrical Connector } \\
\text { Housing }\end{array}$ & Yes \\
\hline 3 Battery Sections & Yes & 3 Battery Sections & $\begin{array}{l}\text { Task not } \\
\text { performed }\end{array}$ \\
\hline
\end{tabular}




\subsubsection{Comparison by Disassembly Time}

The simulation provided the process flow of the disassembly of the BEV battery. From that the process times for each disassembly step were listed. Figure 42 shows the disassembly time of the layout with one large gantry robot. The first disassembly step, the unscrewing of 26 Bolts, consumes more than half of the total disassembly time due to the high number of operations. The fourth step, the cable removal is the only disassembly step that is not automated, and it takes ten seconds. All together the disassembly of this simple model is finished after 258 seconds of disassembly.

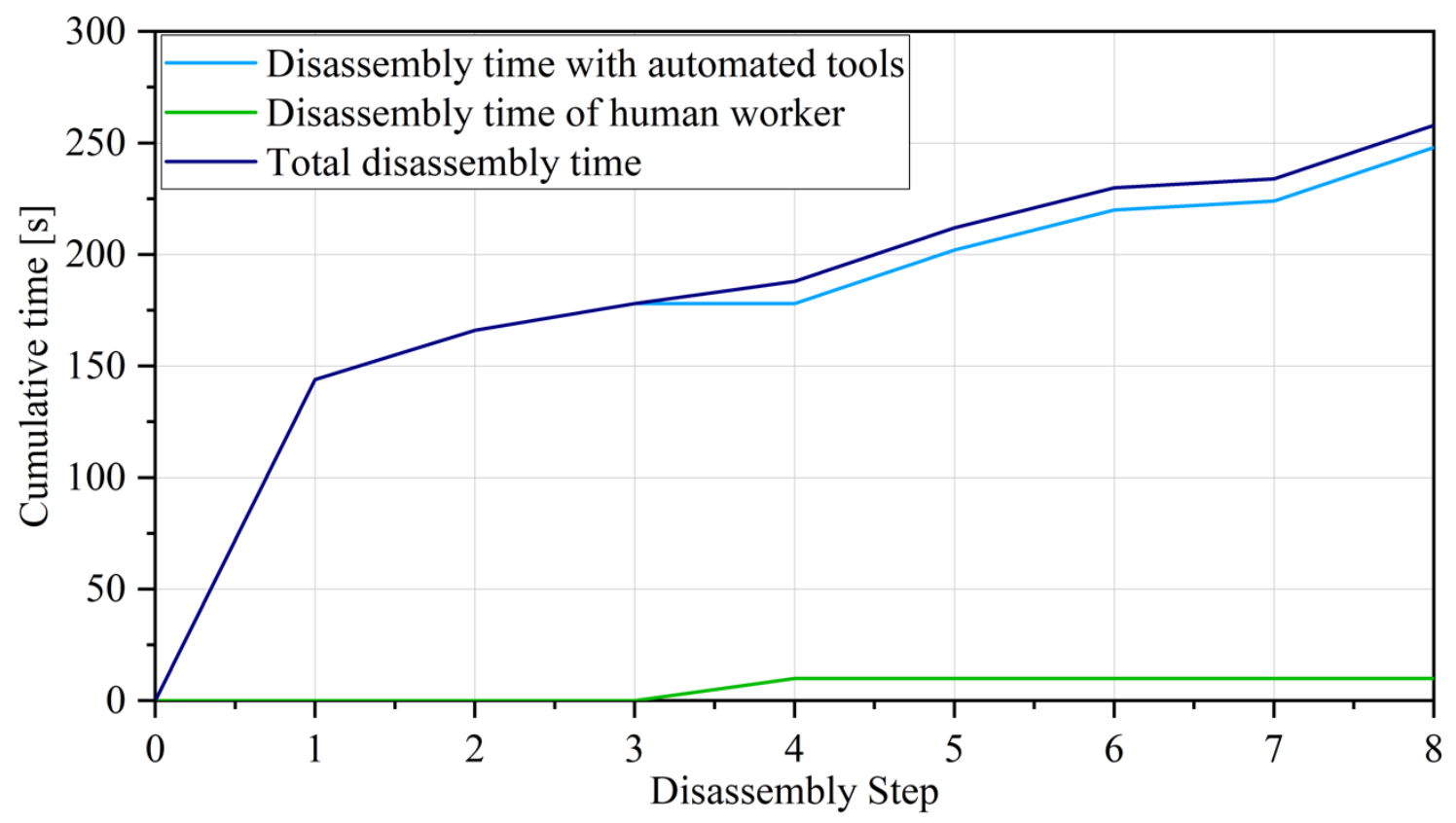

Figure 42: Disassembly time for disassembly with Gantry robot for modeled Battery

Figure 43 presents the disassembly time for the layout with two collaborative UR10e robots. The first, fifth and sixth steps are done by both robots operating in parallel, so, for example, each robot only disassembles 13 Bolts for the first disassembly step. It is indicated, that the third and fourth step do not consume time. The reason is the humanrobot-collaboration. The UR10e robots can work parallel to the human in the same area. 
So, the left robot performs the unscrewing of the four Bolts for the Electrical Connector Housing (step 2) while the human worker removes the Top Cover (step 3) and the Cable (step 4). These two tasks take less time than the four bolts. In total the disassembly consumes 254 seconds but the eighth step, the removal of the three Battery Sections cannot be performed. The gantry robot only needs 234 seconds for the first seven disassembly steps. So, even if two collaborative robots work in parallel and the robots can work in parallel to the human, the gantry robot is slightly faster.

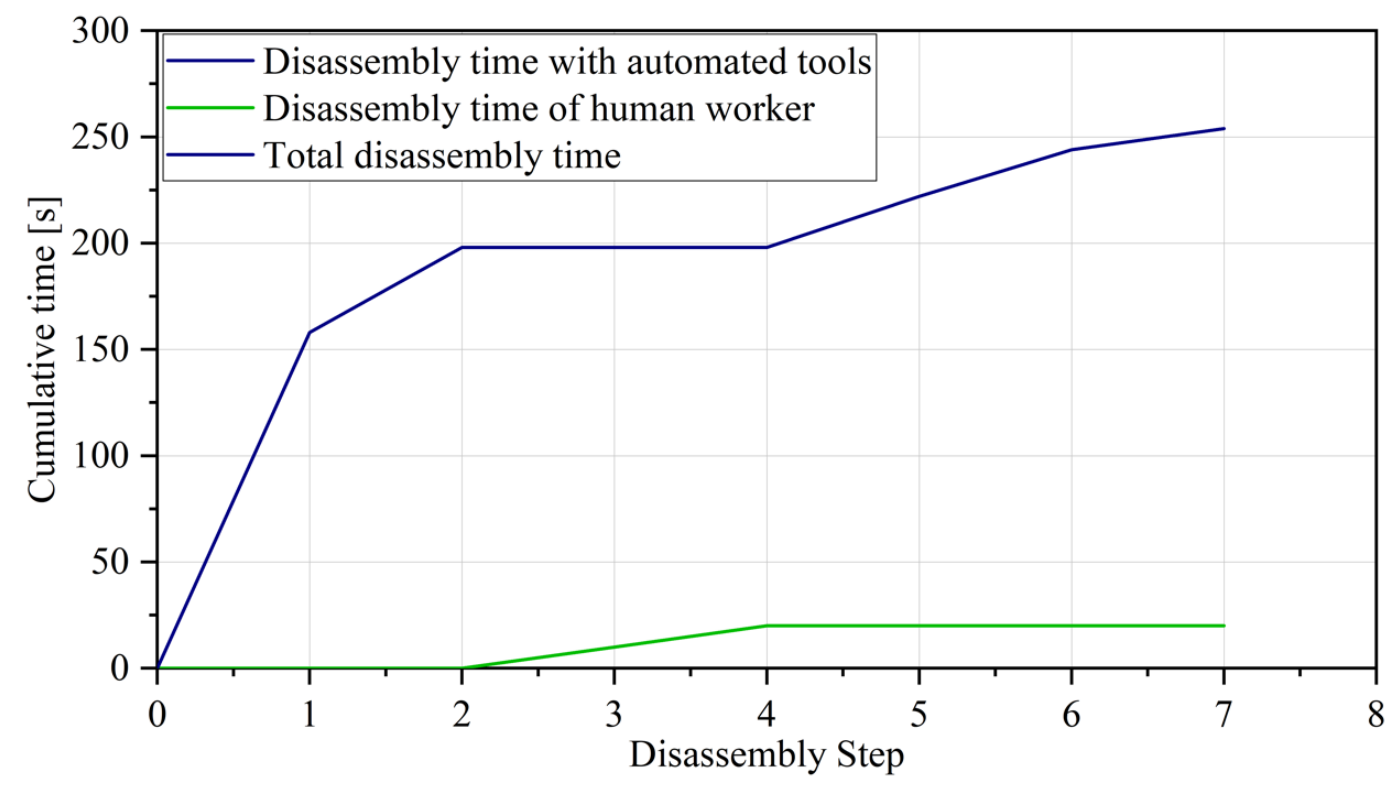

Figure 43: Disassembly time for disassembly of modeled BEV battery with two collaborative UR10e robots

\subsubsection{Calculation of Disassembly Times for example BEV Battery}

Based on the results of the simulation, an Excel calculator for the disassembly time of the 2017 Chevrolet Bolt battery was developed. Approximations similar to these used for the simulation are taken for the time each disassembly operation takes. Therefore, times for accelerating, braking, travelling, fastener removal, tool changes, grabbing operations of small, medium and large sized parts and difficult lifting operations for the 
robot and the human worker are approximated. Based on the assessment done in section 3.2.3 for each disassembly task, it is decided if it is assigned to the human or robot. All disassembly steps with a positive TAA and NA scoring are automated. The calculator provides the manual and automated disassembly time of each of the 46 disassembly steps. Figure 44 provides the results of that calculation. For example, disassembly step D1, the "Bolts around the Top Cover" scores 40 on NA and 90 on TAA. There are 56 fasteners and no tool changes or grabbing operations. The average distance between two fasteners was approximated to $100 \mathrm{~mm}$. So, in total a distance of $5600 \mathrm{~mm}$ has to be traveled. It was assumed, that the tool has to go to each bolt twice, one time for unscrewing and one time for collecting the bolt. So, there have to be two times 56 accelerating and braking processes and a travelling distance of $100 \mathrm{~mm}$. For braking and accelerating of the gantry robot a delay of 0.5 seconds and a travelling speed of $2 \mathrm{~m} / \mathrm{s}$ was assumed. That sums up to a total disassembly time of 229.6 seconds for that first disassembly task. The complete disassembly of the BEV battery with the gantry robot consumes approximately a time of about 38 minutes, while about $40 \%$ of it has to be done manually by a human worker. Results from more detailed simulations or experiments with layouts can be fed to this calculator to make it more accurate.

The same calculations were performed for the layout with the two collaborative robots. The parameters were assumed similarly to those for the gantry robot layout, but adjustments were made in order to represent the different performance of collaborative robots. For example, a longer time for unscrewing, or slower motions were assumed. Also, it was taken considered that some of the disassembly steps (e.g. D39: Battery Section Lifting) have to performed manually and take longer. But the disassembly time 
with two collaborative robots is shortened, because both robots can work in parallel on the same disassembly task, or different disassembly tasks could be done at the same time, for example if the human worker disassembles in parallel to the robots. For example, the 56 bolts of D1 are shared by both robots and each only unfastens 23 bolts. As another example one robot can perform D3, the unscrewing of the bolts for the electrical connector housing, while the human worker lifts the top cover (D2). As a result the total disassembly time is not as much as that of the human worker and that of the robots added, because of the parallel working. Still, with those assumptions, disassembly takes about 41 minutes (Figure 45). So again, the layout with a Cartesian gantry robot performs slightly better on total disassembly time. Also, the time the human worker needs for his disassembly tasks is significantly lower for the gantry layout. That promises lower operating costs for this layout due to savings in the high costs for human labor.

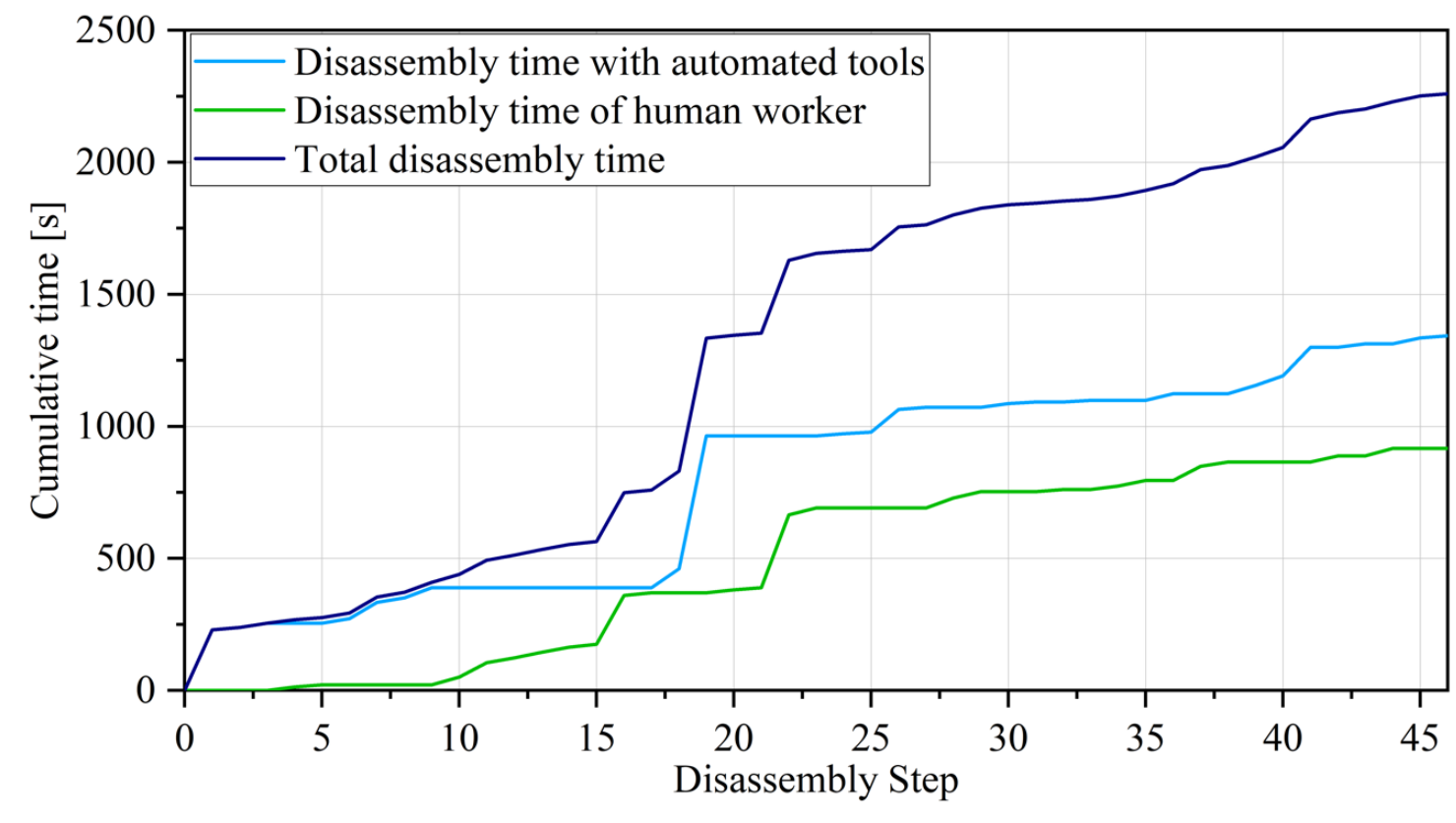

Figure 44: Calculated disassembly time for the 46 disassembly steps of the 2017 Chevrolet Bolt Battery with a gantry robot 


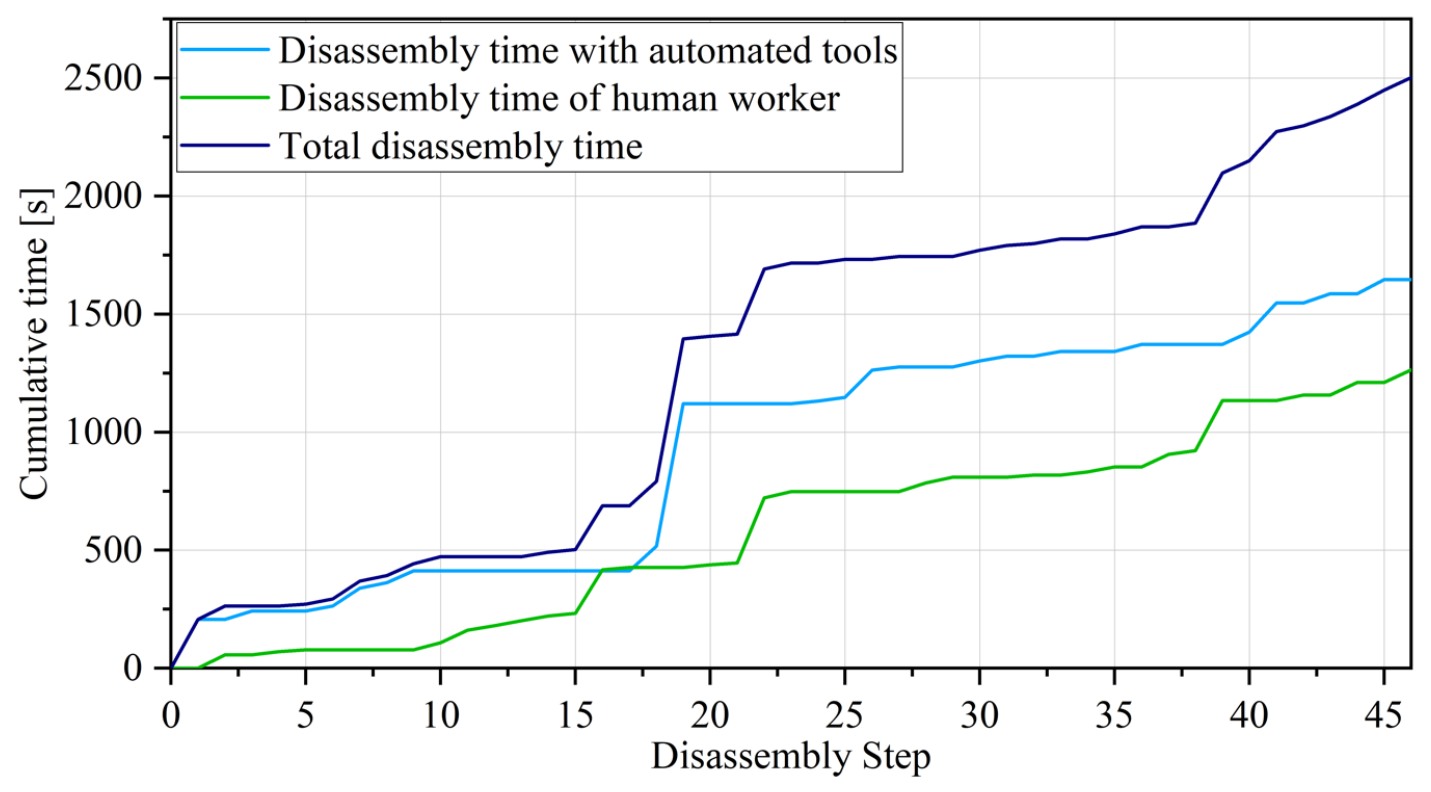

Figure 45: Calculated disassembly time for the 46 disassembly steps of the 2017 Chevrolet Bolt Battery with two collaborative robots

\subsubsection{Comparison by other Factors}

The two layouts can also be compared by the needed size in a disassembly factory, investment costs or operating costs.

The needed space excluding the conveyors or additional spaces for the collection of parts is compared. The layout with one large Gantry robot needs at least the size of the gantry robot. That is 2.7 meters in $\mathrm{x}$-direction, 3 meters in $\mathrm{y}$-direction and a height of about 4 meters, if the end effector is in its highest position above the ground. The layout with two collaborative UR10e robots needs about 3.5 meters in width between the start of the two conveyors. The linear slides have a length of 3 meters and if the robots take their arms straight up, height of a least two meters would be necessary for the displayed design, not including the proposed crane for lifting the Battery Sections. It can be stated that there are no major differences in space requirements. 
For the cost comparison this study can only perform rough assumptions. Definitely a large Gantry robot is more expensive than a smaller collaborative UR10e robot. But, two UR10e robots and additionally linear slides would be necessary. Also, there is the need for an additional lifting tool, for example a manual crane. It is assumed, that the layout with a large gantry robot has still slightly higher investment costs than a layout with two collaborative robots, also bearing in mind the difficulties of installing a large and heavy gantry robot in a disassembly factory.

The operating costs include electricity, maintenance and as the most prominent costs, human labor. As noticed from the simulation, the two collaborative robots are not able to perform as many tasks automatically, as the gantry robot can do. So, a human worker would be needed for operating the manual crane for lifting the top cover or the battery modules. A break-even point for the amount of disassembled batteries could be calculated, because with a higher number of disassembled batteries the operating costs get more important than the initial investment costs. From that rough approximations it can be concluded that the gantry layout performs slightly better as recent studies [2], [14] show, there are predictions for high numbers of EOL EV batteries, so operating costs will be more important than initial investments for a high workload of the disassembly plant.

\subsubsection{Conclusions of Layout Comparison}

In this section both layouts will be compared in general and the findings from the previous sections will be combined (see Table 13). At first, the gantry robot moves much faster, so it disassembles single parts and fasteners much faster than the collaborative UR10e robots do. The advantage of the layout with the two collaborative robots is that the human worker can work in parallel to the robots or can even teach the robots the positions 
of parts and fasteners (discussed earlier). The two robots can also work in parallel and save disassembly time that way. The main disadvantage of collaborative UR10e robots is their low payload of $10 \mathrm{~kg}$ [98]. Even if those two robots would collaborate, they could not lift the Battery Sections of the 2017 Chevrolet Bolt. The range of the UR10e robots is enough to cover the BEV battery if there is one robot positioned on a linear slide on each side of the battery. Another disadvantage of a layout with two smaller robots is that all tools have to be provided twice, which raises the investment costs. Also, the disassembled objects are placed on conveyors on each side. That requires the need for a more complicated sorting of the disassembled parts and fasteners.

Table 13: Comparison of two disassembly cell layouts

\begin{tabular}{|c|c|c|}
\hline Layout: & One Gantry robot & Two Collaborative UR10e robots \\
\hline Advantages & $\begin{array}{ll}\text { - } & \text { Faster moving robot } \\
\text { - } & \text { High payload of robot } \\
\text { - } & \text { All disassembly steps can be } \\
& \text { performed } \\
\text { - } & \text { Less disassembly time in total } \\
\text { - } & \text { Lower operating costs } \\
& \text { assumed (less human labor) }\end{array}$ & $\begin{array}{ll}\text { - } & \text { Human-robot collaboration is } \\
\text { possible } \\
\text { - } & \text { Higher safety for human } \\
\text { worker } \\
\text { - } \quad \text { Two robots and human can } \\
\text { work in parallel } \\
\text { - Locations can be taught to } \\
\text { robot physically } \\
\text { - Slightly lower initial } \\
\text { investment costs assumed }\end{array}$ \\
\hline Disadvantages & $\begin{array}{l}\text { - } \begin{array}{l}\text { No human-robot collaboration } \\
\text { (or further modifications }\end{array} \\
\text { necessary) } \\
\text { - } \quad \text { The robot cannot work when } \\
\text { humans enter the disassembly } \\
\text { area } \\
\text { - Fastener positions have to be } \\
\text { detected by a vision system, or } \\
\text { coordinates must be provided } \\
\text { by human worker }\end{array}$ & $\begin{array}{ll}\text { - } & \text { Not every disassembly step } \\
\text { can be performed (Battery } \\
\text { Sections) } \\
\text { - } \quad \text { Slower motions } \\
\text { - } & \text { Difficulty to coordinate two } \\
\text { robots simultaneously } \\
\text { - } & \text { All tools for the robots have } \\
\text { to be provided twice } \\
\text { - }\end{array}$ \\
\hline
\end{tabular}


From the comparisons it can be concluded, that a layout with one large Cartesian gantry robot is more suitable for the disassembly of large BEV batteries. That is a main difference to the investigations on HEV batteries [6], [10], [86] that suggested a small collaborative robot for the disassembly. That different conclusion refers to the larger size of the BEV and the higher weights of certain parts, especially the Battery Sections that cannot be handled by small collaborative robots.

\subsection{Further suggestions for EV Battery Disassembly}

In this section further suggestions for EV battery disassembly will be discussed. Also, the combination of the treatment of BEV and HEV/PHEV batteries is an interesting problem. The sorting and treatment of disassembled parts will also be touched briefly.

The simulation was extended for including a simplified treatment of disassembled parts (the system is shown in Figure 46). The gantry robot sorts the disassembled fasteners to the left conveyor. Those fasteners can be recycled as metallic trash. All other parts that are not disassembled by the human worker are put to the right, large conveyor by the gantry robot. Those parts are sorted further by another robot (see Figure 47a, b). This robot can be an articulated robot, but it needs to have a high payload because of the heavy weight of the Battery Sections or Modules. In the model, the disassembled parts are separated into three categories at first. The very large parts are sorted on a euro-pallet for further manual processing. In the simulation the top cover is taken for that. Another example of such a large part is P41, the "Coolant Plate." All other disassembled parts, mostly metal trash such as P32 "Front Bracket Driver 1", are sorted to the conveyor in the front and lastly into a trash collection for metals (Figure 47d). The most important parts, the Battery Sections, are sorted to the rear conveyor. There the Battery Sections are 
transported to the second disassembly worktable. At this second disassembly worktable (Figure 47c), the Battery Sections (or Modules) are disassembled down to cell level. That disassembly can be done with human robot collaborations, because the Battery Sections themselves are heavy but the parts that are disassembled from them are not heavy. The disassembly of the Battery Sections of the 2017 Chevrolet Bolt was not analyzed in detail in this study because the given video material [21], [22]. Only the disassembly of the Battery Modules of the Audi Q5 hybrid was assessed. The study by Wegener et al. [6] states that disassembly of the Battery Modules also needs to be done collaborative by a human and a robot.

At such a station for collaborative disassembly, in parallel complete HEV/ PHEV batteries can be disassembled because those have a similar weight and size as the Battery Modules of large BEV batteries. With that combination the time of disassembly steps until the Gantry robot reaches the Battery modules can be used for HEV/PHEV battery disassembly and a higher workload of the factory can be achieved. The station could be designed similar to that of Gerbers et al. [86]

Another idea could be a combination of both layouts. A gantry robot could be used for easy automatable repetitive tasks such as the screws around the top cover, while a mobile collaborative robot could carry out the more difficult tasks that do not include large and heavy parts such as cutting the harnesses. For such operations the gantry could go back to a safe default position while human worker enters the work area and teaches the collaborative robot. For tasks the collaborative robot already learned it could work in parallel with the Gantry robot in order to achieve a faster disassembly time. 


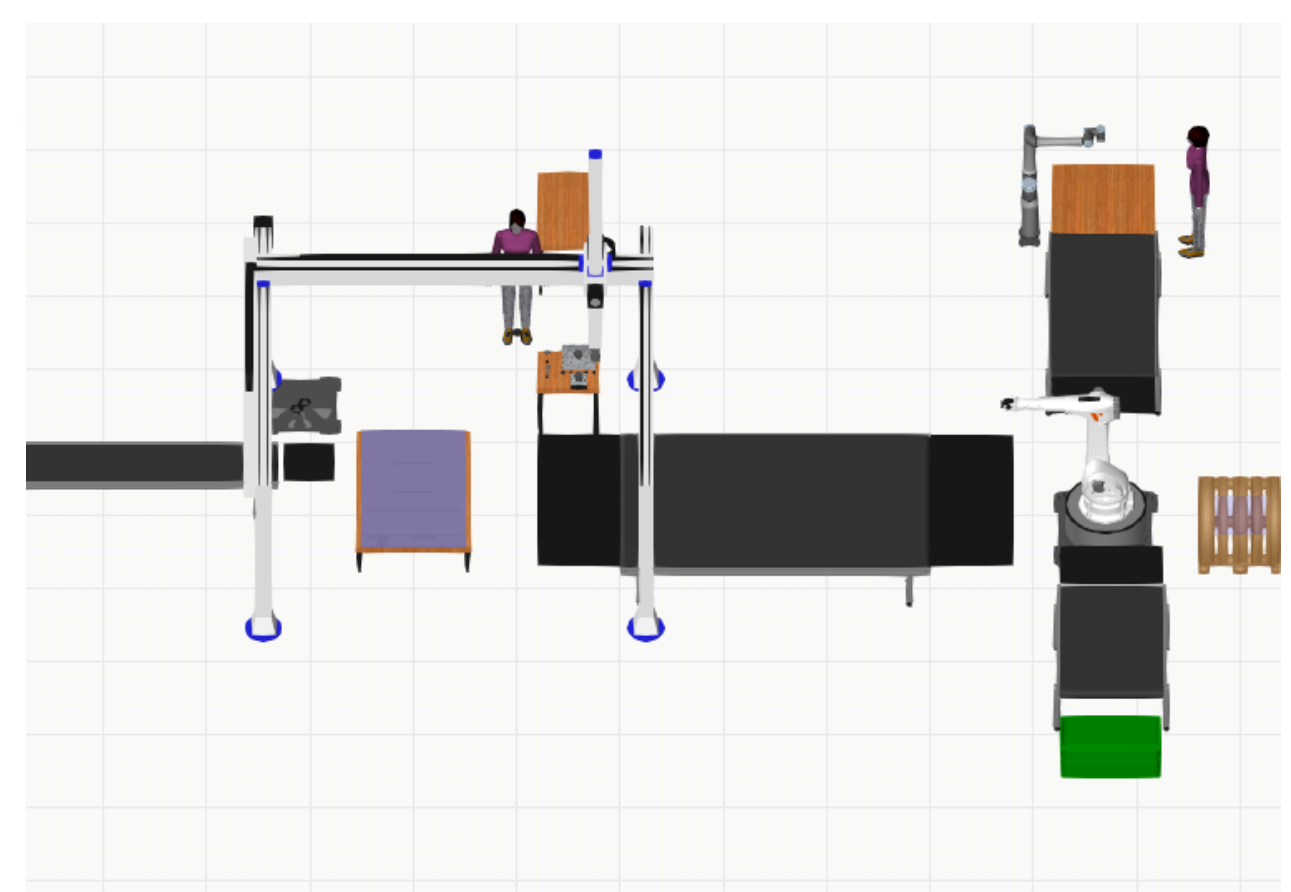

Figure 46: Extended Gantry robot layout with a suggestion for the further treatment of the disassembled parts

a)

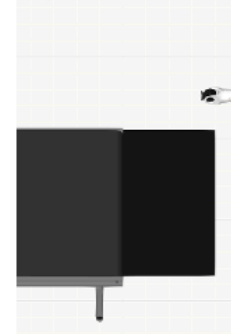

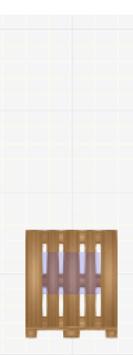

c)

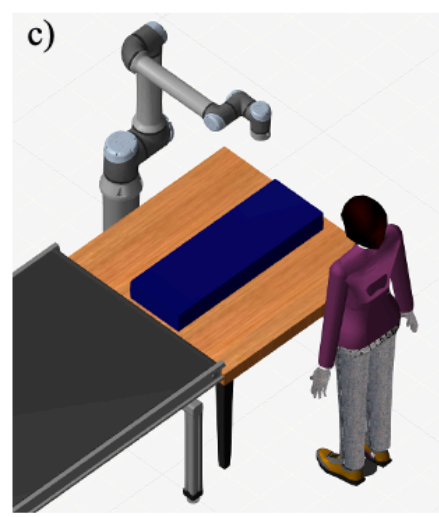

b)

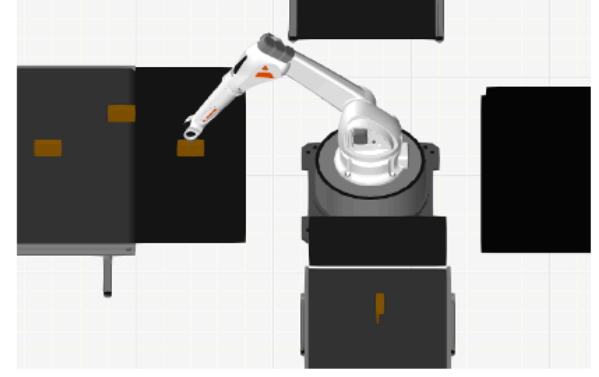

d)

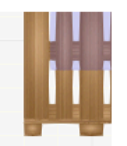

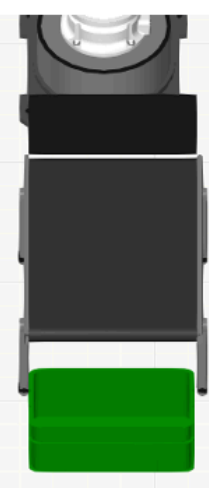

Figure 47:a) The sorting robot, b) Sorting Brackets on the Conveyor for metal trash, c) Collaborative workstation for Battery Module/Section disassembly d) Metal trash collection 


\section{Summary, Conclusions and Future Work}

A brief summary will be given before the main observations and findings will be discussed. Finally, an outlook with ideas for future research will be given.

The first goal of this thesis was to obtain an overview on recent developments in the recycling and especially the disassembly of electric vehicle batteries. Some recent studies investigated the disassembly of smaller hybrid electric vehicle batteries in details. This study aimed for finding a suitable way for the disassembly of large BEV batteries. Not only recent studies on EV battery recycling and disassembly were reviewed, but also studies on the recycling of electronic waste were considered, because there is a longer record in research and experience in this field compared to the relatively young research area of EV battery disassembly. Also, tools and techniques developed for e-waste disassembly can be adjusted for EV battery disassembly. At first the research on EV battery design and recycling indicated a large variety in the design of EV batteries that makes it more difficult to use robots in disassembly because the disassembly is less predictive. That suggests a human-robot collaboration approach. Some of the disassembly tasks are automated and some are performed by a human worker. An assessment weather which disassembly tasks should and could be automated was already performed for hybrid electric vehicle batteries. Based on that an assessment in technical ability to automate (TAA) and necessity to automate (NA) for a battery electric vehicle using as an example the 2017 Chevrolet Bolt. For that assessment a product analysis was first carried out in order to obtain a disassembly graph. Based on the disassembly graph a disassembly sequence with 46 disassembly steps was developed. A criteria catalogue was created for assessing each of the 46 disassembly steps in TAA an NA. With the same criteria 
catalogue a hybrid vehicle battery was assessed and the results for the HEV battery and the BEV battery were compared to an earlier study with a similar assessment on EV batteries. Following that a simplified model of a BEV battery with the most important parts was developed and disassembly was simulated. Two different designs for EV battery disassembly were compared. One design used one large gantry robot, while the other design used two collaborative $U R 10 e$ robots mounted on linear slides on each side of the battery.

The literature review showed that human-robot collaboration is a promising concept for more efficient disassembly of products where high uncertainties exist about their design and their condition at the end of their life. Vision systems are necessary for recognition of parts and fasteners and still lack in accuracy, but neural networks seem to be very useful for more reliable vision systems. There is a large variety of approaches for automated tools, such as cutting tools, prying tools, different grabbing tools or automated screwdrivers. In several studies in the economics of EV battery disassembly, it was stated that there will be a high amount of EOL batteries in future and high prices for raw materials ensure profitably of EV battery recycling.

The identification of all parts and fasteners of the 2017 Chevrolet Bolt battery and the development of the disassembly graph showed the complexity of EV batteries. All constraints between the 374 fasteners and the 76 parts of the EV battery were visualized. For generating an optimized disassembly sequence, based on the graph, similar disassembly operations such as different unscrewing tasks that were possible to do at one time were combined for reducing the number of tool changes. The number of disassembly steps was reduced to 46 single steps. The assessment of the disassembly steps resulted in a 
scattering of results in TAA and NA. Some steps strongly need automation and there are promising concepts for automating those, while some steps do not need automation or are very challenging to automate with current technologies. That underlines the need for human-robot-collaboration. The comparison to the results for the HEV battery pointed out major differences in assessment resulting in requirements for a disassembly work cell. Due to its size the BEV battery got more repetitive tasks which create a strong need for automation in order to prevent expensive human wok time, while the complex design makes automation of certain operation more difficult. Also, single parts such as the battery modules or the top cover of BEV batteries are much larger and heavier that the corresponding parts in HEV/PHEV batteries.

The comparison of the two disassembly layouts for BEV batteries indicated that a large Cartesian gantry robot seems more suitable for large and heavy BEV batteries. Such robots are not as suitable for human-robot collaboration due to the risk of collision, but small and collaborative robots such as the UR10e cannot handle the large and heavy parts of BEV batteries. The comparison of disassembly time for the simplified BEV model showed, that the gantry robot layout is slightly faster. Based on the simulation, calculations for all 46 disassembly steps for the combined disassembly time of the robot and human worker were performed. Again, an advantage in disassembly time for the gantry robot layout was indicated. Finally, an approach for the further processing of disassembled parts suggests collecting different kinds of parts while the battery modules are delivered to another workstation where they are disassembled together with modules of PHEV/HEV batteries by a human and a collaborative robot. 
Future research promises more accurate and intelligent vision systems using neural networks Also the usage of cloud computing can lead to international exchange of collected data about design and optimal disassembly sequences. So, once an EV battery model is disassembled a few times and the robots are taught by skilled human workers, the system can work more independently each time for one or a similar battery design even in other disassembly plants. In addition, further developments of tools, especially grabbing tools, or specialized lifting tools for heavy parts and reliable automated screwdrivers are necessary. 


\section{Appendices}

The details of the conducted assessments and simulations will be listed in the following sections.

\subsection{Assessment of EV Batteries}

In this part of the appendix the detailed results of the assessments of the EV batteries will be listed. At first the results for the assessment of the 2017 Chevrolet Bolt battery will be listed, after that the detailed assessments for each criterion of every one of the 46 disassembly steps will be listed. The same detailed information will be given for the Audi Q5 hybrid battery.

\subsubsection{TAA and NA Results for BEV Battery}

Assessment in TAA and NA for all 46 disassembly steps of the 2017 Chevrolet Bolt disassembly can be seen in the following table:

\begin{tabular}{|c|l|l|l|l|}
\hline Step & $\begin{array}{l}\text { Involved Parts or } \\
\text { Fasteners }\end{array}$ & Description & NA & TAA \\
\hline D1 & F1-56 & Bolts for Top Cover & 40 & 90 \\
\hline D2 & P1 & Lifting of Top Cover & 10 & 30 \\
\hline D3 & F57-60 & Bolts for Electrical Connector & 30 & 70 \\
\hline D4 & P2 & Electrical Connector & -20 & 50 \\
\hline D5 & P3 & Seal & -30 & 0 \\
\hline D6 & F74, F77, F80, F81 & Covers for Busbars (Front) & 20 & 20 \\
\hline D7 & F61-63, F66-69, F75-76, & $\begin{array}{l}\text { Nuts/ Screws for Busbars (Front) and Relay } \\
\text { Cover }\end{array}$ & 60 & 30 \\
\hline D8 & P4, P8-10 & Relay Cover, Busbars & 30 & 50 \\
\hline D9 & F70-73, F84-88 & Nuts, Screws below Relay Cover & 50 & 30 \\
\hline
\end{tabular}




\begin{tabular}{|c|c|c|c|c|}
\hline D10 & P5-7, P11 & $\begin{array}{l}\text { Grabbing of Relay Center, Terminals, AC- } \\
\text { Charger }\end{array}$ & 30 & -40 \\
\hline D11 & F105-108 & Connectors and Nut for Coolant Hoses & -10 & -50 \\
\hline D12 & F64-65 & Big Nuts for Coolant Hoses & -20 & -30 \\
\hline D13 & $\mathrm{P} 12, \mathrm{P} 13$ & Grabbing of Coolant Hoses & -40 & 50 \\
\hline D14 & F357-360 & Nuts for Electrical Connector & 0 & 60 \\
\hline D15 & P62 & Electrical Connector & -40 & 20 \\
\hline D16 & F89-100, F110-139, & Assurance Clips, Temp. Sensors, BECM clips & 60 & -20 \\
\hline D17 & P14 & BECM & 0 & 80 \\
\hline D18 & $\begin{array}{l}\text { F101-102, F189-190, } \\
\text { F193-194, F197-198, } \\
\text { F201-202, F205-206 } \\
\text { F209-210, F213-214 }\end{array}$ & Covers for Busbars, High Voltage Disconnect & 50 & 20 \\
\hline D19 & $\begin{array}{l}\text { F103-104, F109, } \\
\text { F140-143, F191-192, } \\
\text { F195-196, F199-200, } \\
\text { F203-204, F207-208, } \\
\text { F211-212, F215-280, } \\
\text { F290-326 }\end{array}$ & $\begin{array}{l}\text { Nuts, Bolts and Screws for Busbars and } \\
\text { Brackets, High Voltage Disconnect and Battery } \\
\text { Sections }\end{array}$ & 50 & 50 \\
\hline D20 & $\mathrm{P} 15$ & High Voltage Disconnect & 0 & 60 \\
\hline D21 & P16 & Low Voltage Harness & 0 & -20 \\
\hline D22 & F144-188 & Clips and Temp. Sensors for HV Sense Lines & 50 & -20 \\
\hline D23 & P17, P18 & HV Sense Lines & 20 & -20 \\
\hline D24 & P26-27 & Rear Brackets & 0 & 60 \\
\hline D25 & $\mathrm{P} 28$ & Cover Battery Section 5 & 20 & 30 \\
\hline D26 & $\begin{array}{l}\text { P19-25, P29-30, P32-35, } \\
\text { P37-38, P51-52, P53-54 }\end{array}$ & Busbars and Brackets & 50 & 30 \\
\hline $\mathrm{D} 27$ & P40-41 & HT Mats Battery Section 5 & 10 & 10 \\
\hline D28 & F284-289 & Hose Champs & 10 & 0 \\
\hline D29 & P42-44 & Hoses & -30 & 10 \\
\hline D30 & F281-283 & Nuts for Coolant Plate & 30 & 80 \\
\hline D31 & $\mathrm{P} 45$ & Coolant Plate Section 5 & 30 & 10 \\
\hline D32 & $\mathrm{P} 46$ & Insulating Pad Battery Section 5 & 0 & 20 \\
\hline D33 & P47 & Cover Battery Section 4 & 20 & 30 \\
\hline D34 & F327-328 & Clips HV Harness Battery Section 4 & 0 & -10 \\
\hline D35 & $\mathrm{P} 48-49$ & Side Brackets Battery Section 4 & 0 & 60 \\
\hline
\end{tabular}




\begin{tabular}{|l|l|l|l|l|}
\hline D36 & F337-342 & Bolts Battery Section 4 & 40 & 70 \\
\hline D37 & F329-336 & Retainers, Position Assurance Battery Section 4 & 30 & 0 \\
\hline D38 & P55-56 & HV Monitoring Circuit Battery Section 4 & 0 & 60 \\
\hline D39 & P36, P39, P50, P57 & Battery Sections 1-4 Lifting & 90 & 30 \\
\hline D40 & P63-70 & Heat Transfer Mats & 10 & 10 \\
\hline D41 & $\begin{array}{l}\text { F343-356, F361-371, } \\
\text { F375 }\end{array}$ & Bolts for Braces, Coolant Plate & 10 & 80 \\
\hline D42 & P58-61 & Braces & -30 & 70 \\
\hline D43 & F372-374 & Bolts for Coolant Plate & -20 & 80 \\
\hline D44 & P71 & Coolant Plate & 0 & 10 \\
\hline D45 & P72-75 & Insulating Pads & -10 & 20 \\
\hline D46 & P76 & Handling of Battery Tray & 40 & 40 \\
\hline
\end{tabular}




\subsubsection{Detailed Assessments of each Disassembly step for BEV Battery}

\section{D1: Bolts for Top Cover, F1-56}

56 Bolts, 50 Bolts around Top Cover, 6 Bolts around High Voltage Disconnect

\begin{tabular}{|l|l|l|}
\hline Criteria & Comments & Scoring \\
\hline N1 & $\begin{array}{l}\text { Move, position, unscrew, move, grasp, bring, release for every bolt: 7*56= } \\
392\end{array}$ & 2 \\
\hline N2 & Assume 5s for every bolt, 280s in total & 2 \\
\hline N3 & No high voltage or chemical dangers at that point, only sharp edges possible & -1 \\
\hline N4 & Very low weights, just bolts, some bending to reach bolts & -1 \\
\hline N5 & $\begin{array}{l}\text { Necessary to reach cells because no other possibilities to come to cells/modules } \\
\text { and most other parts }\end{array}$ & 2 \\
\hline T1 & Just simple bolts, no tool changing necessary & 2 \\
\hline T2 & Open access from the top & 2 \\
\hline T3 & Detection from the top, different color, relatively large bolts & 2 \\
\hline T4 & Some choices: e.g. Li et al. [26] & 1 \\
\hline T5 & Collection of Bolts, sorting in Screw/Bolt/ Nut collection & 2 \\
\hline
\end{tabular}

$\mathrm{NA}=40, \mathrm{TAA}=90$

\section{D2: Lifting of Top Cover, P1}

1 large Cover

\begin{tabular}{|l|l|l|}
\hline Criteria & Comments & Scoring \\
\hline N1 & Move, grasp, bring, release (two workers needed) $=8$ & -1 \\
\hline N2 & Strongly depended on distance, assume 20s * 2 workers = 40s & 1 \\
\hline N3 & No high voltage or chemical dangers at that point, only sharp edges possible & -1 \\
\hline N4 & Assume 10-15kg, no bending needed & 0 \\
\hline N5 & $\begin{array}{l}\text { Necessary to reach cells because no other possibilities to come to cells/modules } \\
\text { and most other parts }\end{array}$ & 2 \\
\hline T1 & Translational movement and grabbing of larger part & 0 \\
\hline T2 & Completely open access for end effector, but side access & 1 \\
\hline T3 & $\begin{array}{l}\text { Detection good possible, open view, just approaching from sides, need force } \\
\text { tactile robot }\end{array}$ & 1 \\
\hline T4 & Some choices: e.g. Borràs et al. [83] & 1 \\
\hline T5 & Handling of large part, just one type of material, possibly crane lifting & 0 \\
\hline
\end{tabular}

$\mathrm{NA}=10, \mathrm{TAA}=30$ 


\section{D3: Bolts for Electrical Connector, F57-60}

4 Bolts, open access from the Side

\begin{tabular}{|l|l|l|}
\hline Criteria & Comments & Scoring \\
\hline N1 & Move, position, unscrew, move, grasp, bring, release for every bolt: $7 * 4=28$ & 1 \\
\hline N2 & Assume 5s for every bolt = 20s & 0 \\
\hline N3 & Low Danger of HV & 0 \\
\hline N4 & Low weight of bolts, but strong bending, some twisting needed & 0 \\
\hline N5 & Necessary to reach Battery Modules and most other valuable parts & 2 \\
\hline T1 & Standard movements for unscrewing & 2 \\
\hline T2 & Side access & 1 \\
\hline T3 & Side detection, but good contrast and relatively large bolts & 1 \\
\hline T4 & Some choices: e.g. Li et al. [26] & 1 \\
\hline T5 & Simple collection of bolts & 2 \\
\hline
\end{tabular}

$\mathrm{NA}=30, \mathrm{TAA}=70$

\section{D4: Electrical Connector, P2}

One Electrical Connector (medium size part)

\begin{tabular}{|l|l|l|}
\hline Criteria & Comments & Scoring \\
\hline N1 & Move, grasp, bring, release $=4$ & -1 \\
\hline N2 & Assume 10s & -1 \\
\hline N3 & Low danger of HV & 0 \\
\hline N4 & Low weight, smaller than 5kg & -2 \\
\hline N5 & Necessary to reach Battery Modules and most other valuable parts & 2 \\
\hline T1 & Simple Grabbing & 2 \\
\hline T2 & Nearly completely open, depends on end effector & 1 \\
\hline T3 & Partly hidden from top, but good contrast, medium size part & 1 \\
\hline T4 & Some choices: e.g. Borràs et al. [83] & 1 \\
\hline T5 & Different materials medium size part & 0 \\
\hline
\end{tabular}

$\mathrm{NA}=-20, \mathrm{TAA}=50$ 


\section{D5: Seal, P3}

1 Seal, maybe grabbing at different places simultaneously

\begin{tabular}{|l|l|l|}
\hline Criteria & Comments & Scoring \\
\hline N1 & Move, grasp, bring, release $* 2=8$ & -1 \\
\hline N2 & Assume 15s & 0 \\
\hline N3 & Low Danger of High Voltage & 0 \\
\hline N4 & Low weight, some bending needed & -1 \\
\hline N5 & $\begin{array}{l}\text { Not necessary to reach Battery Modules, low cost part (but could affect further } \\
\text { disassembly negatively) }\end{array}$ & -1 \\
\hline T1 & More difficult grabbing operation & 0 \\
\hline T2 & Size limitations for end effector & 1 \\
\hline T3 & More difficult detection because of bad contrast and relatively slim part & 0 \\
\hline T4 & Possible Choice: e.g. Borràs et al. [83] & -1 \\
\hline T5 & More difficult to handle, sorting in trash & 0 \\
\hline
\end{tabular}

$\mathrm{NA}=-30, \mathrm{TAA}=0$

D6: Covers for Busbars (front), F74, F77, F80, F81

4 Covers

\begin{tabular}{|l|l|l|}
\hline Criteria & Comments & Scoring \\
\hline N1 & Move, position, prying * 4=12 & 0 \\
\hline N2 & Assume 5s each = 20s & 0 \\
\hline N3 & Protection against HV danger necessary & 1 \\
\hline N4 & No weight, but some bending necessary & -1 \\
\hline N5 & Necessary for reaching Modules and most other parts & 2 \\
\hline T1 & More complex, prying, and translational & 0 \\
\hline T2 & Open access, some size limitation & 1 \\
\hline T3 & Detection of covers is more difficult but open view from top & 0 \\
\hline T4 & Only something similar: e.g. Schumacher, Jouaneh [31] & -1 \\
\hline T5 & No material handling needed, no removed part & 2 \\
\hline
\end{tabular}

$\mathrm{NA}=20, \mathrm{TAA}=20$ 
D7: Nuts/ Screws for Busbars (Front) and Relay Cover, F61-63, F66-69, F75-76, F7879, F82-83

5 Screws, 4 Bolts, 4 Nuts

\begin{tabular}{|l|l|l|}
\hline Criteria & Comments & Scoring \\
\hline N1 & 7 motions for each fastener $* 13=91$ & 2 \\
\hline N2 & Assume 1min & 2 \\
\hline N3 & HV protection necessary & 1 \\
\hline N4 & Low weight fasteners, some bending & -1 \\
\hline N5 & Necessary for Relay Center and Modules/Cells & 2 \\
\hline T1 & $\begin{array}{l}\text { Simple standard movements, normal fasteners, but 4 Bolts from the side and } \\
\text { two tool changings }\end{array}$ & 0 \\
\hline T2 & Nearly open access & 1 \\
\hline T3 & More difficult detection: Side, small screws & -1 \\
\hline T4 & Some existing choices & 1 \\
\hline T5 & Simple handling of fasteners & 2 \\
\hline
\end{tabular}

$\mathrm{NA}=60, \mathrm{TAA}=30$

\section{D8: Relay Cover, Busbars, P4, P8-10}

1 medium size Cover, 3 Busbars

\begin{tabular}{|l|l|l|}
\hline Criteria & Comments & Scoring \\
\hline N1 & Move, grasp, bring, release $* 4=16$ & 0 \\
\hline N2 & Assume 30s & 1 \\
\hline N3 & HV protection necessary & 1 \\
\hline N4 & Low weight parts, some bending & -1 \\
\hline N5 & Necessary for Relay Center and Modules/Cells & 2 \\
\hline T1 & Grabbing, a bit more difficult & 1 \\
\hline T2 & Some size limitations & 1 \\
\hline T3 & Very good color contrast, good detection, relatively large parts & 2 \\
\hline T4 & Some choices & 1 \\
\hline T5 & More than one type of material, parts are more difficult to handle & 0 \\
\hline
\end{tabular}

$\mathrm{NA}=30, \mathrm{TAA}=50$ 
D9: Nuts, Screws below Relay Cover, F70-73, F84-88

7 Nuts, 2 Screws

\begin{tabular}{|l|l|l|}
\hline Criteria & Comments & Scoring \\
\hline N1 & $7 * 7=49$ & 2 \\
\hline N2 & Assume 30s & 1 \\
\hline N3 & HV protection needed & 1 \\
\hline N4 & Low weight fasteners, some bending & -1 \\
\hline N5 & Necessary for Relay Center and Modules/Cells & 2 \\
\hline T1 & Simple rotational/translational unscrewing movements, one tool change needed & 1 \\
\hline T2 & Extended end-effector needed, size limitations & 0 \\
\hline T3 & Not hidden, but very small screws, difficult to find, shadows possible & -1 \\
\hline T4 & Some choices & 1 \\
\hline T5 & Simple fastener collection & 2 \\
\hline
\end{tabular}

$\mathrm{NA}=50, \mathrm{TAA}=30$

D10: Grabbing of Relay Center, Terminals, AC-Charger, P5-7, P11

1 Relay Center, 1 AC-Charger, 2 Electrical Terminals

\begin{tabular}{|l|l|l|}
\hline Criteria & Comments & Scoring \\
\hline N1 & $\begin{array}{l}\text { Move, grasp, bring, release * 5= 20 (two hands for Relay Center and in general } \\
\text { more difficult) }\end{array}$ & 0 \\
\hline N2 & Assume 30s (more difficult parts to grab) & 0 \\
\hline N3 & HV protection needed & 1 \\
\hline N4 & Weight should be lower than 5kg, but bending and twisting is necessary & 0 \\
\hline N5 & Necessary to get cells and Relay Center & 2 \\
\hline T1 & $\begin{array}{l}\text { More complicated grabbing operation, possibly different grabbers for different } \\
\text { parts needed }\end{array}$ & -1 \\
\hline T2 & Extended and flexible/small end-effector needed & -1 \\
\hline T3 & Terminals are partly hidden; parts are in general difficult to detect & -1 \\
\hline T4 & Some choices for grabber/lifting tools, but unsure, if those would work here & 0 \\
\hline T5 & $\begin{array}{l}\text { Different materials inside parts, need to be stored for further recycling, lifting } \\
\text { tool for relay Center needed }\end{array}$ & -1 \\
\hline
\end{tabular}

$\mathrm{NA}=30, \mathrm{TAA}=-40$ 
D11: Connectors and Nut for Coolant Hoses, F105-108

3 Connectors, 1 Nut

\begin{tabular}{|l|l|l|}
\hline Criteria & Comments & Scoring \\
\hline N1 & Move, position, unclip, grasp, bring, release * 3+7=25 & 1 \\
\hline N2 & Assume 20s (unsure about fastness of unclipping) & 0 \\
\hline N3 & HV protection necessary & 1 \\
\hline N4 & Low weight, some bending & -1 \\
\hline N5 & Not needed to reach cells, only material sorting, low cost & -2 \\
\hline T1 & At least one tool change and more complex unclipping & -1 \\
\hline T2 & Extended and small/flexible end-effector needed & -1 \\
\hline T3 & Small connectors/nuts, bad contrast and partly hidden & -1 \\
\hline T4 & $\begin{array}{l}\text { Maybe choices for grabbers and cutters could be used, but nothing special for } \\
\text { this, nut could be done by automated nutrunner }\end{array}$ & -1 \\
\hline T5 & Collecting of small trash parts, but different trash for sorting & -1 \\
\hline
\end{tabular}

$\mathrm{NA}=-10, \mathrm{TAA}=-50$

\section{D12: Big Nuts for Coolant Hoses, F64-65}

\section{Large Nuts}

\begin{tabular}{|l|l|l|}
\hline Criteria & Comments & Scoring \\
\hline N1 & Assume 15 movements & 0 \\
\hline N2 & Assume 20s & 0 \\
\hline N3 & Low HV danger & 0 \\
\hline N4 & Low weight, some bending & -1 \\
\hline N5 & Not necessary to reach Cells, just material sorting for further recycling & -1 \\
\hline T1 & Very complex, much force needed & -2 \\
\hline T2 & Maybe extended end effector needed & 0 \\
\hline T3 & Partly hidden & 0 \\
\hline T4 & No proposed concept, uncertainity about possible automation & -2 \\
\hline T5 & Collection of metal trash, but more difficult to handle than normal screw & 1 \\
\hline
\end{tabular}

$\mathrm{NA}=-20, \mathrm{TAA}=-30$ 
D13: Grabbing of Coolant Hoses, P12-13

2 Coolant hoses

\begin{tabular}{|l|l|l|}
\hline Criteria & Comments & Scoring \\
\hline N1 & Move, grasp, bring, release $* 2=10$ & -1 \\
\hline N2 & Assume 10s & -1 \\
\hline N3 & Low HV danger & 0 \\
\hline N4 & Low weight, some bending & -1 \\
\hline N5 & $\begin{array}{l}\text { Not necessary for cells and other valuable parts, only sorting for further } \\
\text { recycling }\end{array}$ & -1 \\
\hline T1 & Medium difficult grabbing & 1 \\
\hline T2 & Some size limitations & 1 \\
\hline T3 & Good color difference, easy to detect & 1 \\
\hline T4 & Some existing choices & 1 \\
\hline T5 & Collecting medium size recycled parts & 1 \\
\hline
\end{tabular}

$\mathrm{NA}=-40, \mathrm{TAA}=50$

D14: Nuts for Electrical Connector, F357-360

4 Bolts

\begin{tabular}{|l|l|l|}
\hline Criteria & Comments & Scoring \\
\hline N1 & 7 movements * 4 Nuts $=28$ & 1 \\
\hline N2 & Assume 20s & 0 \\
\hline N3 & Low HV danger & 0 \\
\hline N4 & Low weight, but assuming strong bending & 0 \\
\hline N5 & Not necessary to reach cells, part itself is not very expensive, but sorting & -1 \\
\hline T1 & Simple Nuts: Rotational, Translational & 2 \\
\hline T2 & Access from side and inside & 0 \\
\hline T3 & Relatively easy detection, but detection from side needed & 1 \\
\hline T4 & Some existing choices for bolts & 1 \\
\hline T5 & Simple material handling of bolts & 2 \\
\hline
\end{tabular}

$\mathrm{NA}=0, \mathrm{TAA}=60$ 
D15: Electrical Connector, P62

1 Electrical Connector

\begin{tabular}{|l|l|l|}
\hline Criteria & Comments & Scoring \\
\hline N1 & Move, grasp, bring, release $=4$ & -1 \\
\hline N2 & Assume 10s & -1 \\
\hline N3 & Low HV danger & 0 \\
\hline N4 & Low weight, some bending & -1 \\
\hline N5 & Not necessary for Cells, not very valuable itself, but material sorting & -1 \\
\hline T1 & Medium difficulty grabbing & 0 \\
\hline T2 & Inside access, but open access, maybe partly hidden & 0 \\
\hline T3 & Relatively open view, but different color, medium size part & 0 \\
\hline T4 & Some existing choices fo rgrabbing tools & 1 \\
\hline T5 & Collecting of medium size part for further recycling & 1 \\
\hline
\end{tabular}

$\mathrm{NA}=-40, \mathrm{TAA}=20$

D16: Assurance Clips, Temp. Sensors, BECM clips, F89-100, F110-139,

9 Assurance Clips, 3 Clips, 30 Temp. Sensors

\begin{tabular}{|l|l|l|}
\hline Criteria & Comments & Scoring \\
\hline N1 & $\begin{array}{l}\text { Move, position, unclip, grasp, bring, release or } \\
\text { Move, position, cut, grasp, bring, release * 39+3* Move, position, unclip }=\end{array}$ & 2 \\
\hline N2 & Assume 2 minutes & 2 \\
\hline N3 & HV protection needed & 1 \\
\hline N4 & Low weight, some bending & -1 \\
\hline N5 & Needed to reach Cells and BECM & 2 \\
\hline T1 & More complex and tool changing needed & -1 \\
\hline T2 & Open access, but size limitations & 1 \\
\hline T3 & Partly hidden, bad contrast, very small sizes & -1 \\
\hline T4 & Some concepts for cutting but unsure if working & -1 \\
\hline T5 & Different materials, collecting of larger trash parts, cables & 0 \\
\hline
\end{tabular}

$\mathrm{NA}=60, \mathrm{TAA}=-20$ 


\section{D17: BECM, P14}

1 BECM (medium size part)

\begin{tabular}{|l|l|l|}
\hline Criteria & Comments & Scoring \\
\hline N1 & Move, grasp, bring, release $=4$ & -1 \\
\hline N2 & Assume 10s & -1 \\
\hline N3 & HV protection necessary & 1 \\
\hline N4 & Low weight, some bending & -1 \\
\hline N5 & Necessary to reach Cells and BECM & 2 \\
\hline T1 & Relatively simple grabbing & 2 \\
\hline T2 & Open access for end-effector & 2 \\
\hline T3 & Good contrast, no shadows, easy to detect & 2 \\
\hline T4 & Some existing choices for grabbers & 1 \\
\hline T5 & Collecting of all BECMs for reuse or recycling & 1 \\
\hline
\end{tabular}

$\mathrm{NA}=0, \mathrm{TAA}=80$

D18: Covers for Busbars, High Voltage Disconnect, F101-102, F189-190, F193-194, F197-198, F201-202, F205-206 F209-210, F213-214

16 Covers

\begin{tabular}{|l|l|l|}
\hline Criteria & Comments & Scoring \\
\hline N1 & Move, position, prying $* 16=48$ & 2 \\
\hline N2 & Assume 5s each $=80 \mathrm{~s}$ & 2 \\
\hline N3 & Protection against HV danger necessary & 1 \\
\hline N4 & No weight, but some bending necessary & -1 \\
\hline N5 & Necessary for reaching Modules/Cells & 1 \\
\hline T1 & More complex, prying, and translational & 0 \\
\hline T2 & Open access, some size limitation & 1 \\
\hline T3 & Detection of covers is more difficult but open view from top & 0 \\
\hline T4 & Not sure if working: e.g. Schumacher, Jouaneh [31] & -1 \\
\hline T5 & No material handling needed, no removed part & 2 \\
\hline
\end{tabular}

$\mathrm{NA}=50, \mathrm{TAA}=20$ 
D19: Nuts, Bolts, Screws for Busbars and Brackets, High Voltage Disconnect, Battery Sections, F103-104, F109, F140-143, F191-192, F195-196, F199-200, F203204, F207-208, F211-212, F215-280, F290-326

88 Nuts, 24 Bolts, 4 Screws

\begin{tabular}{|l|l|l|}
\hline Criteria & Comments & Scoring \\
\hline N1 & $7 * 116=812$ & 2 \\
\hline N2 & Assume 5s for every Fastener $=580 \mathrm{~s}$ & 2 \\
\hline N3 & HV protection necessary & 1 \\
\hline N4 & Low weight of fasteners, some bending necessary & -1 \\
\hline N5 & Necessary to reach Cells/ Modules & 1 \\
\hline T1 & Simple unscrewing & 2 \\
\hline T2 & $\begin{array}{l}\text { Mostly good access, some size limitations, sometimes extended end-effector } \\
\text { needed }\end{array}$ & 0 \\
\hline T3 & Some are partly hidden, more difficult to detect & 0 \\
\hline T4 & Some choices & 1 \\
\hline T5 & Simple collecting of fasteners (metal) & 2 \\
\hline
\end{tabular}

$\mathrm{NA}=50, \mathrm{TAA}=50$

\section{D20: High Voltage Disconnect, P15}

1 Medium size part

\begin{tabular}{|l|l|l|}
\hline Criteria & Comments & Scoring \\
\hline N1 & 4 movements for grabbing & -1 \\
\hline N2 & Assume 10s & -1 \\
\hline N3 & HV protection necessary & 1 \\
\hline N4 & Weight $<5 \mathrm{~kg}$, but far away from body while grabbing & -1 \\
\hline N5 & Necessary to reach Modules/Cells, part itself could be reused & 2 \\
\hline T1 & Simple grabbing (but medium size) & 1 \\
\hline T2 & Open access for end-effector & 2 \\
\hline T3 & Good detection (if stored how it looks like) & 2 \\
\hline T4 & Some choices for grabber of medium size parts & 1 \\
\hline T5 & More than one type of material storage for reuse & 0 \\
\hline
\end{tabular}

$\mathrm{NA}=0, \mathrm{TAA}=60$ 
D21: Low Voltage Harness, P16

1 Harness (cable)

\begin{tabular}{|l|l|l|}
\hline Criteria & Comments & Scoring \\
\hline N1 & 4 movements for grabbing, but maybe at two different places: 8 & -1 \\
\hline N2 & Assume 20s & 0 \\
\hline N3 & HV protection needed & 1 \\
\hline N4 & Low weight, some bending/twisting & -1 \\
\hline N5 & Necessary to reach cells, but not valuable itself & 1 \\
\hline T1 & $\begin{array}{l}\text { Grabbing, but more complicated grabbing, depends, if it could be grabbed at } \\
\text { one point }\end{array}$ & -1 \\
\hline T2 & Size limitations and partly extended needed & 0 \\
\hline T3 & Difficult to detect harness due to contrast & 0 \\
\hline T4 & Some choices, but unsure if they would work & -1 \\
\hline T5 & Large part, sorting cable into trash, different materials because of sensors & 0 \\
\hline
\end{tabular}

$\mathrm{NA}=0, \mathrm{TAA}=-20$

D22: Clips and Temp. Sensors for HV Sense Lines, F144-188

27 Clips, 18 Monitoring Sensors

\begin{tabular}{|l|l|l|}
\hline Criteria & Comments & Scoring \\
\hline N1 & $\begin{array}{l}\text { Move, position, unclip, grasp, bring, release or } \\
\text { Move, position, cut, grasp, bring, release * 45 =270 }\end{array}$ & 2 \\
\hline N2 & Assume 5 min & 2 \\
\hline N3 & HV danger & 1 \\
\hline N4 & Low weight, but bending and twisting is necessary & -1 \\
\hline N5 & Necessary to reach Modules/ Cells, but low-cost part itself & 1 \\
\hline T1 & More complex unplugging or cutting & -1 \\
\hline T2 & Open access, but size limitations & 1 \\
\hline T3 & Partly hidden, bad contrast, very small sizes & -1 \\
\hline T4 & Some concepts for cutting but unsure if working & -1 \\
\hline T5 & No parts, only unplugging & 0 \\
\hline
\end{tabular}

$\mathrm{NA}=50, \mathrm{TAA}=-20$ 
D23: HV Sense Lines, P17-18

2 Sense Lines (cables)

\begin{tabular}{|l|l|l|}
\hline Criteria & Comments & Scoring \\
\hline N1 & 4 movements for grabbing, but maybe at two different places*2=16 & 0 \\
\hline N2 & Assume 30s & 1 \\
\hline N3 & HV protection needed & 1 \\
\hline N4 & Low weight, some bending/twisting & -1 \\
\hline N5 & Necessary to reach cells, but not valuable itself & 1 \\
\hline T1 & $\begin{array}{l}\text { Grabbing, but more complicated grabbing, depends, if it could be grabbed at } \\
\text { one point }\end{array}$ & -1 \\
\hline T2 & Size limitations and partly extended needed & 0 \\
\hline T3 & Difficult to detect harness due to contrast & 0 \\
\hline T4 & Some choices, but unsure if they would work & -1 \\
\hline T5 & Large part, sorting cable into trash, different materials because of sensors & 0 \\
\hline
\end{tabular}

$\mathrm{NA}=20, \mathrm{TAA}=-20$

\section{D24: Rear Brackets, P26-27}

2 Brackets

\begin{tabular}{|l|l|l|}
\hline Criteria & Comments & Scoring \\
\hline N1 & 4 movements for grabbing $* 2=8$ & -1 \\
\hline N2 & Assume 20s & 0 \\
\hline N3 & HV protection needed & 1 \\
\hline N4 & Low weight, some bending is necessary & -1 \\
\hline N5 & Necessary to reach Cells/ Modules & 1 \\
\hline T1 & Simple grabbing operation (medium size part) & 2 \\
\hline T2 & Very good access from side & 1 \\
\hline T3 & $\begin{array}{l}\text { Detection could be a little difficult because of same color, but position and } \\
\text { shape should be recognizable }\end{array}$ & 1 \\
\hline T4 & Some choices for grabber, should work on this & 1 \\
\hline T5 & Medium size part, placing into metal collection & 1 \\
\hline
\end{tabular}

$\mathrm{NA}=0, \mathrm{TAA}=60$ 


\section{D25: Cover Battery Section 5, P28}

1 large Cover

\begin{tabular}{|l|l|l|}
\hline Criteria & Comments & Scoring \\
\hline N1 & 4 movements for grabbing, but two hands would be needed: 8 & -1 \\
\hline N2 & Assume 30s & 1 \\
\hline N3 & HV protection necessary & 1 \\
\hline N4 & Approx. 5-10kg, no bending should be needed & -1 \\
\hline N5 & Large part of recyclable metal and necessary for Cells/ Modules & 2 \\
\hline T1 & Larger grabbing/ maybe lifting & 0 \\
\hline T2 & Open access from the top & 2 \\
\hline T3 & Large part, detection of part easy, maybe more difficult to find spot to grab & 1 \\
\hline T4 & Some choices for grabbing, but unsure how good working on that size & 0 \\
\hline T5 & Handling of large part, but only metal, so sorting into large metal trash & 0 \\
\hline
\end{tabular}

$\mathrm{NA}=20, \mathrm{TAA}=30$

D26: Busbars and Brackets, P19-25, P29-30, P32-35, P37-38, P51-52, P53-54

7 Bus Bars, 12 Brackets

\begin{tabular}{|l|l|l|}
\hline Criteria & Comments & Scoring \\
\hline N1 & 4 movements for grabbing $* 19=76$ & 2 \\
\hline N2 & Assume 5s for each grabbing =95s & 2 \\
\hline N3 & HV protection necessary & 1 \\
\hline N4 & Approx. 0.2-1kg each, some bending necessary or far away from body & -1 \\
\hline N5 & Necessary to reach Cells/ Modules & 1 \\
\hline T1 & $\begin{array}{l}\text { Simple grabbing operations, medium size parts, maybe some different grabbers } \\
\text { needed }\end{array}$ & 1 \\
\hline T2 & Open access for end-effectors, for some brackets partly hidden & 1 \\
\hline T3 & $\begin{array}{l}\text { Busbars have a very good contrast, good to detect, brackets are more difficult to } \\
\text { detect }\end{array}$ & 0 \\
\hline T4 & Some options & 1 \\
\hline T5 & Handling of metal brackets is easy, special collection of busbars & 0 \\
\hline
\end{tabular}

$\mathrm{NA}=50, \mathrm{TAA}=30$ 
D27: HT Mats Battery Section 5, P40-41

2 Heat Transfer Mats

\begin{tabular}{|l|l|l|}
\hline Criteria & Comments & Scoring \\
\hline N1 & Normally 4 motions for grabbing +1because of glue $* 2=10$ & -1 \\
\hline N2 & Assume 20s & 0 \\
\hline N3 & HV protection necessary & 1 \\
\hline N4 & Low weight, some bending, twisting for ungluing it & 0 \\
\hline N5 & Necessary to reach Cells/ Modules, but low-cost itself & 1 \\
\hline T1 & More complex grabbing & 0 \\
\hline T2 & Open access from top, but more difficult to get below mat & 0 \\
\hline T3 & Easy to detect because of shape and color & 2 \\
\hline T4 & Some choices, but unsure if those would work on that special case & -1 \\
\hline T5 & Sorting mats into trash (probably not reusable), maybe problems due to glue & 0 \\
\hline
\end{tabular}

$\mathrm{NA}=10, \mathrm{TAA}=10$

\section{D28: Hose Champs, F284-289}

6 Hose Champs, but cutting would be better, 3 or 6 cutting operations

\begin{tabular}{|l|l|l|}
\hline Criteria & Comments & Scoring \\
\hline N1 & Move, position, cut *3=9 & -1 \\
\hline N2 & Assume 20s & 0 \\
\hline N3 & $\begin{array}{l}\text { HV protection needed, (maybe also protection if coolant is dangerous), and } \\
\text { being careful with cutter }\end{array}$ & 2 \\
\hline N4 & Just cutting, some bending & -1 \\
\hline N5 & Necessary to reach Cells/ Modules, low-cost itself & 1 \\
\hline T1 & More complex cutting operation & 0 \\
\hline T2 & Open side access & 0 \\
\hline T3 & Not possible to detect from top, hidden: Need good detection from side & -1 \\
\hline T4 & At least one cutting choice, but unsure if working for that & -1 \\
\hline T5 & No material handling needed & 2 \\
\hline
\end{tabular}

$\mathrm{NA}=10, \mathrm{TAA}=0$ 
D29: Hoses, P42-44

3 Hoses, small parts, step is only necessary if hose champs get unfastened and not cut

\begin{tabular}{|l|l|l|}
\hline Criteria & Comments & Scoring \\
\hline N1 & $4 * 3$ motions for that grabbing operations =12 & 0 \\
\hline N2 & Assume 10s & -1 \\
\hline N3 & HV protection needed (maybe also protection if coolant is dangerous) & 1 \\
\hline N4 & Low weight parts, some bending & -1 \\
\hline N5 & Not necessary for Cells/ Modules, not reusable & -2 \\
\hline T1 & More complex grabbing from side & 0 \\
\hline T2 & Side access, some size limitations & 0 \\
\hline T3 & Detection from top not possible, need camera from side & 0 \\
\hline T4 & Some choices, should work, but unsure & 0 \\
\hline T5 & Collection of trash & 1 \\
\hline
\end{tabular}

$\mathrm{NA}=-30, \mathrm{TAA}=10$

\section{D30: Nuts for Coolant Plate, F281-283}

3 Nuts

\begin{tabular}{|l|l|l|}
\hline Criteria & Comments & Scoring \\
\hline N1 & 7 movements for each nut $* 3=21$ & 1 \\
\hline N2 & Assume 5s for each nut $=15 \mathrm{~s}$ & 0 \\
\hline N3 & HV protection needed & 1 \\
\hline N4 & Low weight, some bending & -1 \\
\hline N5 & $\begin{array}{l}\text { Necessary to reach Cells/ Modules, coolant plate itself is also a lot of recyclable } \\
\text { metal }\end{array}$ & 2 \\
\hline T1 & Standard unscrewing & 2 \\
\hline T2 & Open access for end-effector & 2 \\
\hline T3 & Same color as Coolant plate, but easy to detect nut from top & 1 \\
\hline T4 & Some choices & 1 \\
\hline T5 & Simple handling of screws, sorting into metal collection & 2 \\
\hline
\end{tabular}

$\mathrm{NA}=30, \mathrm{TAA}=80$ 


\section{D31: Coolant Plate Section 5, P45}

1 large coolant plate

\begin{tabular}{|l|l|l|}
\hline Criteria & Comments & Scoring \\
\hline N1 & Move, grasp, bring, release, two hands = & -1 \\
\hline N2 & Assume 30s & 0 \\
\hline N3 & HV danger and possibly danger due to coolant & 2 \\
\hline N4 & Assume 5-10kg of weight, some bending necessary & 0 \\
\hline N5 & Necessary for Cells / Modules, also the large portion of recyclable metal & 2 \\
\hline T1 & More complex grabbing/ lifting operation & 0 \\
\hline T2 & Open access from top, but tool must be specialized to grab it from side & 1 \\
\hline T3 & Good detection of large part, more difficult to find spot to grab & 1 \\
\hline T4 & Some choices, unsure how good working for that size & 0 \\
\hline T5 & Large part liquid (coolant) involved & -1 \\
\hline
\end{tabular}

$\mathrm{NA}=30, \mathrm{TAA}=10$

\section{D32: Insulating Pad Battery Section 5, P46}

1 large insulating pad

\begin{tabular}{|l|l|l|}
\hline Criteria & Comments & Scoring \\
\hline N1 & Move, grasp, bring, release +2 for loosening below $=6$ & -1 \\
\hline N2 & Assume 20s & 0 \\
\hline N3 & HV danger & 1 \\
\hline N4 & Low weight, some bending & -1 \\
\hline N5 & Necessary to reach Cells/ Modules, low-cost itself & 1 \\
\hline T1 & More complicated grabbing due to glued connection below & 0 \\
\hline T2 & $\begin{array}{l}\text { Good access from top, but more difficult to get below, maybe just strong } \\
\text { grabbing }\end{array}$ & 1 \\
\hline T3 & Good to detect from the top, more difficult to find glued spot below & 1 \\
\hline T4 & Grabber, but unsure if it would be strong enough & 0 \\
\hline T5 & Larger but light part, collecting for reuse or put in larger trash & 0 \\
\hline
\end{tabular}

$\mathrm{NA}=0, \mathrm{TAA}=20$ 


\section{D33: Cover Battery Section 4, P47}

1 large Cover

\begin{tabular}{|l|l|l|}
\hline Criteria & Comments & Scoring \\
\hline N1 & Move, grasp, bring, release, 2 hands =8 & -1 \\
\hline N2 & Assume 30s & 1 \\
\hline N3 & HV danger & 1 \\
\hline N4 & Assume 5-10kg, some bending & -1 \\
\hline N5 & Necessary to reach Cells/ Modules and large part of metal to recycle & 2 \\
\hline T1 & Grabbing of large part & 0 \\
\hline T2 & Open access from top, grabbing from side & 1 \\
\hline T3 & Easy to detect from top, more complicated to detect spot to grab at side & 1 \\
\hline T4 & Some choices, but larger part & 0 \\
\hline T5 & Handling of large part, collecting large metal parts for further recycling & 1 \\
\hline
\end{tabular}

$\mathrm{NA}=20, \mathrm{TAA}=30$

D34: Clips HV Harness Battery Section 4, F327-328

2 Clips

\begin{tabular}{|l|l|l|}
\hline Criteria & Comments & Scoring \\
\hline N1 & 3 motions for unplugging *2 $=6$ & -1 \\
\hline N2 & Assume 20s & 0 \\
\hline N3 & HV danger & 1 \\
\hline N4 & Low weights, some bending & -1 \\
\hline N5 & Necessary to reach Cells/ Modules, but low-cost & 1 \\
\hline T1 & More complex unplugging or cutting & -1 \\
\hline T2 & Open access, but size limitations & 1 \\
\hline T3 & Partly hidden, bad contrast, very small sizes & -1 \\
\hline T4 & Some concepts for cutting but unsure if working & -1 \\
\hline T5 & No parts, only unplugging & 0 \\
\hline
\end{tabular}

$\mathrm{NA}=0, \mathrm{TAA}=-10$ 
D35: Side Brackets Battery Section 4, P48-49

2 Brackets

\begin{tabular}{|l|l|l|}
\hline Criteria & Comments & Scoring \\
\hline N1 & 4 movements for grabbing $* 2=8$ & -1 \\
\hline N2 & Assume 20s & 0 \\
\hline N3 & HV protection needed & 1 \\
\hline N4 & Low weight, some bending is necessary & -1 \\
\hline N5 & Necessary to reach Cells/ Modules & 1 \\
\hline T1 & Simple grabbing operation (medium size part) & 2 \\
\hline T2 & Very good access from side & 1 \\
\hline T3 & $\begin{array}{l}\text { Detection could be a little difficult because of same color, but position and } \\
\text { shape should be recognizable }\end{array}$ & 1 \\
\hline T4 & Some choices for grabber, should work on this & 1 \\
\hline T5 & Medium size part, placing into metal collection & 1 \\
\hline
\end{tabular}

$\mathrm{NA}=0, \mathrm{TAA}=60$

\section{D36: Bolts Battery Section 4, F337-342}

6 Bolts

\begin{tabular}{|l|l|l|}
\hline Criteria & Comments & Scoring \\
\hline N1 & 7 movements for each bolt $* 6=42$ & 2 \\
\hline N2 & Assume 5s for each bolt $6=30$ & 1 \\
\hline N3 & HV danger & 1 \\
\hline N4 & Low weight, but some bending & -1 \\
\hline N5 & Necessary to reach Cells/ Modules & 1 \\
\hline T1 & Simple unscrewing & 2 \\
\hline T2 & Open access from the top, some size limitations & 1 \\
\hline T3 & Good detection from top possible & 2 \\
\hline T4 & Some choices for automated screwdriver & 1 \\
\hline T5 & Simple collection of metal fasteners & 1 \\
\hline
\end{tabular}

$\mathrm{NA}=40, \mathrm{TAA}=70$ 
D37: Retainers, Position Assurance Battery Section 4, F329-336

4 Retainer Clips, 4 Position Insurance Clips

\begin{tabular}{|l|l|l|}
\hline Criteria & Comments & Scoring \\
\hline N1 & Move, position, cut * 8=24 & 1 \\
\hline N2 & Assume 5s for each unclipping *8=40 & 1 \\
\hline N3 & HV danger & 1 \\
\hline N4 & Low weight, some bending & -1 \\
\hline N5 & Necessary to reach Cells/ Modules, but low-cost & 1 \\
\hline T1 & More complex cutting or strong pushing & -1 \\
\hline T2 & Open access from Top/Side & 1 \\
\hline T3 & Good detection from Top/Side & 1 \\
\hline T4 & Not sure about choices & -1 \\
\hline T5 & No parts & 0 \\
\hline
\end{tabular}

$\mathrm{NA}=30, \mathrm{TAA}=0$

\section{D38: HV Monitoring Circuit Battery Section 4, P55-56}

2 Sense Lines (cables)

\begin{tabular}{|l|l|l|}
\hline Criteria & Comments & Scoring \\
\hline N1 & 4 movements for grabbing $=8$ & -1 \\
\hline N2 & Assume 20s & 0 \\
\hline N3 & HV protection needed & 1 \\
\hline N4 & Low weight, some bending/twisting & -1 \\
\hline N5 & Necessary to reach cells, but not valuable itself & 1 \\
\hline T1 & Grabbing of cables & 1 \\
\hline T2 & Some size limitations for grabber & 1 \\
\hline T3 & Good detectable, different shape and color compared to surrounding parts & 2 \\
\hline T4 & Some choices for grabber of cables, small parts & 1 \\
\hline T5 & Small part, sorting into trash or collection of cables & 1 \\
\hline
\end{tabular}

$\mathrm{NA}=0, \mathrm{TAA}=60$ 
D39: Battery Sections 1-4 Lifting, P36, P39, P50, P57

4 Battery Sections

\begin{tabular}{|l|l|l|}
\hline Criteria & Comments & Scoring \\
\hline N1 & Move, position, adjust, move, use lifting tool, move, release *4=32 & 2 \\
\hline N2 & Assume 90s each =360s & 2 \\
\hline N3 & HV danger, if damaged possibly chemical danger & 2 \\
\hline N4 & Very high weight, about 60-70kg & 2 \\
\hline N5 & Modules/ Sections & 1 \\
\hline T1 & More complicated lifting operation & 0 \\
\hline T2 & Side access needed, open & 1 \\
\hline T3 & Easy to detect Modules/Sections, but more difficult to find spot at sides & 1 \\
\hline T4 & Some choices & 1 \\
\hline T5 & Large part to handle, place at different station for further disassembly & 0 \\
\hline
\end{tabular}

$\mathrm{NA}=90, \mathrm{TAA}=30$

D40: Heat Transfer Mats, P63-70

8 Heat Transfer Mats

\begin{tabular}{|l|l|l|}
\hline Criteria & Comments & Scoring \\
\hline N1 & Normally 4 motions for grabbing +1because of glue $* 8=40$ & 2 \\
\hline N2 & Assume 50s & 1 \\
\hline N3 & Only sharp edges possible & -1 \\
\hline N4 & Low weight, some bending, twisting for ungluing it & 0 \\
\hline N5 & Not valuable itself, just material sorting & -1 \\
\hline T1 & More complex grabbing & 0 \\
\hline T2 & Open access from top, but more difficult to get below mat & 0 \\
\hline T3 & Easy to detect because of shape and color & 2 \\
\hline T4 & Some choices, but unsure if those would work on that special case & -1 \\
\hline T5 & Sorting mats into trash (probably not reusable), maybe problems due to glue & 0 \\
\hline
\end{tabular}

$\mathrm{NA}=10, \mathrm{TAA}=10$ 
D41: Bolts for Braces, Coolant Plate, F343-356, F361-371, F375

26 Bolts

\begin{tabular}{|l|l|l|}
\hline Criteria & Comments & Scoring \\
\hline N1 & 7 movements each $=182$ & 2 \\
\hline N2 & Assume 5s each $=130$ s & 2 \\
\hline N3 & Only sharp edges & -1 \\
\hline N4 & Low weight of fasteners, some bending & -1 \\
\hline N5 & Only sorting of materials & -1 \\
\hline T1 & Just simple bolts, no tool changing necessary & 2 \\
\hline T2 & Open access from the top & 2 \\
\hline T3 & Detection from the top, different color, but not that good to detect & 1 \\
\hline T4 & Some choices: e.g. Li et al. [26] & 1 \\
\hline T5 & Collection of Bolts, sorting in Screw/Bolt/ Nut collection & 2 \\
\hline
\end{tabular}

$\mathrm{NA}=10, \mathrm{TAA}=80$

\section{D42: Braces, P58-61}

4 Braces

\begin{tabular}{|l|l|l|}
\hline Criteria & Comments & Scoring \\
\hline N1 & 4 movements each for grabbing *4=16 & 0 \\
\hline N2 & Assume 5s each = 20s & 0 \\
\hline N3 & Only sharp edges & -1 \\
\hline N4 & Low weight, assume 1-5kg each, some bending & -1 \\
\hline N5 & Only material sorting & -1 \\
\hline T1 & Grabbing of medium size part & 1 \\
\hline T2 & Open access from top & 2 \\
\hline T3 & Good detection of braces & 2 \\
\hline T4 & Some choices, should work on braces & 1 \\
\hline T5 & Handling of medium/large parts, sorting into metal trash & 1 \\
\hline
\end{tabular}

$\mathrm{NA}=-30, \mathrm{TAA}=70$ 
D43: Remaining Bolts for Coolant Plate, F372-374

3 Bolts

\begin{tabular}{|l|l|l|}
\hline Criteria & Comments & Scoring \\
\hline N1 & 7 movements each $* 3=21$ & 1 \\
\hline N2 & Assume 5s each $=15 \mathrm{~s}$ & 0 \\
\hline N3 & Only sharp edges & -1 \\
\hline N4 & Low weight of fasteners, some bending & -1 \\
\hline N5 & Only sorting of materials & -1 \\
\hline T1 & Just simple bolts, no tool changing necessary & 2 \\
\hline T2 & Open access from the top & 2 \\
\hline T3 & Detection from the top, different color, but not that good to detect & 1 \\
\hline T4 & Some choices: e.g. Li et al. [26] & 1 \\
\hline T5 & Collection of Bolts, sorting in Screw/Bolt/ Nut collection & 2 \\
\hline
\end{tabular}

$\mathrm{NA}=-20, \mathrm{TAA}=80$

\section{D44: Coolant Plate, P71}

1 large coolant plate

\begin{tabular}{|l|l|l|}
\hline Criteria & Comments & Scoring \\
\hline N1 & Move, grasp, bring, release, two hands $=8$ & -1 \\
\hline N2 & Assume 30s & 0 \\
\hline N3 & Sharp edges or danger due to coolant & 0 \\
\hline N4 & $\begin{array}{l}\text { Assume 10-15kg of weight, some bending necessary, far away from body due } \\
\text { to size }\end{array}$ & 1 \\
\hline N5 & Only material sorting but large metal part itself to recycle & 0 \\
\hline T1 & More complex grabbing/ lifting operation & 0 \\
\hline T2 & Open access from top, but tool must be specialized to grab it from side & 1 \\
\hline T3 & Good detection of large part, more difficult to find spot to grab & 1 \\
\hline T4 & Some choices, unsure how good working for that size & 0 \\
\hline T5 & Large part liquid (coolant) involved & -1 \\
\hline
\end{tabular}

$\mathrm{NA}=0, \mathrm{TAA}=10$ 
D45: Insulating Pads, P72-75

4 Insulating Pads

\begin{tabular}{|l|l|l|}
\hline Criteria & Comments & Scoring \\
\hline N1 & Move, grasp, bring, release +2 for loosening below $* 4=24$ & 1 \\
\hline N2 & Assume 60s & 2 \\
\hline N3 & No dangers & -2 \\
\hline N4 & Low weight, some bending & -1 \\
\hline N5 & Only material sorting & -1 \\
\hline T1 & More complicated grabbing due to glued connection below & 0 \\
\hline T2 & $\begin{array}{l}\text { Good access from top, but more difficult to get below, maybe just strong } \\
\text { grabbing }\end{array}$ & 1 \\
\hline T3 & Good to detect from the top, more difficult to find glued spot below & 1 \\
\hline T4 & Grabber, but unsure if it would be strong enough & 0 \\
\hline T5 & Larger but light part, collecting for reuse or put in larger trash & 0 \\
\hline
\end{tabular}

$\mathrm{NA}=-10, \mathrm{TAA}=20$

\section{D46: Handling of Battery Tray, P76}

1 Large Tray

\begin{tabular}{|l|l|l|}
\hline Criteria & Comments & Scoring \\
\hline N1 & $\begin{array}{l}\text { At least two persons: Move, position, adjust, move, use lifting tool*2, move, } \\
\text { release }=16\end{array}$ & 0 \\
\hline N2 & Assume $90 \mathrm{~s}$ & 2 \\
\hline N3 & Only sharp edges possible & -1 \\
\hline N4 & Very high weight, assume $>25 \mathrm{~kg}$ & 2 \\
\hline N5 & Has to be taken somewhere, large part of metal for further recycling & 1 \\
\hline T1 & More complicated lifting operation & 0 \\
\hline T2 & Side access needed, open & 1 \\
\hline T3 & Easy to detect Tray, could be grabbed somewhere at side & 1 \\
\hline T4 & Some choices & 1 \\
\hline T5 & Large part to handle, place at collection for large metal parts & 1 \\
\hline
\end{tabular}

$\mathrm{NA}=40, \mathrm{TAA}=40$ 


\subsubsection{Overview of Assessments for HEV Battery}

Assessment in TAA and NA for all 15 disassembly steps down to the level of battery modules and 4 more steps for the disassembly of the battery modules of the Audi Q5

hybrid battery disassembly can be seen in the following table:

\begin{tabular}{|l|l|l|}
\hline Step \# & NA & TAA \\
\hline Step I: Unscrew covers and casing bottom: & 30 & 20 \\
\hline $\begin{array}{l}\text { Step II: Removal of power electronics cover and side } \\
\text { covering: }\end{array}$ & -10 & 50 \\
\hline $\begin{array}{l}\text { Step III: Disassembly of the live lines from the } \\
\text { modules/ stacks: }\end{array}$ & $?$ & $?$ \\
\hline Step IV: Cutting of cable ties: & 10 & 20 \\
\hline $\begin{array}{l}\text { Step V: Disassembly of the plug connection between } \\
\text { the cell controllers and the BMS: }\end{array}$ & -10 & -10 \\
\hline Step VI: Removal of BMS and power electronics: & 20 & 30 \\
\hline Step VII: Cutting of the bus for the thermo sensors: & -10 & 0 \\
\hline Step VIII: Disassembly and removal of system cover: & -20 & 50 \\
\hline Step IX: Unscrew and removing of cable guiding: & 40 & 50 \\
\hline $\begin{array}{l}\text { Step X: Removal of gas venting and the cover of the } \\
\text { stacks: }\end{array}$ & 20 & 60 \\
\hline $\begin{array}{l}\text { Step XI: Disassembly and removal of the connector } \\
\text { between the stacks: }\end{array}$ & 30 & 0 \\
\hline Step XII: Unscrew and removal of stack holders: & 50 & 60 \\
\hline Step XIII: Removal of casing bottom: & -20 & 30 \\
\hline Step XIV: Unscrew and removal of stack fastener: & 40 & 60 \\
\hline Step XV: Removal of stacks: & 70 & 30 \\
\hline Steps for the disassembly of the battery modules: & 40 & 00 \\
\hline Step I: Unscrewing of nuts on the cell contacts & 40 & 0 \\
\hline Step II: Removal of cables and cell connectors & 40 & 30 \\
\hline Step III: Unscrewing and removal of the side covers: & 30 & \\
\hline Step IV: Removal of battery cells: & 60 & \\
\hline
\end{tabular}




\subsubsection{Detailed Assessments of each Disassembly step for HEV Battery}

\section{Step 1 (I): Unscrew covers and casing bottom:}

15-20 bolts, nuts, from top, sides, bottom

\begin{tabular}{|l|l|l|}
\hline Criteria & Comments & Scoring \\
\hline N1 & $\begin{array}{l}\text { Move, position, unscrew, move, grasp, bring, release for every } \\
\text { screw/bolt/nut + tool change ca. } 7 * 20=140\end{array}$ & 2 \\
\hline N2 & $\begin{array}{l}\text { 5s for every screw/nut, approx. 120s in total with screwdriver } \\
\text { grabbing/ tool change }\end{array}$ & 2 \\
\hline N3 & $\begin{array}{l}\text { No high voltage or chemical dangers at that point, only sharp } \\
\text { edges possible }\end{array}$ & -1 \\
\hline N4 & $\begin{array}{l}\text { Very low weights, just screws/bolts and nuts, some bending to } \\
\text { reach screws }\end{array}$ & -1 \\
\hline N5 & $\begin{array}{l}\text { Necessary to reach cells because no other possibilities to come to } \\
\text { cells/modules }\end{array}$ & 2 \\
\hline T1 & Tool changing seems necessary & 1 \\
\hline T2 & Access form sides and bottom needed, more difficult, but open & -1 \\
\hline T3 & Also, detection on sides and bottom needed & 0 \\
\hline T4 & Some choices: e.g. Li et al. [26] & 1 \\
\hline T5 & Just collection of screws/nuts/bolts & 2 \\
\hline
\end{tabular}

$\mathrm{NA}=40, \mathrm{TAA}=30$

\section{Step 2 (II): Removal of power electronics cover and side covering:}

2 covers, grabber needed

\begin{tabular}{|l|l|l|}
\hline Criteria & Comments & Scoring \\
\hline N1 & Move, grasp, bring, release $* 2$ & -1 \\
\hline N2 & Ca.20s for all steps & 0 \\
\hline N3 & Only sharp edges possible & -1 \\
\hline N4 & Approx. 0.5 kg for each cover, but bending & -1 \\
\hline N5 & $\begin{array}{l}\text { High priority, because the covers are the first step to reach the } \\
\text { cells/modules }\end{array}$ & 2 \\
\hline T1 & Translational movement and grabbing of larger part & 0 \\
\hline T2 & Open access to covers, but grabbing from side & 1 \\
\hline T3 & Detection from side needed, more need for tactile robot & 1 \\
\hline T4 & Some choices: e.g. Weigl-Seitz et al. [28] & 1 \\
\hline T5 & Metal covers can be collected or just put into metal trash & 2 \\
\hline
\end{tabular}

$\mathrm{NA}=-10, \mathrm{TAA}=50$ 


\section{Step 3 (III): Disassembly of the live lines from the modules/ stacks:}

Loosening of the screws for the electrical contacts

\begin{tabular}{|l|l|l|}
\hline Criteria & Comments & Scoring \\
\hline N1 & Assume 12 motions & 0 \\
\hline N2 & Assume 20s & 0 \\
\hline N3 & HV danger & 1 \\
\hline N4 & Some bending, but low weight & -1 \\
\hline N5 & High priority but not valuable itself & 1 \\
\hline T1 & More complicated unscrewing operation & 0 \\
\hline T2 & Open access & 2 \\
\hline T3 & Detection more difficult & 0 \\
\hline T4 & Some screwdriver choices & 1 \\
\hline T5 & Fastener collecting & 2 \\
\hline
\end{tabular}

$\mathrm{NA}=10, \mathrm{TAA}=50$

\section{Step 4 (IV): Cutting of cable ties:}

One cable tie (many cables inside), cutting operation, maybe cut 2 times to get it out.

\begin{tabular}{|l|l|l|}
\hline Criteria & Comments & Scoring \\
\hline N1 & $\begin{array}{l}\text { Only a few motions, because just one operation, grab cutter, } \\
\text { move to cable ties, cut cable tie, move away, release cutter (not } \\
\text { sure, if there are much more connections to get cut) }\end{array}$ & -1 \\
\hline N2 & Ca. 20s & 0 \\
\hline N3 & High voltage protection needed & 1 \\
\hline N4 & Low weight, less than 1kg, but bending & -1 \\
\hline N5 & Cables are connected to modules (also needed for other parts) & 2 \\
\hline T1 & More complex, cutting & 0 \\
\hline T2 & $\begin{array}{l}\text { Open access from top, but size limitations and difficult because it } \\
\text { moves }\end{array}$ & 0 \\
\hline T3 & Open from top, but cables are more difficult to detect & 1 \\
\hline T4 & $\begin{array}{l}\text { Some choices, but not sure how good fitting: e.g. Bailey-Van } \\
\text { Kuren [32] }\end{array}$ & -1 \\
\hline T5 & $\begin{array}{l}\text { Only cables, not special treatment (either cable collection or } \\
\text { residual trash) }\end{array}$ & 2 \\
\hline
\end{tabular}

NA: 10, TAA: 20 


\section{Step 5 (V): Disassembly of the plug connection between the cell controllers and the}

BMS:

Cutting or unplugging needed

\begin{tabular}{|l|l|l|}
\hline Criteria & Comments & Scoring \\
\hline N1 & Low number & -1 \\
\hline N2 & Medium amount of time & 0 \\
\hline N3 & High voltage danger & 1 \\
\hline N4 & Low weight, bending & -1 \\
\hline N5 & Needed to reach cells and BMS & 2 \\
\hline T1 & $\begin{array}{l}\text { Very complex (if unplugging), or more complex (if cutting), } \\
\text { assume cutting }\end{array}$ & 0 \\
\hline T2 & Size limitations but open access (from side) & -1 \\
\hline T3 & Side view, partly hidden & -1 \\
\hline T4 & $\begin{array}{l}\text { Some choices, but not sure how good fitting: e.g. Bailey-Van } \\
\text { Kuren [32] }\end{array}$ & -1 \\
\hline T5 & No parts to remove & 2 \\
\hline
\end{tabular}

NA: -10 , TAA: -10

\section{Step 6 (VI): Removal of BMS and power electronics:}

Assume $2 * 4$ screws/bolts, different ones

\begin{tabular}{|l|l|l|}
\hline Criteria & Comments & Scoring \\
\hline N1 & 7 movements for each screw/bolt and removal of parts: $>60$ & 2 \\
\hline N2 & Ca. 30s for unscrewing and removal & 1 \\
\hline N3 & High voltage protection needed & 1 \\
\hline N4 & Low weight, some bending to side of battery & -1 \\
\hline N5 & $\begin{array}{l}\text { Not sure if necessary, to reach cells, but parts itself could be } \\
\text { valuable }\end{array}$ & 1 \\
\hline T1 & Unscrewing task with bit changing & 1 \\
\hline T2 & Side access but open & 1 \\
\hline T3 & Side detection, bad contrast, small screws & 0 \\
\hline T4 & (see above), choices for screws/ bolts & 1 \\
\hline T5 & $\begin{array}{l}\text { Screw/bolt collection, but special collection of BMS and Power } \\
\text { Electronics }\end{array}$ & 0 \\
\hline
\end{tabular}

NA: 20 , TAA: 30 


\section{Step 7 (VII): Cutting of the bus for the thermo sensors:}

Side cutter, cutting tool, not sure, how many cutting operations (assume 1)

\begin{tabular}{|l|l|l|}
\hline Criteria & Comments & Scoring \\
\hline N1 & Low number & -1 \\
\hline N2 & Assume ca. 10s, should be fast & -1 \\
\hline N3 & High voltage protection needed & 1 \\
\hline N4 & Low weight, but bending to reach bus & -1 \\
\hline N5 & Should be connected to battery, so necessary for modules & 1 \\
\hline T1 & Cutting operation & 0 \\
\hline T2 & Not sure, assume more difficult access (at least less space) & 0 \\
\hline T3 & Assume that sensors are difficult to detect & -1 \\
\hline T4 & $\begin{array}{l}\text { Some choices, but not sure how good fitting: e.g. Bailey-Van } \\
\text { Kuren [32] }\end{array}$ & -1 \\
\hline T5 & Residual trash (if sensors are removed with that step) & 2 \\
\hline
\end{tabular}

NA: -10 , TAA: 0

\section{Step 8 (VIII): Disassembly and removal of system cover:}

Unscrewing has been done in I, just removal

\begin{tabular}{|l|l|l|}
\hline Criteria & Comments & Scoring \\
\hline N1 & Move, grasp, bring, release & -1 \\
\hline N2 & Ca.12s for all movements & -1 \\
\hline N3 & Only sharp edges possible & -1 \\
\hline N4 & Approx. 0.5 kg for each cover, but little bending for body & -1 \\
\hline N5 & $\begin{array}{l}\text { High priority, because the covers are the first step to reach the } \\
\text { cells/modules }\end{array}$ & 2 \\
\hline T1 & Translational movement and grabbing of larger part & 0 \\
\hline T2 & Open access to covers, but grabbing from side & 1 \\
\hline T3 & Detection from side needed, more fore needed, tactile robot & 1 \\
\hline T4 & Some choices: e.g. Bòrras et al. [83] & 1 \\
\hline T5 & Metal covers can be collected or just put into metal trash & 2 \\
\hline
\end{tabular}

NA: -20, TAA: 50 


\section{Step 9 (IX): Unscrew and removing of cable guiding:}

Assume 4 screws (top), one cable guiding

\begin{tabular}{|l|l|l|}
\hline Criteria & Comments & Scoring \\
\hline N1 & 7 motions $x$ 4 screws, removal of the guiding $>32$ & 2 \\
\hline N2 & Assume ca. 30s & 1 \\
\hline N3 & High voltage protection needed & 1 \\
\hline N4 & Low weight, but little bending & -1 \\
\hline N5 & $\begin{array}{l}\text { Not sure, if needed for modules, assume yes, but no cable } \\
\text { guiding has no high value }\end{array}$ & 1 \\
\hline T1 & Simple movements, assume one type of screw & 2 \\
\hline T2 & Limited size for end effector, but access from top & 1 \\
\hline T3 & $\begin{array}{l}\text { Not sure about detection, but should be open with some } \\
\text { problems }\end{array}$ & 1 \\
\hline T4 & Some choices, unscrewing, grabbing & 1 \\
\hline T5 & Two different kinds of materials & 0 \\
\hline
\end{tabular}

NA: 40, TAA: 50

\section{Step 10 (X): Removal of gas venting and the cover of the stacks:}

Assume 4 covers and one gas venting

\begin{tabular}{|l|l|l|}
\hline Criteria & Comments & Scoring \\
\hline N1 & 4 movements for grabbing * 5 & 0 \\
\hline N2 & Assume 30s for removing all parts & 1 \\
\hline N3 & High voltage protection needed & 1 \\
\hline N4 & Low weight, but little bending of body & -1 \\
\hline N5 & Necessary to reach Modules, not valuable itself & 1 \\
\hline T1 & Grabbing movements & 2 \\
\hline T2 & Open access from top & 2 \\
\hline T3 & Difficult to differentiate because of same color to nearby parts & 1 \\
\hline T4 & Grabbing tool, some choices & 1 \\
\hline T5 & More than one type of material, larger size & 0 \\
\hline
\end{tabular}

NA: 20, TAA: 60 
Step 11 (XI): Disassembly and removal of the connector between the stacks:

Unplugging/ cutting

\begin{tabular}{|l|l|l|}
\hline Criteria & Comments & Scoring \\
\hline N1 & Some connections, assume quite many motions & 1 \\
\hline N2 & Assume ca. 30s & 1 \\
\hline N3 & High voltage protection needed & 1 \\
\hline N4 & Low weight, but bending needed & -1 \\
\hline N5 & Necessary to get to modules & 1 \\
\hline T1 & More complex, assume cutting, or prying & 0 \\
\hline T2 & Size limitations, maybe partly hidden & 0 \\
\hline T3 & More difficult to detect, no color difference, small part & -1 \\
\hline T4 & Choice for cutting, but unsure & -1 \\
\hline T5 & Should be just some connectors for residual trash & 2 \\
\hline
\end{tabular}

NA: 30 , TAA: 0 , not sure, could be worse in both

\section{Step 12 (XII): Unscrew and removal of stack holders:}

Can only describe unscrewing: Assume $>10$ screws

\begin{tabular}{|l|l|l|}
\hline Criteria & Comments & Scoring \\
\hline N1 & Many movements & 2 \\
\hline N2 & Longer time & 2 \\
\hline N3 & High voltage & 1 \\
\hline N4 & Low weight, but bending & -1 \\
\hline N5 & Necessary to reach modules, cheap itself & 1 \\
\hline T1 & Simple screws & 2 \\
\hline T2 & Open access (not completely sure, maybe size) & 1 \\
\hline T3 & Maybe somehow hidden & 1 \\
\hline T4 & Some existing choices (unscrewing, grabbing) & 1 \\
\hline T5 & Screw handling easy but stack holders & 1 \\
\hline
\end{tabular}

NA: 50, TAA: 60 
Step 13 (XIII): Removal of casing bottom:

Grabbing operation

\begin{tabular}{|l|l|l|}
\hline Criteria & Comments & Scoring \\
\hline N1 & Move, grasp, bring, release & -1 \\
\hline N2 & Ca.10s for all movements & -1 \\
\hline N3 & Only sharp edges possible & -1 \\
\hline N4 & Approx. 0.5 kg for each cover, but bending & -1 \\
\hline N5 & $\begin{array}{l}\text { iigh priority, because the covers are the first step to reach the } \\
\text { cells/modules }\end{array}$ & 2 \\
\hline T1 & Translational movement and grabbing of larger part & 0 \\
\hline T2 & Open access to cover, but grabbing from bottom & 0 \\
\hline T3 & Detection from bottom needed, more need fore tactile robot & 0 \\
\hline T4 & Some choices & 1 \\
\hline T5 & Metal covers can be collected or just put into metal trash & 2 \\
\hline
\end{tabular}

NA: -20, TAA: 30

\section{Step 14 (XIV): Unscrew and removal of stack fastener:}

6 Screws on picture

\begin{tabular}{|l|l|l|}
\hline Criteria & Comments & Scoring \\
\hline N1 & $7 * 6$ movements, + access to screwdriver and removing of part & 2 \\
\hline N2 & Assume ca. 30s & 1 \\
\hline N3 & High voltage protection needed & 1 \\
\hline N4 & Low weight, but some bending & -1 \\
\hline N5 & High priority for modules, not valuable itself & 1 \\
\hline T1 & Unscrewing operation and grabbing operation & 2 \\
\hline T2 & Open access from top & 2 \\
\hline T3 & Screws got different color; they are normal size & 2 \\
\hline T4 & Some choices: Unscrewing, grabbing & 1 \\
\hline T5 & Simple collection of screws, stack fastener is larger & 1 \\
\hline
\end{tabular}

NA: 40 , TAA: 80 


\section{Step 15 (XV): Removal of stacks:}

Large stacks (modules)

\begin{tabular}{|l|l|l|}
\hline Criteria & Comments & Scoring \\
\hline N1 & 2 people needed: approach, grasp, lift, bring, place, 4 stacks & 2 \\
\hline N2 & Assume ca. 2min & 2 \\
\hline N3 & High high-voltage danger, & 1 \\
\hline N4 & $\begin{array}{l}\text { Assume 7kg for each stack, but also bending, maybe a little bit } \\
\text { more far away from body }\end{array}$ & 0 \\
\hline N5 & Highest priority because it is the module removal & 2 \\
\hline T1 & More complex movements, lifting tool & 0 \\
\hline T2 & More difficult to grab, maybe extended (and strong) grabber & 1 \\
\hline T3 & More difficult to detect place to grab (maybe partly hidden) & 1 \\
\hline T4 & Some choices: e.g. Bòrras et al. [83] & 1 \\
\hline T5 & Collection of large recycled part & 1 \\
\hline
\end{tabular}

NA: 70, TAA: 40 


\section{Steps for modules of Audi Q5 Hybrid:}

\section{Step 16 (I): Unscrewing of nuts on the cell contacts}

18 nuts

\begin{tabular}{|l|l|l|}
\hline Criteria & Comments & Scoring \\
\hline N1 & 18 nuts & 2 \\
\hline N2 & Longer time for all nuts & 2 \\
\hline N3 & High voltage protection needed & 1 \\
\hline N4 & $\begin{array}{l}\text { Low weight, only screws, also not bending, it the smaller module } \\
\text { is disassembled in and optimized workspace }\end{array}$ & -2 \\
\hline N5 & High priority to reach cells & 1 \\
\hline T1 & Simple unscrewing & 2 \\
\hline T2 & Open Access from top & 2 \\
\hline T3 & Open view and different color & 2 \\
\hline T4 & Some choices & 1 \\
\hline T5 & Collecting of nuts & 2 \\
\hline
\end{tabular}

NA: 40, TAA: 90

\section{Step 17 (II): Removal of cables and cell connectors}

Grabbing and unplugging, 8 cell connectors

\begin{tabular}{|l|l|l|}
\hline Criteria & Comments & Scoring \\
\hline N1 & Unplugging of 8 connectors, at least 3 motions for unplugging & 2 \\
\hline N2 & $\begin{array}{l}\text { Would take longer than one minute (depends of difficulty of } \\
\text { unplugging) }\end{array}$ & 2 \\
\hline N3 & High voltage danger & 1 \\
\hline N4 & Low weight, some bending for difficult unplugging & -1 \\
\hline N5 & High priority to reach cells & 1 \\
\hline T1 & Very complex, unplugging & -2 \\
\hline T2 & Open access from top (cutting and unplugging) & 2 \\
\hline T3 & Open view from top (cutting and unplugging) & 2 \\
\hline T4 & No idea of a proposed concept for that unplugging & -2 \\
\hline T5 & Handling of cables & 0 \\
\hline
\end{tabular}

NA: 40, TAA: 0 
Step 18 (III): Unscrewing and removal of the side covers:

Assume 4 screws, 2 covers

\begin{tabular}{|l|l|l|}
\hline Criteria & Comments & Scoring \\
\hline N1 & 8 Screws and grabbing of two covers & 2 \\
\hline N2 & Assume ca. 30s & 1 \\
\hline N3 & High voltage protection needed & 1 \\
\hline N4 & Low weight, but bending or twisting because of side access & -1 \\
\hline N5 & High priority to reach cells & 1 \\
\hline T1 & Simple screws, rotational and translational movements for robot & 2 \\
\hline T2 & Open access, but side & 1 \\
\hline T3 & Open view, but side & 1 \\
\hline T4 & Some choices for screwdrivers & 1 \\
\hline T5 & Screw collection, but covers are a little larger & 1 \\
\hline
\end{tabular}

NA: 30, TAA: 60

\section{Step 19 (IV): Removal of battery cells:}

18 cells

\begin{tabular}{|c|c|c|}
\hline Criteria & Comments & Scoring \\
\hline N1 & 18 cells, at least 4 movements for each cell & 2 \\
\hline $\mathrm{N} 2$ & Assume 2 minutes & 2 \\
\hline N3 & High voltage danger, and chemical, if on cell is broken & 2 \\
\hline N4 & Low weight (approx. $0.25-0.5 \mathrm{~kg}$ ) but bending to reach those & -1 \\
\hline N5 & High priority, cells are the valuable part & 1 \\
\hline $\mathrm{T} 1$ & Grabbing motion & 2 \\
\hline $\mathrm{T} 2$ & Not sure about access, but access from top & 1 \\
\hline T3 & $\begin{array}{l}\text { Not sure about detection of place to grab, vision system can see } \\
\text { place of symmetric placed cells }\end{array}$ & 1 \\
\hline $\mathrm{T} 4$ & At least one Choice: Schmitt et al. [63] & 0 \\
\hline T5 & $\begin{array}{l}\text { Larger size, some special treatment needed, dangerous materials } \\
\text { inside }\end{array}$ & -1 \\
\hline
\end{tabular}

NA: 60, TAA: 30 


\subsection{Parameters for Simulation}

\section{Parts for Battery:}

\begin{tabular}{|l|l|l|l|l|}
\hline Part or Fastener & Quantity & $\begin{array}{l}\text { Part in real } \\
\text { EV battery }\end{array}$ & $\begin{array}{l}\text { Size: } \\
\text { length, width, height [mm }\end{array}$ & $\begin{array}{l}\text { Location: x, y, z } \\
\text { (works process) }\end{array}$ \\
\hline Battery Tray & 1 & P81 & $1600 \times 1000 \times 150$ & $0,0,350$ \\
\hline $\begin{array}{l}\text { Top Cover } \\
\text { (upper part) }\end{array}$ & 1 & P1 & $\begin{array}{l}1000 \times 1600 \times 20 \\
(400,920,130)\end{array}$ & $0,0,500$ \\
\hline $\begin{array}{l}\text { Bolts for Top } \\
\text { Cover } \\
\text { (left/ right, top } \\
\text { left/ top right) }\end{array}$ & 26 & F1-56 & $18 \times 20.8 \times 10$ & $-/+480,-780,520 ;$ \\
\hline $\begin{array}{l}\text { Bolts for Electrical } \\
\text { Connector } \\
\text { Housing }\end{array}$ & 4 & F357-360 & $21 \times 24 \times 10$ & $-200,500,650 ;$ \\
\hline $\begin{array}{l}\text { Electrical } \\
\text { Connector } \\
\text { Housing }\end{array}$ & 1 & P62 & $30 \times 100 \times 100$ & $-320,-830,-410$ \\
\hline $\begin{array}{l}\text { Cable } \\
\text { Bolts for Brackets } \\
\text { (left / right) }\end{array}$ & 4 & e.g. 248 & $18 \times 20.8 \times 10$ & $-300,-800,430$ \\
\hline $\begin{array}{l}\text { Brackets } \\
\text { (upper part) }\end{array}$ & 4 & e.g. P32 & $\begin{array}{l}20 \times 200 \times 100 \\
(60 \times 200 \times 20)\end{array}$ & $-330 / 110,-680,370$ \\
\hline $\begin{array}{l}\text { Battery Sections } \\
\text { (Modules) }\end{array}$ & 3 & e.g. P36 & $300 \times 900 \times 100$ & $0,-500,370$ \\
\hline
\end{tabular}




\section{Robots, Conveyors and Tools: Gantry robot layout}

\begin{tabular}{|l|l|l|l|l|l|}
\hline Tool & $\begin{array}{l}\text { Quan } \\
\text {-tity }\end{array}$ & $\begin{array}{l}\text { Real Battery } \\
\text { Disassembly }\end{array}$ & $\begin{array}{l}\text { Size: } \\
{[\mathrm{mm}]}\end{array}$ & $\begin{array}{l}\text { Location: } \\
\mathrm{x}, \mathrm{y}, \mathrm{z}\end{array}$ & More Data \\
\hline $\begin{array}{l}\text { Cartesian } \\
\text { Gantry robot }\end{array}$ & 1 & Cartesian robot & $\begin{array}{l}2700 \times 3000 \mathrm{x} \\
1800\end{array}$ & $-1100,1500,0$ & $\begin{array}{l}\text { Cartesian speed: } \\
2000 \mathrm{~mm} / \mathrm{s}\end{array}$ \\
\hline $\begin{array}{l}\text { Generic } \\
\text { Vacuum } \\
\text { Gripper 1 }\end{array}$ & 1 & $\begin{array}{l}\text { Screwdriver/ } \\
\text { Nutrunner }\end{array}$ & $\begin{array}{l}\text { Cup diameter: } 30 \\
\text { Cup offset: } 140\end{array}$ & $1300,1400,840$ & \\
\hline $\begin{array}{l}\text { Generic } \\
\text { Vacuum } \\
\text { Gripper 2 }\end{array}$ & 1 & $\begin{array}{l}\text { Screwdriver/ } \\
\text { Nutrunner }\end{array}$ & $\begin{array}{l}\text { Cup diameter: } 40 \\
\text { Cup offset: } 140\end{array}$ & $1300,1200,840$ & \\
\hline $\begin{array}{l}\text { Suction } \\
\text { Gripper 1 }\end{array}$ & 1 & Large grabbing tool & $\begin{array}{l}75 \times 75 \times 50 \\
\text { Suction cups: } \\
5 \times 5\end{array}$ & $1600,1400,830$ & \\
\hline $\begin{array}{l}\text { Suction } \\
\text { Gripper 2 }\end{array}$ & 1 & $\begin{array}{l}\text { Medium/ Small } \\
\text { grabbing tool }\end{array}$ & $\begin{array}{l}30 \times 30 \times 50 \\
\text { Suction cups: } \\
5 \times 5\end{array}$ & $1600,1100,830$ & \\
\hline $\begin{array}{l}\text { Works } \\
\text { process } \\
\begin{array}{l}\text { Conveyor } \\
\text { left }\end{array}\end{array}$ & 1 & Fastener Handling & $500 \times 500 \times 10$ & $-1000,300,500$ & $\begin{array}{l}\text { Conveyor } \\
\text { speed: } \\
1000 \mathrm{~mm} / \mathrm{s}\end{array}$ \\
\hline $\begin{array}{l}\text { Works } \\
\text { process } \\
\text { Conveyor } \\
\text { right }\end{array}$ & 1 & Parts Handling & $800,1800,10$ & $1600, .200,400$ & $\begin{array}{l}\text { Conveyor } \\
\text { speed: } \\
1000 \mathrm{~mm} / \mathrm{s}\end{array}$ \\
\hline $\begin{array}{l}\text { Working } \\
\text { table }\end{array}$ & 1 & Working table & $\begin{array}{l}1600 \times 1000 \mathrm{x} \\
35 \beta\end{array}$ & $0,0,0$ & \\
\hline
\end{tabular}




\section{Robots, Conveyors and Tools: Collaborative robots layout}

\begin{tabular}{|c|c|c|c|c|c|}
\hline Tool & $\begin{array}{l}\text { Quan } \\
\text {-tity }\end{array}$ & $\begin{array}{l}\text { Real Battery } \\
\text { Disassembly }\end{array}$ & $\begin{array}{l}\text { Size: } \\
{[\mathrm{mm}]}\end{array}$ & $\begin{array}{l}\text { Location: } \\
\mathrm{x}, \mathrm{y}, \mathrm{z}\end{array}$ & More Data \\
\hline UR10e robot & 2 & UR10e robot & $\begin{array}{l}-/+970,1200 \\
400\end{array}$ & $-/+970,1200,400$ & $\begin{array}{l}\text { Cartesian speed: } \\
200 \mathrm{~mm} / \mathrm{s}\end{array}$ \\
\hline $\begin{array}{l}\text { Generic } \\
\text { Vacuum } \\
\text { Gripper } 1\end{array}$ & 2 & $\begin{array}{l}\text { Screwdriver/ } \\
\text { Nutrunner }\end{array}$ & $\begin{array}{l}\text { Cup diameter: } \\
30 \\
\text { Cup offset: } 140\end{array}$ & $-/+1800,600,820$ & \\
\hline $\begin{array}{l}\text { Generic } \\
\text { Vacuum } \\
\text { Gripper } 2\end{array}$ & 1 & $\begin{array}{l}\text { Screwdriver/ } \\
\text { Nutrunner }\end{array}$ & $\begin{array}{l}\text { Cup diameter: } \\
40 \\
\text { Cup offset: } 140\end{array}$ & $-/+1800,800,820$ & \\
\hline $\begin{array}{l}\text { Suction } \\
\text { Gripper } 2\end{array}$ & 1 & $\begin{array}{l}\text { Medium/ } \\
\text { Small } \\
\text { grabbing tool }\end{array}$ & $\begin{array}{l}30 \times 30 \times 50 \\
\text { Suction cups: } \\
5 \times 5\end{array}$ & $1600,1100,830$ & \\
\hline $\begin{array}{l}\text { Works process } \\
\text { Conveyor } \\
\text { left }\end{array}$ & 1 & $\begin{array}{l}\text { Fastener } \\
\text { Handling }\end{array}$ & $500 \times 500 \times 10$ & $-1650,-1200,500$ & \\
\hline $\begin{array}{l}\text { Works process } \\
\text { Conveyor } \\
\text { right }\end{array}$ & 1 & Parts Handling & $500 \times 500 \times 10$ & $1650.1200,500$ & \\
\hline Working table & 1 & Working table & $\begin{array}{l}1600 \times 1000 \times \\
35 \beta\end{array}$ & $0,0,0$ & \\
\hline
\end{tabular}




\section{Bibliography}

[1] R. Hannappel, "The impact of global warming on the automotive industr," 2017, vol. 1871, no. 060001, pp. 1-7.

[2] C. Thies, K. Kieckhäfer, C. Hoyer, and T. S. Spengler, "Economic Assessment of the LithoRec Process," in Recycling of Lithium-Ion Batteries, 2018, pp. 253-266.

[3] N. Wu, Jimmy, Mackkenzie, Andrew, Scharma, "Recycling lithium-ion batteries: adding value with multiple lives," Green Chem., vol. 22, no. 7, pp. 2244-2254, 2020 .

[4] D. J. Garole, R. Hossain, V. J. Garole, V. Sahajwalla, J. Nerkar, and D. Dubal, "Recycle, Recover and Repurpose Strategy of Spent Li-ion Batteries and Catalysts: Current Status and Future Opportunities," ChemSusChem, vol. 13, pp. 1-23, 2020.

[5] J. Diekmann, S. Rothermel, S. Nowak, and A. Kwade, "The LithoRec Process," in Recycling of Lithium-Ion Batteries, 2018, pp. 33-38.

[6] K. Wegener, W. H. Chen, F. Dietrich, K. Dröder, and S. Kara, "Robot assisted disassembly for the recycling of electric vehicle batteries," in Procedia CIRP, 2015, vol. 29, pp. 716-721.

[7] B. Ketterer, U. Karl, D. Möst, and S. Ulrich, "Lithium-Ionen Batterien: Stand der Technik und Anwendungspotenzial in Hybrid-, Plug-In Hybrid- und Elektrofahrzeugen," 2009.

[8] N. Weyrich, M, Natkunarajah, "Konzeption einer automatischen Demontageanlage für Lithium-Ionen-Batterien," in VDI Automatisierungskongress, 2013.

[9] Green Car Congress, "First drive: US spec Audi A3 Sportback e-tron plug-in hybrid; 83-86 MPGe with 16-17 mile EV range," 2015. [Online]. Available: https://www.greencarcongress.com/2015/11/20151103-a3.html.

[10] K. Wegener, "Mensch-Roboter-Kooperation zur Demontage von Traktionsbatterien," Ph.D dissertation, Technische Universität Braunschweig, 2015.

[11] G. Harper et al., "Recycling lithium-ion batteries from electric vehicles," Nature, vol. 575, no. 7781, pp. 75-86, 2019.

[12] A. Tornow, S. Andrew, F. Dietrich, and K. Dröder, "Impact of multi-material components on the assembly and disassembly of traction batteries," in Procedia CIRP, 2015, vol. 29, pp. 792-797.

[13] M. Elwert, Tobias, Römer, Felix, Schneider, Kirstin, Hua, Qingsong, Buchert, "Recycling of Batteries from Electric Vehicles," in Behaviour of Lithium-Ion Batteries in Electric Vehicles, 2018, pp. 289-321. 
[14] S.-W. Choi, Yong, Rhee, "Current status and perspectives on recycling of end-oflife battery of electric vehicle in Korea (Republic of)," Waste Manag., vol. 106, pp. 261-270, 2020.

[15] Z. Liu, X. Liu, H. Hao, F. Zhao, A. A. Amer, and H. Babiker, "Research on the critical issues for power battery reusing of new energy vehicles in China," Energies, vol. 13, no. 8, pp. 1-19, 2020.

[16] L. Gaines, "Profitable Recycling of Low-Cobalt Lithium-Ion Batteries Will Depend on New Process Developments," One Earth, vol. 1, no. 4, pp. 413-415, 2019.

[17] L. Gaines, "Lithium-ion battery recycling processes: Research towards a sustainable course," Sustain. Mater. Technol., vol. 17, no. e00068, pp. 1-7, 2018.

[18] L. Ahmadi, S. B. Young, M. Fowler, R. A. Fraser, and M. A. Achachlouei, “A cascaded life cycle: reuse of electric vehicle lithium-ion battery packs in energy storage systems," Int. J. Life Cycle Assess., vol. 22, no. 1, pp. 111-124, 2017.

[19] E. Mossali, N. Picone, L. Gentilini, O. Rodrìguez, J. M. Pérez, and M. Colledani, "Lithium-ion batteries towards circular economy: A literature review of opportunities and issues of recycling treatments," J. Environ. Manage., vol. 264, pp. 1-12, 2020.

[20] D. Werner, U. A. Peuker, and T. Mütze, "Recycling chain for spent lithium-ion batteries," Metals (Basel)., vol. 10, no. 3, 2020.

[21] J. D. Kelly, "2017 Chevrolet Bolt EV Battery Disassembly," 2017 Chevrolet Bolt EV Battery Disassembly, 2018. [Online]. Available: https://www.youtube.com/watch?v=ssU2mjiNi_Q.

[22] J. D. Kelly, "2017 Chevrolet Bolt EV Battery Reassembly,” 2018. [Online]. Available: https://www.youtube.com/watch?v=ZBzRKglr95U\&t=6050.

[23] W. D. Li, K. Xia, L. Gao, and K. M. Chao, "Selective disassembly planning for waste electrical and electronic equipment with case studies on liquid crystal displays," Robot. Comput. Integr. Manuf., vol. 29, no. 4, pp. 248-260, 2013.

[24] H. Wang, Q. Peng, J. Zhang, and P. Gu, "Selective disassembly planning for the end-of-life product," in Procedia CIRP, 2017, vol. 60, pp. 512-517.

[25] M. Nave, "Beitrag zur automatisierten Demontage durch Optimierung des Trennprozesses von Schraubenverbindungen," Ph.D dissertation, Universität Dortmund, 2003.

[26] R. Li et al., "Unfastening of Hexagonal Headed Screws by a Collaborative Robot," IEEE Trans. Autom. Sci. Eng., pp. 1-14, 2020.

[27] M. Bdiwi, A. Rashid, M. Pfeifer, and M. Putz, "Disassembly of unknown models of electrical vehicle motors using innovative human robot cooperation," HRI '17: Proceedings of the Companion of the 2017 ACM/IEEE International Conference on Human-Robot. pp. 85-86, 2017. 
[28] A. Weigl-Seitz, K. Hohm, M. Seitz, and H. Tolle, "On strategies and solutions for automated disassembly of electronic devices," Int. J. Adv. Manuf. Technol., vol. 30, no. 5-6, pp. 561-573, 2006.

[29] A. Weigl, "Exemplarische Untersuchungen zur flexiblen automatisierten Demontage elektronischer Geräte mit Industrierobotern," Ph.D dissertation, RWTH Aachen, 1997.

[30] A. Stenzel, "Beitrag zum flexiblen Greifen in der Alexander Stenzel Beitrag zum flexiblen Greifen in der Demontage," Technischen Universität Berlin, 2001.

[31] P. Schumacher and M. Jouaneh, "A force sensing tool for disassembly operations," Robot. Comput. Integr. Manuf., vol. 30, no. 2, pp. 206-217, 2014.

[32] M. Bailey-Van Kuren, "Flexible robotic demanufacturing using real time tool path generation," Robot. Comput. Integr. Manuf., vol. 22, no. 1, pp. 17-24, 2006.

[33] B. Scholz-Reiter, H. Scharke, and A. Hucht, "Flexible robot-based disassembly cell for obsolete TV-sets and monitors," Robot. Comput. Integr. Manuf., vol. 15, no. 3, pp. 247-255, 1999.

[34] E. Kroll and T. A. Hanft, "Quantitative evaluation of product disassembly for recycling," Res. Eng. Des. - Theory, Appl. Concurr. Eng., vol. 10, no. 1, pp. 1-14, 1998.

[35] M. Kerin and D. T. Pham, "A review of emerging industry 4.0 technologies in remanufacturing," J. Clean. Prod., vol. 237, pp. 1-16, 2019.

[36] J. Li, M. Barwood, and S. Rahimifard, "Robotic disassembly for increased recovery of strategically important materials from electrical vehicles," Robot. Comput. Integr. Manuf., vol. 50, no. March 2016, pp. 203-212, 2018.

[37] S. Smith, L. Y. Hsu, and G. C. Smith, "Partial disassembly sequence planning based on cost-benefit analysis," J. Clean. Prod., vol. 139, pp. 729-739, 2016.

[38] S. Vongbunyong, S. Kara, and M. Pagnucco, "Learning and revision in cognitive robotics disassembly automation," Robot. Comput. Integr. Manuf., vol. 34, pp. 7994, 2015.

[39] E. Kroll, "Application of work-measurement analysis to product diassembly for recycling," Concurr. Eng. Res. Appl., vol. 4, no. 2, pp. 149-158, 1996.

[40] U. Rebafka, G. Seliger, A. Stenzel, and B. R. Zuo, "Process model based development of disassembly tools," Proc. Inst. Mech. Eng. Part B J. Eng. Manuf., vol. 215, no. 5, pp. 711-721, 2001.

[41] K. Feldmann, S. Trautner, and O. Meedt, "Innovative Disassembly Strategies Based on Flexible Partial Destructive Tools," IFAC Proc. Vol., vol. 31, no. 7, pp. $1-6,1998$.

[42] B. R. Zuo, A. Stenzel, and G. Seliger, "A novel disassembly tool with screwnail endeffectors,” J. Intell. Manuf., vol. 13, no. 3, pp. 157-163, 2002. 
[43] G. Seliger, T. Keil, U. Rebafka, and A. Stenzel, "Flexible disassembly tools," IEEE Int. Symp. Electron. Environ., pp. 30-35, 2001.

[44] J. R. Peeters, P. Vanegas, C. Mouton, W. Dewulf, and J. R. Duflou, "Tool Design for Electronic Product Dismantling," Procedia CIRP, vol. 48, pp. 466-471, 2016.

[45] G. Tian, M. Zhou, and J. Chu, "A chance constrained programming approach to determine the optimal disassembly sequence," IEEE Trans. Autom. Sci. Eng., vol. 10, no. 4, pp. 1004-1013, 2013.

[46] H. Hohm, K., Müller Hofstede, H., Tolle, "Robot assisted Disassembly of Elecotronic Devices," in Proceedings. 2000 EEE/RSJ International Conference on Intelligent Robots and Systems (IROS 2000), 2000, pp. 1273-1278.

[47] H. Kim, R. Harms, and G. Seliger, "Automatic Control Sequence Generation for a Hybrid Disassembly System," IEEE Trans. Autom. Sci. Eng., vol. 4, no. 2, pp. 194 205, 2007.

[48] N. DiFilippo, "Framework for the automated disassembly of electronic waste using the Soar cognitive architecture," Ph.D dissertation, University of Rhode Island, 2016.

[49] K. M. Lee and M. M. Bailey-Van Kuren, "Modeling and supervisory control of a disassembly automation workcell based on blocking topology," IEEE Trans. Robot. Autom., vol. 16, no. 1, pp. 67-77, 2000.

[50] Y. Tang, M. C. Zhou, E. Zussman, and R. Caudill, "Disassembly modeling, planning, and application," J. Manuf. Syst., vol. 21, no. 3, pp. 200-217, 2002.

[51] Y. Tang and M. Zhou, "A Systematic Approach to Design and Operation of Disassembly Lines," in Short Papers, 2006, vol. 3, no. 3, pp. 324-329.

[52] N. M. Difilippo and M. K. Jouaneh, "A System Combining Force and Vision Sensing for Automated Screw Removal on Laptops," IEEE Trans. Autom. Sci. Eng., vol. 15, no. 2, pp. 887-895, 2018.

[53] P. Gil, J. Pomares, S. V. T. Puente, C. Diaz, F. Candelas, and F. Torres, "Flexible multi-sensorial system for automatic disassembly using cooperative robots," Int. $J$. Comput. Integr. Manuf., vol. 20, no. 8, pp. 757-772, 2007.

[54] F. Torres, P. Gil, S. T. Puente, J. Pomares, and R. Aracil, "Automatic PC disassembly for component recovery," Int. J. Adv. Manuf. Technol., vol. 23, no. 12, pp. 39-46, 2004.

[55] H. Poschmann, H. Brüggemann, and D. Goldmann, "Disassembly 4.0: A Review on Using Robotics in Disassembly Tasks as a Way of Automation," ChemieIngenieur-Technik, vol. 92, no. 4, pp. 341-359, 2020.

[56] A. C. Honem de Mello, Luiz S., Sanderson, "And/or graph representation of assembly plans," IEEE Trans. Robot. Autom., vol. 6, no. 2, pp. 188-199, 1990. 
[57] T. Cao and A. C. Sanderson, "AND/OR Net Representation for Robotic Task Sequence Planning," IEEE Trans. Syst. Man Cybern. Part C Appl. Rev., vol. 28, no. 2, pp. 204-218, 1998.

[58] K. Wegener, S. Andrew, A. Raatz, K. Dröder, and C. Herrmann, "Disassembly of electric vehicle batteries using the example of the Audi Q5 hybrid system," in Procedia CIRP, 2014, vol. 23, pp. 155-160.

[59] F. Cerdas et al., "Disassembly Planning and Assessment of Automation Potentials for Lithium-Ion Batteries," in Recycling of Lithium-Ion Batteries, 2018, pp. 83-97.

[60] C. Herrmann, A. Raatz, M. Mennenga, J. Schmitt, and S. Andrew, "Assessment of automation potentials for the disassembly of automotive lithium ion battery systems," in Leveraging Technology for a Sustainable World - Proceedings of the 19th CIRP Conference on Life Cycle Engineering, 2012, pp. 149-154.

[61] J. Li, M. Barwood, and S. Rahimifard, "A multi-criteria assessment of robotic disassembly to support recycling and recovery," Resour. Conserv. Recycl., vol. 140, no. September 2018, pp. 158-165, 2019.

[62] T. E. Schwarz, W. Rübenbauer, B. Rutrecht, and R. Pomberger, "Forecasting Real Disassembly Time of Industrial Batteries Based on Virtual MTM-UAS Data," in Procedia CIRP, 2018, vol. 69, pp. 927-931.

[63] J. Schmitt, H. Haupt, M. Kurrat, and A. Raatz, "Disassembly automation for lithium-ion battery systems using a flexible gripper," in IEEE 15th International Conference on Advanced Robotics: New Boundaries for Robotics, ICAR 2011, 2011, pp. 291-297.

[64] L. Boothroyd, G. , Alting, "Design for Assembly and Disassembly," CIRP Ann., vol. 41, no. 2, pp. 625-636, 1992.

[65] M. I. Campbell and A. Hasan, "Design evaluation method for the disassembly of electronic equipment," Proc. Int. Conf. Eng. Des. ICED, vol. DS 31, pp. 1-10, 2003.

[66] H. Chen and J. Shen, "A degradation-based sorting method for lithium-ion battery reuse," PLoS One, vol. 12, no. 10, pp. 1-15, 2017.

[67] S. Maharshi and K. Janardhan Reddy, "Cloud based disassembly of electric vehicle battery," in Procedia Manufacturing, 2019, vol. 30, pp. 136-142.

[68] M. Kampker, Achim, Heimes, Heiner, Lienemann, Christoph, Grauel, Daniel, Jones, "Development of a novel remanufacturing architecture for lithium-ion battery packs," in 2017 Electric Vehicles International Conference (EV), Bucharest, 2017, pp. 1-6.

[69] S. Kay, Ian, Esmaeeli, Roja, Hashemi, Seyed Reza, Mahajan, Ajay, Farhad, "Recycling Li-Ion Batteries: Robotic Disassembly of Electric Vehicle Battery Systems," in Proceedings of the ASME 2019 International Mechanical Engineering Congress and Exposition. Volume 6: Energy. Salt Lake City, Utah, USA., 2019, pp. $1-10$. 
[70] M. A. Goodrich and A. C. Schultz, "Human-robot interaction: A survey," Found. Trends Human-Computer Interact., vol. 1, no. 3, pp. 203-275, 2007.

[71] A. Murata, "Ergonomics and cognitive engineering for robot-human cooperation," in Proceedings - IEEE International Workshop on Robot and Human Interactive Communication, 2000, pp. 206-211.

[72] E. Lotter, "Hybride Montagesysteme," in Montage in der industriellen Produktion: Ein Handbuch für die Praxis. 2., Lotter, Bruno, Wiendahl, Hans-Peter, 2012, pp. 167-193.

[73] B. D. Argall, S. Chernova, M. Veloso, and B. Browning, "A survey of robot learning from demonstration," Rob. Auton. Syst., vol. 57, no. 5, pp. 469-483, 2009.

[74] T. Gecks, "Sensorbasierte, echtzeitfähige Online-Bahnplanung für die MenschRoboter-Koexistenz," Ph.D dissertation, Universität Bayreuth, 2011.

[75] A. De Luca, A. Albu-Schäffer, S. Haddadin, and G. Hirzinger, "Collision detection and safe reaction with the DLR-III lightweight manipulator arm," in IEEE International Conference on Intelligent Robots and Systems, 2006, pp. 1623-1630.

[76] S. Vongbunyong, P. Vongseela, and J. Sreerattana-Aporn, "A Process Demonstration Platform for Product Disassembly Skills Transfer," Procedia CIRP, vol. 61, no. c, pp. 281-286, 2017.

[77] J. Zhang, H. Liu, Q. Chang, L. Wang, and R. X. Gao, "Recurrent neural network for motion trajectory prediction in human-robot collaborative assembly," in CIRP Annals - Manufacturing Technology, 2020, vol. 00, pp. 8-11.

[78] W. H. Chen, K. Wegener, and F. Dietrich, "A robot assistant for unscrewing in hybrid human-robot disassembly," in 2014 IEEE International Conference on Robotics and Biomimetics, IEEE ROBIO 2014, 2014, pp. 536-541.

[79] E. Yildiz and F. Worgotter, "DCNN-Based Screw Detection for Automated Disassembly Processes," in 15th International Conference on Signal-Image Technology \& Internet-Based Systems (SITIS) DCNN-Based, 2019, pp. 187-192.

[80] Z. Zazar Gandler, C. H. Ek, M. Björkman, R. Stolkin, and Y. Bekiroglu, “Object shape estimation and modeling, based on sparse Gaussian process implicit surfaces , combining visual data and tactile exploration," Rob. Auton. Syst., vol. 126, no. 103433, pp. 1-16, 2020.

[81] C. B. Kristensen, F. A. Sørensen, H. B. Nielsen, M. S. Andersen, S. P. Bendtsen, and S. Bøgh, "Towards a robot simulation framework for E-waste disassembly using reinforcement learning," Procedia Manuf., vol. 38, no. 2019, pp. 225-232, 2019.

[82] W. Paetsch, "Exemplarische Untersuchungen zu mehrfingrigen Robotergreifern: Aufbau - Regelung - Systemintegration,” VDI-Reihe 8, Nr 363, Düsseldorf, 1993. 
[83] J. Borras, R. Heudorfer, S. Rader, P. Kaiser, and T. Asfour, “The KIT Swiss Knife Gripper for Disassembly Tasks: A Multi-Functional Gripper for Bimanual Manipulation with a Single Arm," in IEEE International Conference on Intelligent Robots and Systems, 2018, pp. 4590-4597.

[84] M. Adjigble, N. Marturi, V. Ortenzi, V. Rajasekaran, P. Corke, and R. Stolkin, "Model-free and learning-free grasping by Local Contact Moment matching," in IEEE International Conference on Intelligent Robots and Systems, 2018, pp. 29332940.

[85] N. Marturi et al., "Dynamic grasp and trajectory planning for moving objects," Auton. Robots, vol. 43, no. 5, pp. 1241-1256, 2019.

[86] R. Gerbers, K. Wegener, F. Dietrich, and K. Dröder, "Safe, Flexible and Productive Human-Robot-Collaboration for Disassembly of Lithium-Ion Batteries," in Recycling of Lithium-Ion Batteries, 2018, pp. 99-126.

[87] M. Ortenzi, V., Stolkin, R., Kuo, J. \& Mistry, "Hybrid motion/force control: a review," Acvanced Robot., vol. 31, no. 19-20, pp. 1102-1113, 2017.

[88] C. Hoyer, Strategische Planung des Recyclings von Lithium-Ionen-Batterien aus Elektrofahrzeugen in Deutschland. 2015.

[89] R. Gerbers, M. Mücke, F. Dietrich, and K. Dröder, "Simplifying Robot Tools by Taking Advantage of Sensor Integration in Human Collaboration Robots," in Procedia CIRP, 2016, vol. 44, pp. 287-292.

[90] J. R. Duflou, G. Seliger, S. Kara, Y. Umeda, A. Ometto, and B. Willems, "Efficiency and feasibility of product disassembly: A case-based study," CIRP Ann. - Manuf. Technol., vol. 57, no. 2, pp. 583-600, 2008.

[91] K. Mahmood and F. Gutteridge, "EV Batteries Remanufacturing," , BORG automotive challenge, University of Strathclyde, 2019.

[92] A. Kampker, H. Heimes, M. Ordung, C. Lienemann, A. Hollah, and N. Sarovic, "Evaluation of a Remanufacturing for Lithium Ion Batteries from Electric Cars," Int. J. Mech. Mechatronics Eng., vol. 10, no. 12, pp. 1922-1928, 2016.

[93] I. Mathews, B. Xu, W. He, V. Barreto, T. Buonassisi, and I. M. Peters, "Technoeconomic model of a second-life energy storage system for utility-scale solar power considering li-ion calendar and cycle aging," Preprint, 2020.

[94] L. Cong, F. Zhao, and J. W. Sutherland, "Integration of dismantling operations into a value recovery plan for circular economy," J. Clean. Prod., vol. 149, pp. 378$386,2017$.

[95] C. White, B. Thompson, and L. G. Swan, "Repurposed electric vehicle battery performance in second-life electricity grid frequency regulation service," J. Energy Storage, vol. 28, no. February, p. 101278, 2020.

[96] K. B. Zadin, Most: Work Measurement Systems. Pittsburgh, Pennsylvania: H. B. Maynard and Company, Inc., 2003. 
[97] U. Steinberg and H.-J. Windberg, "Heben und Tragen ohne Schaden," Bundesanstalt für Arbeitsschutz und Arbeitsmedizin (BAuA). pp. 1-18, 2011.

[98] Universal Robots, "THE UR10e COLLABORATIVE INDUSTRIAL ROBOT." [Online]. Available: https://www.universal-robots.com/products/ur10-robot/. 